\title{
Two-dimensional pnictogens: A review of recent progresses and future research directions 8
}

\author{
Cite as: Appl. Phys. Rev. 6, 021308 (2019); doi: 10.1063/1.5074087 \\ Submitted: 21 October 2018 . Accepted: 8 February 2019 . \\ Published Online: 22 April 2019
}
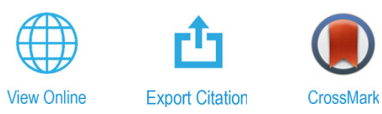

\author{
F. Ersan, ${ }^{1,2}$ (D) D. Kecik, ${ }^{2,3}$ (D) V. O. Özçelik, ${ }^{4}$ Y. Kadioglu, ${ }^{1,2}$ O. Üzengi Aktürk, ${ }^{5,6}$ E. Durgun, ${ }^{3, a)}$ (D) E. Aktürk, ${ }^{1,5, b)}$ (DD \\ and S. Ciraci ${ }^{2, c)}$
}

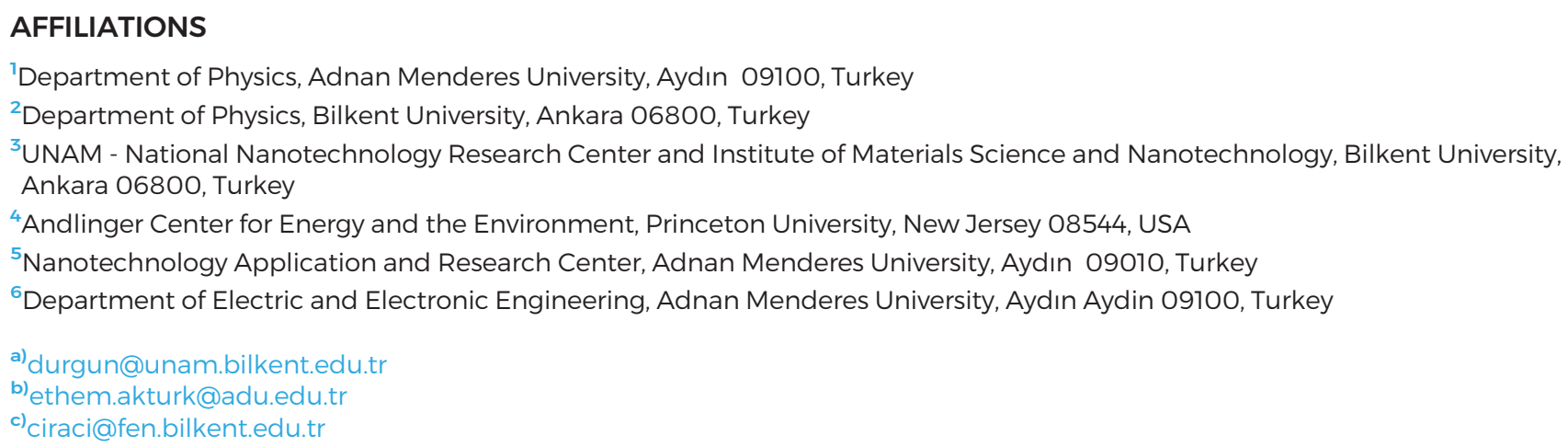

\begin{abstract}
Soon after the synthesis of two-dimensional (2D) ultrathin black phosphorus and fabrication of field effect transistors thereof, theoretical studies have predicted that other group-VA elements (or pnictogens), N, As, Sb, and Bi can also form stable, single-layer (SL) structures. These were nitrogene in a buckled honeycomb structure, arsenene, antimonene, and bismuthene in a buckled honeycomb, as well as washboard and square-octagon structures with unusual mechanical, electronic, and optical properties. Subsequently, theoretical studies are followed by experimental efforts that aim at synthesizing these novel 2D materials. Currently, research on $2 \mathrm{D}$ pnictogens has been a rapidly growing field revealing exciting properties, which offers diverse applications in flexible electronics, spintronics, thermoelectrics, and sensors. This review presents an evaluation of the previous experimental and theoretical studies until 2019, in order to provide input for further research attempts in this field. To this end, we first reviewed 2D, SL structures of group-VA elements predicted by theoretical studies with an emphasis placed on their dynamical and thermal stabilities, which are crucial for their use in a device. The mechanical, electronic, magnetic, and optical properties of the stable structures and their nanoribbons are analyzed by examining the effect of external factors, such as strain, electric field, and substrates. The effect of vacancy defects and functionalization by chemical doping through adatom adsorption on the fundamental properties of pnictogens has been a critical subject. Interlayer interactions in bilayer and multilayer structures, their stability, and tuning their physical properties by vertical stacking geometries are also discussed. Finally, our review is concluded by highlighting new research directions and future perspectives on the challenges in this emerging field.
\end{abstract}

Published under license by AIP Publishing. https://doi.org/10.1063/1.5074087

\section{TABLE OF CONTENTS}

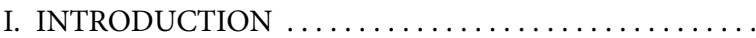

II. ATOMIC STRUCTURE, ENERGETICS, STABILITY,

AND THERMAL PROPERTIES . . . . . . . . . . . .

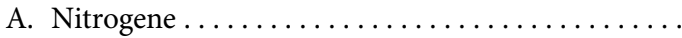

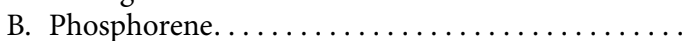

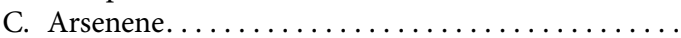

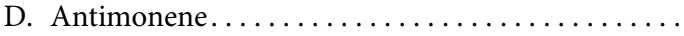

E. Bismuthene. .................................
III. ELECTRONIC AND OPTICAL PROPERTIES. . . . . . .

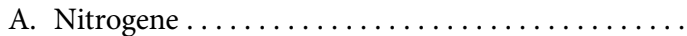

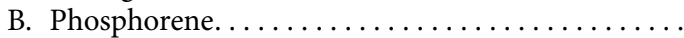

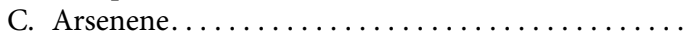

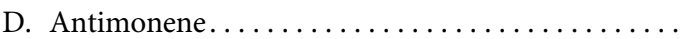

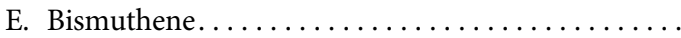

IV. ADATOMS, ADMOLECULES, AND DEFECTS:

FUNCTIONALIZATION $\ldots \ldots \ldots \ldots \ldots \ldots \ldots \ldots$

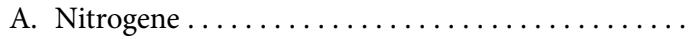




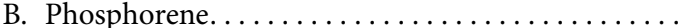

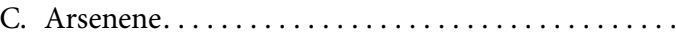

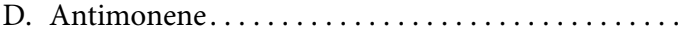

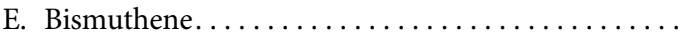

F. Hydrogenation and halogenation $\ldots \ldots \ldots \ldots \ldots$

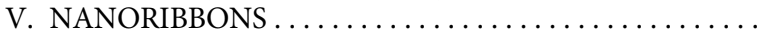

VI. BILAYER AND MULTILAYERS. . . . . . . . . . .

VII. SUBSTRATE EFFECTS . . . . . . . . . . . . . . .

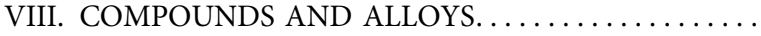

IX. EXPERIMENTAL RESULTS $\ldots \ldots \ldots \ldots \ldots \ldots \ldots$

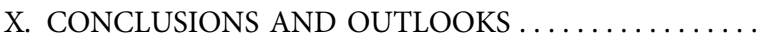

12

13

13

13

16

16

17

17

\section{INTRODUCTION}

During the last decade, there has been growing interest in twodimensional (2D) materials due to their unique chemical, mechanical, electronic, and optical properties. ${ }^{1-6}$ Motivated by the synthesis of graphene and its extraordinary properties, ${ }^{4-6}$ researchers have focused on predicting and synthesizing $2 \mathrm{D}$ allotropes of other elements and compounds. On the theoretical front, these efforts led to the prediction of 2D allotropes of group-IV elements, group IV, III-V, and II-VI compounds, ${ }^{7-15}$ silicon dioxides, ${ }^{16}$ transition metal dioxides ${ }^{17,18}$ and dichalcogenides, ${ }^{17,19-22}$ monochalcogenides, ${ }^{23-25}$ and alkali-earth metal hydroxides. ${ }^{26,27}$ It was shown that these $2 \mathrm{D}$ materials display diverse electronic, optical, and magnetic properties for a wide range of technological applications, such as optoelectronics, spintronics, catalysts, chemical and biological sensors, supercapacitors, solar cells, nanotribology, ${ }^{28-30}$ hydrogen production, and lithium or sodium ion batteries. $^{31,32}$

Very recently, the synthesis of ultrathin, 2D black phosphorus, through exfoliation from its layered bulk counterparts, has brought the suspended 2D monolayers and multilayers of group-VA elements ${ }^{33-35}$ into the focus of current research. Specifically, the field effect transistors (FETs) fabricated on few-layer black phosphorus ${ }^{33}$ showed a high field-effect mobility up to $1000 \mathrm{~cm}^{2} / \mathrm{V}$ s at room temperature for a thickness of $\sim 10 \mathrm{~nm}{ }^{33}$ Additionally, on the basis of first-principles calculations, blue phospherene, also named as buckled honeycomb phospherene, was predicted to be a stable allotrope. ${ }^{34}$ As the growing research on phosphorene unveiled interesting properties, such as anisotropy, layer-dependent photoluminescence (PL) and quasi $1 \mathrm{D}$ excitations, ${ }^{33,35-43}$ the quest for predicting similar $2 \mathrm{D}$ allotropes of other group-VA elements attracted the interest of many researchers.

Only after two years from the synthesis of black phosphorene, theoretical studies have predicted stable, monolayer structures and thin films of group-VA elements, such as N, As, Sb, and Bi. Seminal results are summarized in Fig. 1. These materials were named as nitrogene ${ }^{44}$ in the hexagonal buckled $(h b)$ structure, arsenene in $h b$ - and symmetric washboard $(s w)$ structures, ${ }^{45}$ and antimonene ${ }^{46,47}$ and bismuthene ${ }^{48}$ in either $h b$ - or asymmetric washboard (aw) structures. Later, phosphorene, arsenene, antimonene, and bismuthene were predicted to form also stable square-octagon (so) structures, ${ }^{49}$ as well as other exotic structures. Their stabilities at room temperature were justified by calculating their phonon frequencies and performing high temperature molecular dynamics (MD) simulations. ${ }^{44,46,48,50,51}$ In contrast to semimetallic monolayers of group-IV elements, monolayers of group-VA elements are stable semiconductors with bandgaps suitable for several device applications, which have been the driving force for numerous theoretical and experimental studies. Motivated by these growing research activities, this paper presents a concise review of the most recent studies on 2D monolayer and multilayer pnictogens and also an outline of future prospects and emerging challenges in theory and applications. Our objective is to draw attention to seminal theoretical and experimental studies with the hope of shedding light on future research. Previous papers on phosphorene $e^{52-60}$ and other pnictogens ${ }^{5,61-64}$ are complemented by placing emphasis on the most recent achievements. This review predominantly focuses and presents exhaustive results on: (i) the most recent progress in $2 \mathrm{D}$ pnictogen research, (ii) features which have not been reviewed before, and (iii) provides a very extensive and comprehensive literature survey on $2 \mathrm{D}$ pnictogens.

\section{ATOMIC STRUCTURE, ENERGETICS, STABILITY, AND THERMAL PROPERTIES}

While nitrogene appears only in the $h b$ form, ${ }^{44}$ monolayers of other pnictogens can form stable $h b$-, sw- or $a w$-, and so-structures. ${ }^{34,45-49}$ In contrast to $s w$-phosphorene and arsenene, the stability of antimonene and bismuthene in the washboard phase occurred only in the asymmetric form. In Fig. 2, we present the optimized, stable geometries of these four monolayer structures. In Table I, we tabulate their structural parameters and elastic constants which were reported in previous studies. The cohesive energy and formation energy values at $\mathrm{T}=0 \mathrm{~K}$ reported in various papers are critical for the stability of pnictogen monolayers, which are given in the first column of Table II.

Apart from these four types of monolayer structures, recent studies indicated different allotropes. ${ }^{70-74}$ Several possible 2D phosphorene allotropes have been proposed over the recent years; most of them are 3-fold coordinated. ${ }^{75-82}$ Selected $\mathrm{sp}^{3}$ bonded phosphorene allotropes investigated by Guan et al., and they categorized the allotropes with structural index $N$ (tilling parameters). ${ }^{75}$ For instance, in the $h b-\mathrm{P}$ structure, the top phosphorus atom bonds with three bottom positioned phosphorus atoms and its tilling parameter is $N=0$. However, in the $s w$-P structure, the top positioned phosphorus atom bonds with two neighboring, top positioned phosphorus atoms, and hence, its tilling parameter is $N=2$. Their study showed that phosphorus atoms prefer to form zigzag chains at the same height, and so, energetically most stable structures have $N=2$ or $N=0 .{ }^{75}$ Yu et al ${ }^{82}$ theoretically showed that phosphorus atoms can be stable with the Kagome-like lattice (Kagome-P). Phonon dispersion of Kagome-P has a positive frequency in the whole Brillouin-Zone; however, it is less stable than $h b$ $\mathrm{P}$ and $s w$-P by 0.11 and $0.12 \mathrm{eV} /$ atom, respectively. Most recently, Liu et al $^{83}$ predicted new allotropes of phosphorene, which have 2-, 3and 4-fold coordination. ${ }^{83}$ Theoretical studies have also demonstrated that stable phosphorene phases may exist with chirality. ${ }^{83-86}$ Stability investigation of four allotropes $\left(h b_{-}, s w_{-}, \gamma-\right.$, and $\delta$-As) of $2 \mathrm{D}$ arsenic structures indicated that $\delta$-As has large negative frequencies near the $\Gamma$ point, and energetically $s w$-As is the most stable structure among the others. ${ }^{87}$ However, for the antimony allotropes both of $\gamma$ - and $\delta$-Sb have large negative frequencies in the phonon dispersion. ${ }^{88}$

Structure optimizations by themselves cannot assure the stability of free standing group-VA monolayers, even if the final structures have positive cohesive energies. The (dynamical) stability of the structures at $\mathrm{T}=0 \mathrm{~K}$ will be assured if vibrational frequencies calculated for all $\boldsymbol{k}$-points are positive. Otherwise, imaginary frequencies would imply structural instability for the corresponding modes. Calculated 

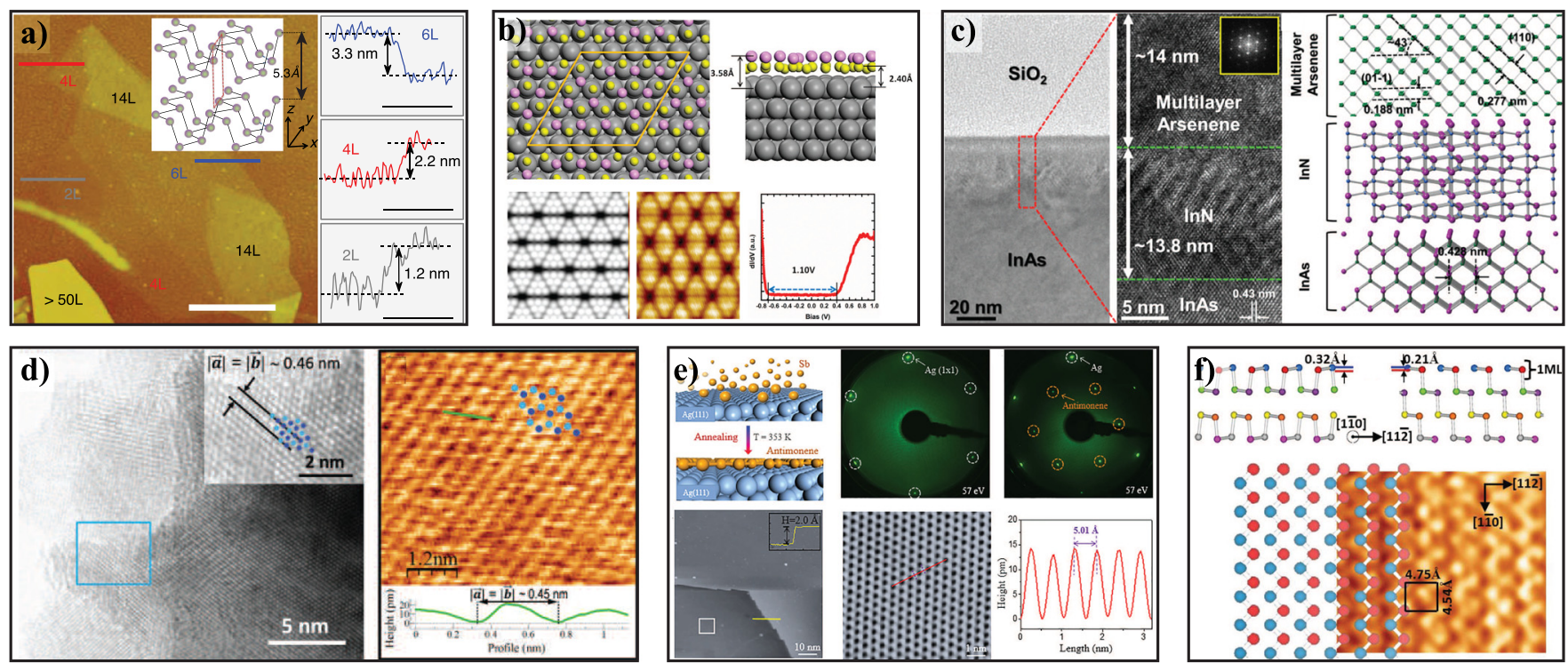

FIG. 1. (a) Left: an AFM image of black phosphorus flakes with estimated layer numbers. Scale bar (thick white) is $2 \mu \mathrm{m}$. Right: line scans performed along the colored lines shown in the left, where layer numbers from 2 to $>50$ are identified. The scale bar (gray) is $1 \mu \mathrm{m}$. Reproduced with permission from Xia et al. Nat. Commun. 5, 5458 (2014). Copyright 2014 Springer Nature. (b) Upper panel: DFT results for single layer blue phosphorene on Au(111). Bottom panel: Scanning Tunneling Microscope (STM) image simulated according to the model displayed in the panel is given at the left-hand side, experimentally observed STM image and dI/dV curve taken at the single layer blue phosphorus, revealing a bandgap around 1.10 V is given in the right-hand side. Reproduced with permission from Zhang et al. Nano Lett. 16(8), 4903-4908 (2016). Copyright 2016 American Chemical Society. (c) Left: TEM image of multilayer arsenene/InN/InAs (inset: diffraction pattern of multilayer arsenene) Right: theoretical atomic model of the multilayer arsenene/lnN/InAs layer structure. Reproduced with permission from Tsai et al. Chem. Mater. 28(2), 425-429 (2016). Copyright 2016 American Chemical Society. (d) High-resolution TEM image of a few-layer antimonene flake. The inset shows a digital magnification of the area inside the blue rectangle. Reproduced with permission from Ares et al. Adv. Mater. 28, 6332-6336 (2016). Copyright 2016 John Wiley and Sons. (e) Upper panel: from left to right; schematic of the fabrication process, LEED pattern of a clean $\mathrm{Ag}(111)$ substrate, presenting sharp $(1 \times 1)$ diffraction spots, respectively. Bottom panel: from left to right; LEED pattern of antimonene on $\mathrm{Ag}(111)$, presenting a $\mathrm{Ag}(111)-(\sqrt{3} \times \sqrt{3})$ superstructure, large scale STM image of monolayer antimonene on the $\mathrm{Ag}(111)$. Inset: a height profile along the yellow line at the terrace edge. The height corresponds to the intrinsic height of the $\mathrm{Ag}(111)$ terrace, suggesting that $\mathrm{Ag}(111)$ terraces are covered fully by a monolayer antimonene, high-resolution STM image of antimonene depicted by white square in left panel, demonstrating a well-ordered buckled honeycomb like lattice; the line profile corresponding to the red line in the left panel, revealing the periodicity of the antimonene lattice $(5.01 \AA)$. Reproduced with permission from Shao et al. Nano Lett. 18, 2133-2139 (2018). Copyright 2018 American Chemical Society. (f) Atom resolved STM image $\left(V_{T}=0.2 \mathrm{~V}\right)$ of the $\mathrm{Bi}(100)$ nanoribbon terrace superimposed by the single-layer Bi(100) atomic model. Reproduced with permission from Sun et al. Phys. Rev. Lett. 109, 246804 (2012). Copyright 2012 American Physical Society.
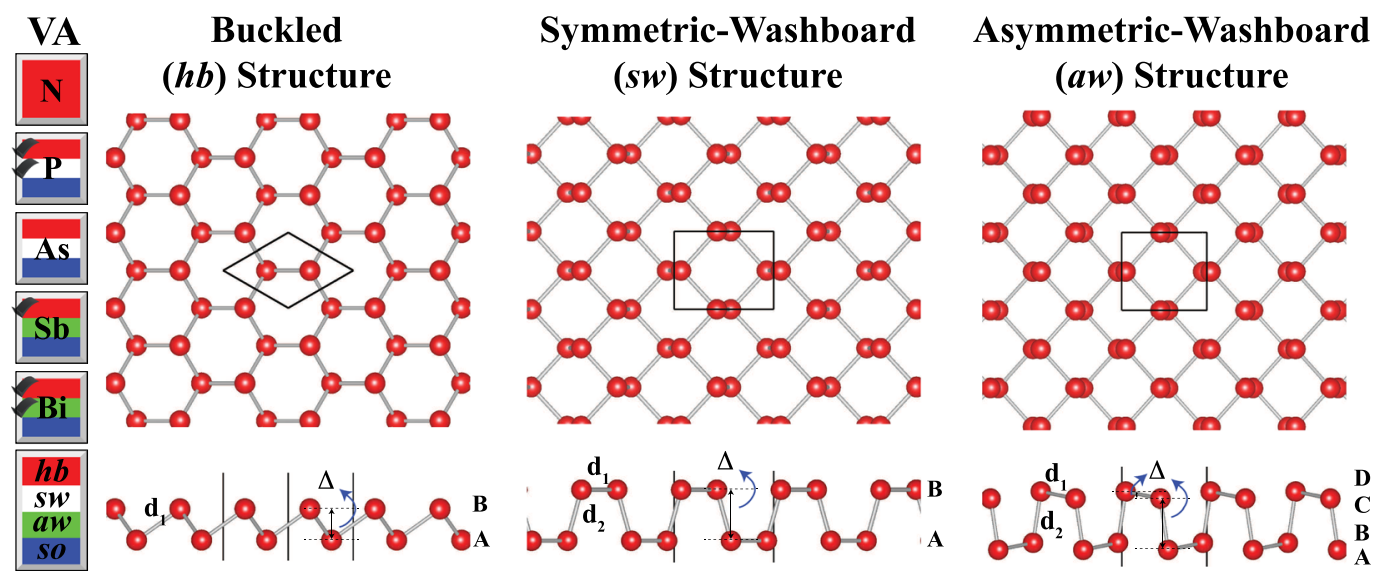

\section{Square-Octagon \\ (so) Structure}
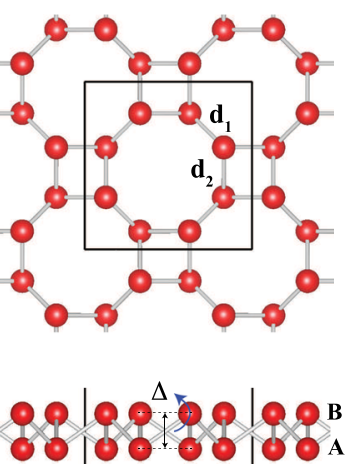

FIG. 2. Left panels summarize the results of stability analyses of group-VA elements that can form stable, $2 \mathrm{D}$ monolayer of $h b$-, sw- or aw-, and so-structures. Monolayers which are already synthesized are marked. The right panels display the top and side views of the optimized atomic structures. 2D hexagonal and rectangular unit cells are delineated. 
TABLE I. Suspended 2D monolayer structures of group-VA elements (N, P, As, Sb, and Bi): 2D structure phase (hb; buckled honeycomb, sw; symmetric washboard, aw; asymmetric washboard, and so; square-octagon); optimized lattice constants alb; bond lengths $d_{1} / d_{2}$; buckling height $\Delta$; in-plane stiffness $C_{x} / C_{y}$; and Poisson's ratio $\nu_{x y} / \nu_{y x}$.

\begin{tabular}{|c|c|c|c|c|c|c|}
\hline & Structure & $\begin{array}{l}\text { Lattice } \\
(\AA)\end{array}$ & $\begin{array}{c}\mathrm{d}_{i} \\
(\AA)\end{array}$ & $\begin{array}{c}\Delta \\
(\AA)\end{array}$ & $\begin{array}{c}\mathrm{C}_{x}=\mathrm{C}_{y} \\
\left(\mathrm{~J} / \mathrm{m}^{2}\right)\end{array}$ & $\nu_{x y}=\nu_{y x}$ \\
\hline $\mathrm{N}$ & $\begin{array}{l}h b \\
\text { so }\end{array}$ & $\begin{array}{c}a=2.27-2.30^{44,90} \\
a=4.42^{72}\end{array}$ & $\begin{array}{c}d=1.49-1.50^{44,90} \\
d_{1}=1.40^{72} \\
d_{2}=1.52^{72}\end{array}$ & $\begin{array}{c}0.70^{44,90} \\
0.70^{72}\end{array}$ & $270^{44}$ & $\ldots$ \\
\hline $\mathrm{P}$ & $\begin{array}{l}h b \\
s w\end{array}$ & $\begin{array}{c}a=3.28-3 \cdot 33^{49,91,92} \\
a=3.30-3 \cdot 36^{49,76,94,95} \\
b=4.53-4 \cdot 62^{49,76,94,95} \\
a=6.46-6.54^{49,72}\end{array}$ & $\begin{array}{c}2.26-2.27^{49,91,93} \\
\mathrm{~d}_{1}=2.22^{94,95} \\
\mathrm{~d}_{2}=2.25-2.26^{94,95} \\
d_{1}=2.26^{49,72} \\
d_{2}=2.28^{49,72}\end{array}$ & $\begin{array}{c}1.23,1.24^{49,91,93} \\
2.12-2.51^{49,95} \\
1.25^{49,72}\end{array}$ & $\begin{array}{c}\mathrm{C}_{x, y}=75.45^{49} \\
\mathrm{C}_{x}=41-44^{95,96} \\
\mathrm{C}_{y}=106-166^{95,96} \\
\mathrm{C}_{x, y}=32.06^{49}\end{array}$ & $\begin{array}{l}\nu_{x y}=0.11^{49} \\
\nu_{x y}=0.17^{95} \\
\nu_{y x}=0.62^{95} \\
\nu_{x y}=0.53^{49}\end{array}$ \\
\hline As & $\begin{array}{l}h b \\
s w\end{array}$ & $\begin{array}{c}a=3.60-3 \cdot 61^{45,49,97-99} \\
a=3 \cdot 67-3 \cdot 69^{45,49,99} \\
b=4.72-4.77^{45,49,99} \\
a=7.06-7.13^{49,72,99}\end{array}$ & $\begin{array}{c}2.45-2.51^{45,49,87,97-100} \\
d_{1}=2.49-2.50^{45,49,99} \\
d_{2}=2.47-2.51^{45,49,99} \\
d_{1}=2.48-2.50^{49,72,99} \\
d_{2}=2.52-2.53^{49,72,99}\end{array}$ & $\begin{array}{c}1.35-1.40^{49,87,97,100} \\
2.93-3.13^{45,49,87,99}-0.17^{49} \\
1.42^{49,72}\end{array}$ & $\begin{array}{c}\mathrm{C}_{x, y}=51.41^{49} \\
\mathrm{C}_{x}=29^{101} \\
\mathrm{C}_{y}=74.7^{101} \\
\mathrm{C}_{x, y}=20.87^{49}\end{array}$ & $\nu_{x y}=0.599^{49}$ \\
\hline $\mathrm{Sb}$ & $\begin{array}{l}h b \\
a w\end{array}$ & $\begin{array}{c}a=4.01-4.12^{46,102,103} \\
a=4.27-4.36^{46,103} \\
b=4.48-4.78^{46,103} \\
a=8.01-8.13^{49,72}\end{array}$ & $\begin{array}{c}2.87-2.89^{46,49,102} \\
d_{1}=2.85-2.87^{46,103} \\
d_{2}=2.91-2.94^{46,103} \\
d_{1}=2.85-2.89^{49,72} \\
d_{2}=2.91^{49,72}\end{array}$ & $\begin{array}{c}1.65-1.67^{46,49,102} \\
0.38,2.82^{49} \\
1.66-1.67^{49,72}\end{array}$ & $\begin{array}{c}\mathrm{C}_{x, y}=34.56^{49} \\
\mathrm{C}_{x}=12^{46} \\
\mathrm{C}_{y}=29^{46} \\
\mathrm{C}_{x, y}=11.55^{49}\end{array}$ & $\begin{array}{c}\nu_{x y}=0.192^{49} \\
\nu_{x y}=0.36^{46} \\
\nu_{y x}=1.20^{46} \\
\nu_{x y}=0.663^{49}\end{array}$ \\
\hline $\mathrm{Bi}$ & $\begin{array}{l}h b \\
a w\end{array}$ & $\begin{array}{c}a=4.33-4.45^{48,102,104} \\
a=4.87-4.94^{48,49} \\
b=4.44-4.55^{48,49} \\
a=8.40-8.74^{49,72,105}\end{array}$ & $\begin{array}{c}3.07-3.09^{48,102} \\
d_{1}=3.08-3.11^{48,49} \\
d_{2}=3.03-3.10^{48,49} \\
d_{1}=2.99-3.05^{49,72,105} \\
d_{2}=3.06-3.08^{49,72,105}\end{array}$ & $\begin{array}{c}1.71-1.74^{48,102} \\
0.5-0.57,2.72^{48,49} \\
1.74-1.78^{49,72,105}\end{array}$ & $\begin{array}{c}\mathrm{C}_{x, y}=23.9-29.6^{48,49} \\
\mathrm{C}_{x}=10.03^{48} \\
\mathrm{C}_{y}=25.50^{48} \\
\mathrm{C}_{x, y}=7.6149\end{array}$ & $\begin{array}{c}\nu_{x y}=0.23-0.33^{48,49} \\
\nu_{x y}=0.261^{48} \\
\nu_{y x}=0.65^{48} \\
\nu_{x y}=0.73^{49}\end{array}$ \\
\hline
\end{tabular}

dispersions of vibration frequencies of the free standing $h b$-, $s w$-, $a w$-, and so-monolayer structures of group-VA elements are presented along the major symmetry axis in Fig. 3. Overall features are similar in the same types of structures. Generally, as the row number of an element decreases, the corresponding bond length becomes shorter, and hence, the force constant becomes stronger, leading to relatively high vibrational frequencies. In Fig. 3, the vibrational frequencies of stable structures are all positive. In particular, the frequencies of acoustical and flexural out-of plane (ZA) modes are positive as $\mathbf{k} \rightarrow 0$. This ensures stability even for long wavelength lattice vibrations. Notably, structure optimization calculations ${ }^{49,50}$ predict that initially created $a w$-phases of $\mathrm{P}$ and As spontaneously transform to the $s w$-phases upon the relaxation as depicted in Fig. 3.

The gaps between the acoustical and optical modes have a close bearing on the thermal resistivity of the structures. The character of this gap results in different relaxation times in phonon scattering processes between the two structures of the same element, leading to different thermal conductivities and thermoelectric efficiencies. ${ }^{89}$ Except nitrogene, the optical branches are well-separated from the acoustical ones.

\section{A. Nitrogene}

Nitrogene ${ }^{44}$ with a $h b$-structure (similar to silicene or germanene ${ }^{8,9}$ ) has a lattice constant of $2.27 \AA$, which is smaller than the lattice constant of graphene. In nitrogene, alternating $\mathrm{N}$ atoms at the corners of the hexagon are placed in different planes. The height of buckling is $0.70 \AA$, which is larger than the buckling in silicene and germanene. ${ }^{9}$ Each $\mathrm{N}$ atom has three folded coordination and is bound to its three nearest neighbors with $s p^{3}$-like hybrid orbitals. ${ }^{44,90}$ The cohesive energy of nitrogene is $3.67 \mathrm{eV} /$ atom. ${ }^{44}$ Its stability is ensured through dynamical and thermal stability analyses. The calculated frequencies of vibration modes in the Brillouin zone and phonon dispersion curves along the symmetry directions presented in Fig. 3 are all positive. This indicates the stability at $\mathrm{T}=0 \mathrm{~K}^{44,106}$ Peng et al. ${ }^{114}$ pointed out that the group velocity of graphene is much higher than that of nitrogene. Accordingly, the lattice thermal conductivity of nitrogene $(763.4 \mathrm{Wm} / \mathrm{K})$ is nearly one fifth of graphene $(3716.6 \mathrm{Wm} / \mathrm{K}){ }^{114}$ Whether the $h b$-structure of nitrogene corresponds to a local minimum in the Born-Oppenheimer surface to sustain the thermal excitations can be revealed by finite-temperature ab-initio molecular dynamics (MD) simulations. These simulations showed that the $h b$-structure remains stable at $850 \mathrm{~K}$, yet it starts to disassemble into $\mathrm{N}_{2}$ molecules at $\mathrm{T}=1000 \mathrm{~K} \cdot{ }^{44}$ As for the mechanical properties, the in-plane stiffness of $h b$-nitrogene was calculated to be $\sim 270 \mathrm{~J} / \mathrm{m}^{2},{ }^{44,114}$ which is higher than the in-plane-stiffness of $2 \mathrm{D} h-\mathrm{MoS}_{2}\left(138 \mathrm{~J} / \mathrm{m}^{2}\right)$, even higher than that of $2 \mathrm{D} h$-BN $\left(240 \mathrm{~J} / \mathrm{m}^{2}\right)$. The washboard and square-octagon structures of nitrogene underwent an instability by dissociating into $\mathrm{N}_{2}$ molecules 
TABLE II. Energetics and bandgaps of free-standing monolayers of group-VA elements (N, P, As, Sb, and Bi): the type of structure (hb; buckled honeycomb, sw; symmetric washboard, aw; asymmetric washboard, and so; square-octagon); cohesive energy/formation energy per atom $E_{d} / E_{f}$, bandgap values obtained by PBE, ${ }^{134}$ PBE $+S O C$, $\mathrm{HSE},{ }^{135} \mathrm{HSE}+\mathrm{SOC}$, and QPGW with indirect/direct bandgap denoted by $(i) /(d)$. Exciton binding energies are denoted by $E_{b}$.

\begin{tabular}{|c|c|c|c|c|c|c|c|}
\hline \multicolumn{2}{|c|}{ Structure } & \multirow{3}{*}{$\begin{array}{c}\begin{array}{c}E_{c} / E_{f} \\
(\mathrm{eV})\end{array} \\
3.67 /-1.33^{44} \\
6.45^{72} / \ldots\end{array}$} & \multirow{3}{*}{$\begin{array}{c}\begin{array}{c}\mathrm{PBE} \\
(\mathrm{eV})\end{array} \\
(i) 3.70-3.96^{44,90,106} \\
(i) 2.61^{72}\end{array}$} & \multirow{3}{*}{$\begin{array}{c}\mathrm{PBE}+\mathrm{SOC} \\
(\mathrm{eV}) \\
(i) 3.70^{90} \\
(i) 2.61^{72}\end{array}$} & \multirow{3}{*}{$\begin{array}{c}\begin{array}{c}\text { HSE } \\
(\mathrm{eV})\end{array} \\
(i) 5.90^{44}\end{array}$} & \multirow{2}{*}{$\begin{array}{c}\mathrm{HSE}+\mathrm{SOC} \\
(\mathrm{eV})\end{array}$} & \multirow{2}{*}{$\begin{array}{c}\begin{array}{c}\mathrm{QP} G W / E_{b} \\
(\mathrm{eV})\end{array} \\
7.26^{44}\end{array}$} \\
\hline $\mathrm{N}$ & $h b$ & & & & & & \\
\hline & so & & & & & $\ldots$ & $\ldots$ \\
\hline \multirow[t]{3}{*}{$\mathrm{P}$} & $h b$ & $3.29-3.55^{49,76,107,108} /-0.12^{49}$ & (i) $1.91-1.98^{49,76,93}$ & (i) $1.97^{90}$ & $(d) 2.62^{70}$ & $\ldots$ & $\ldots$ \\
\hline & $s w$ & $3.30-3.59^{49,76,94} /-0.08^{49}$ & $(d) 0.82-1.00^{35,49,76}$ & $\ldots$ & (d) $1-1.83^{35,70,109}$ & $\ldots$ & $2.2 \pm 0.1^{142} / 0.7-0.9^{43,141,142}$ \\
\hline & so & $3.43-5.20^{49,72}$ & (i) $1.95-2.16^{49,72}$ & (i) $1.95-2.16^{49,72}$ & (i) $2.92^{49}$ & (i) $2.92^{49}$ & $\ldots$ \\
\hline \multirow[t]{3}{*}{ As } & $h b$ & $2.95-3.15^{45,49,87,97-99} /-0.16^{49}$ & (i) $1.57-1.64^{45,49,97}$ & (i) $1.81^{90}$ & (i) $2 \cdot 23-2 \cdot 51^{70,100,110,111}$ & $\ldots$ & $2.64 / 0.7^{159}$ \\
\hline & $s w$ & $2.93-3.13^{45,49,87,99} /-0.17^{49}$ & (i) $0.77-0.83^{45,49,87}$ & $\ldots$ & (i) $1.36-1.66^{70,110}$ & $\ldots$ & $1.58^{50} / \ldots$ \\
\hline & so & $2.86-4.50^{49,72,99}$ & (d) $1.70-1.79^{49,72}$ & (i) $1.59-1.68^{49,72}$ & $(d) 2.47^{49}$ & $(d) 2.32^{49}$ & $\ldots$ \\
\hline \multirow[t]{3}{*}{$\mathrm{Sb}$} & $h b$ & $2.86-4.57^{46,49,103}$ & (i) $0.76-1.18^{49,103,112}$ & ${ }^{2}$ (i) $1-1.04^{46,90}$ & (i) $1.55-2.28^{46,100}$ & $\ldots$ & $2.25^{174}$ \\
\hline & aw & $2.88-4.63^{46,49,103} /-0.24^{46,49}$ & (i) $0.15-0.28^{46,49,103}$ & $(d) 0.19^{46}$ & (d) $0.34^{46}$ & $\ldots$ & $0.37^{178} / \ldots$ \\
\hline & so & $2.69-3.91^{49,72} /-0.44^{49}$ & (d) $1.33-1.43^{49,72}$ & (d) $1.05-1.14^{49,72}$ & (d) $1.96^{49}$ & $(d) 1.63^{49}$ & $\ldots$ \\
\hline \multirow[t]{3}{*}{$\mathrm{Bi}$} & $h b$ & $1.95-2.77^{48,49,113,128} /-0.13^{48}$ & (d) $0.16-0.55^{48,49,102}$ & (i) $0.43-0.51^{48,90}$ & (d) $0.80-0.99^{48,100}$ & (i) $0.32^{48}$ & $0.92 / 0.18^{178} / \ldots$ \\
\hline & $a w$ & $1.97 /-0.12^{48}$ & (d) $0.29-0.31^{48,49}$ & (i) $0.28^{48}$ & (d) $0.23-0.36^{48,100}$ & (i) $0.39^{48}$ & $0.35^{178} / \ldots$ \\
\hline & so & $2.57-4.25^{49,72,105}$ & (d) $0.15-0.63^{49,72,105}$ & (i) $0.26-0.34^{49,72}$ & (d) $1.01^{49}$ & (d) $0.15^{49}$ & $\ldots$ \\
\hline
\end{tabular}

in the course of structure optimization. ${ }^{44,49}$ Nevertheless, Zhang et al. ${ }^{72}$ predicted that so-nitrogene can exist with a lattice constant of $4.42 \AA$, even if this is not confirmed by further stability analysis.

\section{B. Phosphorene}

Liu et al. ${ }^{35}$ and Lu et al. ${ }^{115}$ reported simultaneously that phosphorene can be obtained by the mechanical exfoliation method from its orthorhombic bulk phase. The lattice constants of black phosphorene, $s w$-P, shown in Table I are very close to those of the lattice constants of the bulk phase, $a=3.36 \AA$ and $b=4.53 \AA$, since the interaction energy between phosphorene layers are very weak $(20 \mathrm{meV} /$ atom $) .{ }^{35}$ Blue phosphorene, $h b$-P, which was predicted earlier, ${ }^{34}$ was epitaxially grown on $\mathrm{Au}(111) .{ }^{65}$ Inequivalent lattice constants $(\mathrm{a} \neq \mathrm{b})$ of $s w$-P lead to anisotropic thermal properties in contrast to isotropic $h b-\mathrm{P} .^{107,116}$ Another stable but not synthesized structure for phosphorus atoms is the sophase with square lattice constant and the buckling height, $\Delta=1.25$ $\AA^{49,72,75,79}$ Further details of P-phases and related properties can be acquired from several review papers specialized in phosphorene. ${ }^{52-60}$

\section{Arsenene}

It was theoretically predicted that arsenic can also be stable in its 2D form similar to phosphorene. Based on first-principles Density functional theory (DFT) calculations, Kamal and Ezawa ${ }^{45}$ showed that monolayer $h b$-As and $s w$-As are dynamically stable, which was confirmed later by Zhang et al. ${ }^{100}$ Also, as mentioned above, in the course of structure optimization at $\mathrm{T}=0 \mathrm{~K}$ with, the asymmetric washboard structure $a w$-As underwent a structural transition to the symmetric one, indicating the fact that $a w$-As is unstable. ${ }^{50}$ Incidentally, the equilibrium structures occurring at $\mathrm{T}=0 \mathrm{~K}$, can be changed by thermal excitations or by other means. For example, finite temperature, molecular dynamics simulations by Kecik et al. ${ }^{50}$ demonstrate that both arsenene structures maintain their forms, except thermally induced deformation. No bond breaking or clustering in the structures were observed up to $1000 \mathrm{~K}$, except for $s w$-As transforming into the $a w$-As structure at $600 \mathrm{~K}$ as a result of thermal excitation with minute asymmetrization. ${ }^{50}$ This situation indicates that the energy of $a w$-As is slightly above that of $s w$-As at $\mathrm{T}=0 \mathrm{~K}$. Also, recently, the antisymmetric structure of monolayer Arsenene was obtained by applied distortion of some As atoms in the cell. ${ }^{117}$ The distortion gives rise to the breaking of the centrosymmetry and constructs a spontaneous electric polarization. The calculated Curie temperature for the phase transition between $s w$-As and $a w$-As is $478 \mathrm{~K}$. This result is in compliance with the thermally induced, $s w \rightarrow a w$ transition of arsenene at $600 \mathrm{~K}$, obtained by Kecik et al..$^{50}$ Similar to phosphorene, arsenene is also stable in the so-structure. ${ }^{49,72}$ For $\mathrm{T} \sim 0 \mathrm{~K}$, the calculated cohesive energies are ordered $h b$-As $>s w$-As $>$ so-As. Recently, Carrete et al. ${ }^{99}$ predicted that $s w$-As and so-As are thermodynamically more favorable than the $h b$-As phase at low, as well as at high temperatures, respectively. In addition, thermal conductivity of $s w$-As and so-As is higher than that of the $h b$-As structure, since formers have more complex structures than the $h b$-phase. ${ }^{99}$ However, when the energy is calculated by considering the full harmonic free energy including the temperature-dependent energy and entropy terms, the stability order of arsenene allotropes undergoes a change. ${ }^{99} h b$-As shows also an excellent thermoelectric response at $700 \mathrm{~K} .{ }^{118}$ On the other hand, the thermal conductivity of $s w$-As is one third of that of $s w$-P, owing to its relatively large atomic mass. $^{119}$

\section{Antimonene}

The rhombohedral phase of bulk antimony $(\beta-\mathrm{Sb})$ is a semimetal and undergoes a series of transitions as the thickness of the material is reduced. A 22-layer Sb film behaves as a topological insulator, and an 8-layer slab attains a quantum spin Hall (QSH) phase; nonetheless, it 
turns out to be a semiconductor under three layers. ${ }^{120}$ Monolayer $h b$ Sb is stable for the buckling height $\Delta=1.67 \AA \AA^{46,62}$ Its hexagonal lattice constant is $a=4.04 \AA$, and in-plane stiffness $C=41 \mathrm{~J} / \mathrm{m}^{2}$, comparable to that of silicene $\left(65 \mathrm{~J} / \mathrm{m}^{2}\right){ }^{46}$ The Young modulus and shear modulus of $h b$-Sb were calculated to be $31 \mathrm{~J} / \mathrm{m}^{2}$ and $13 \mathrm{~J} / \mathrm{m}^{2}$, respectively. ${ }^{114}$ The asymmetric reconstruction in the washboard structure of antimonene provides the stability to form $a w$-Sb. ${ }^{46,102,103}$ Furthermore, finite temperature ab-initio, molecular dynamics simulations reveal that both $h b$ $\mathrm{Sb}$ and $a w$-Sb remain stable at $1000 \mathrm{~K}$ for $2 \mathrm{ps}^{46}$ The $s o-\mathrm{Sb}$ is predicted to be a stable monolayer structure sustaining thermal excitations. ${ }^{49,72}$ The cohesive energies of these three phases are ordered as $a w-S b>h b$ $\mathrm{Sb}>s o$-Sb, with the $a w$-structure marking the highest cohesive energy.

The thermoelectric performance of antimonene phases is studied by Chen et al., ${ }^{121}$ who found that among all pnictogen monolayers, $h b$-Sb has the highest thermoelectric efficiency, which can be enhanced under tensile strain. The thermal conductivity of pristine $h b-\mathrm{Sb}$ can be decreased by full hydrogenation or halogenation. ${ }^{122}$ In addition to functionalization, size and edge roughness affect the thermal conductivity of $h b$-Sb and $a w-S b .{ }^{123}$

\section{E. Bismuthene}

Bulk bismuth has a rhombohedral structure containing two $\mathrm{Bi}$ atoms per primitive cell with an experimentally determined lattice constant of $4.72 \AA^{124-126}$ although its free standing monolayer has a lattice constant of $4.33 \AA$ with the vertical distance between the two atomic planes, $\Delta=1.74 \AA{ }^{127}$ While $h b$-Bi is energetically favorable, it can transform into the metastable planar honeycomb structure. ${ }^{127}$ Similar to antimonene, $a w$-Bi has $5 \mathrm{meV}$ /atom higher cohesive energy in comparison to $s w$ - $\mathrm{Bi}^{48}$ The effect of spin-orbit coupling (SOC) on phonon bands for $h b$-Bi appears as down shifts ${ }^{129}$ of the optical frequencies by $\Delta \Omega=0.42 \mathrm{THz}$. Moreover, acoustical modes are slightly softened upon the inclusion of SOC. In fact, when the atomic structure and lattice constants are optimized concomitantly, $s w$-Bi transforms into $a w$-Bi spontaneously. A similar situation occurred for $s w$-Sb. ${ }^{46}$ Nonetheless, $s w$-Bi can be stable when it is placed on a substrate. ${ }^{48}$ Molecular dynamics calculations suggest that both $h b$-Bi and $a w$-Bi structures sustain to thermal excitations; however, irreversible distortions start to occur above $700 \mathrm{~K}^{48} s o-\mathrm{Bi}$ is also a stable structure and has the cohesive energy, which is only $0.20 \mathrm{eV} /$ atom lower than that of the $h b$-Bi phase. ${ }^{49}$ so-Bi has the largest lattice constant and the lowest Young modulus as compared to other so-structures of group-VA elements. ${ }^{49,72,105}$ Cheng et al. predicted that monolayer $h b$-Bi has larger power factors and lower thermal conductivity in comparison to its bulk phases, and hence, its thermoelectric performance would be superior to bulk $\mathrm{Bi}^{128}$ Similar to $h b$-Sb, the $h b$-Bi structure also has a high thermoelectric efficiency. ${ }^{128}$

\section{ELECTRONIC AND OPTICAL PROPERTIES}

Nitrogene, ${ }^{44}$ phosphorene, ${ }^{34}$ arsenene, ${ }^{45}$ antimonene, ${ }^{46}$ and bismuthene ${ }^{48}$ have recently gained importance due to their semiconducting properties. Furthermore, due to the large spin-orbit coupling effects shown by antimonene, bismuthene, and their compounds, ${ }^{48,131-133}$ these layered structures have attracted special interest owing to their possible topologically non-trivial states in specific conditions. Apparently, as the row number increases, the topological insulator properties stand out gradually. Applying transverse electric fields to arsenic allotropes induces band inversion at the $\Gamma$ point and leads to a topologically nontrivial phase in the band structures. ${ }^{87}$ A similar case can take place for antimonene by applying tensile $\operatorname{strain}^{88}$ or occurs by itself as for the bismuth allotropes. ${ }^{130}$ A comprehensive collection of the calculated energy bandgaps are presented in Table II in order to provide a comparison among the different approaches. In Fig. 4 , we present the calculated band structures of $h b-, s w^{-}, a w^{-}$, and $s o-$ phases of monolayer of $\mathrm{N}, \mathrm{P}, \mathrm{As}, \mathrm{Sb}$, and Bi along major high symmetry directions. In particular, washboard phosphorene and arsenene retain their $\Gamma$-valley band edges even when their layer numbers are increased. Therefore, one can envision various type-I or type-II $\Gamma$ valley lateral heterostructures by controlling the layer number. In addition, the puckered monolayers tend to have relatively low CBMs, $\sim$ $-4 \mathrm{eV}$ which allows them to form type I or II heterostructures with group-IV and III-V compounds. In a recent study, ${ }^{136}$ the band alignment properties of group-VA monolayers were investigated using HSE-SOC.

The linear optical response of solids can be calculated from the frequency-dependent complex dielectric function $\varepsilon_{i}(\omega)$, which is constituted of the real and imaginary parts: $\varepsilon(\omega)=\varepsilon_{1}(\omega)+\mathrm{i} \varepsilon_{2}(\omega)$. The imaginary part $\varepsilon_{2}(\omega)$ is determined by a summation of transition matrix elements over empty states wherefrom the real part of the dielectric tensor $\varepsilon_{1}$ can be derived using the Kramers-Kronig relationship. ${ }^{137}$

Efficient light absorber materials are required for various photovoltaic and optoelectronic applications to operate over a wide energy window, which need appropriate optical gap of that material. 2D group-VA monolayers with fundamental bandgaps near the infrared and visible regions have promising optoelectronic properties for future broadband photonic and light modulator device applications. Since especially the monolayer phases of arsenene, phosphorene, and antimonene were found to be promising due to their indirect and direct bandgaps which are resonant in the vicinity of the solar regime, their optical properties have been vastly investigated in several recent studies. From the methodology perspective, the optical spectra computed from random phase approximation (RPA) and the Bethe-Salpeter equation (BSE) on top of $\mathrm{PBE}, \mathrm{HSE}$, and quasiparticle $(\mathrm{QP}) G_{0} W_{0}$ methods are presented in the Fig. 5. The imaginary dielectric functions are presented for various structures of nitrogene, arsenene, antimonene, and bismuthene either with incident light polarized perpendicular to the c-axis (i.e., polarized along $x$ - or $y$-directions) or parallel to the c-axis (i.e., polarized along the $z$ direction). In Fig. 6, we present optical spectra of $a w$-P, $h b$-As, $s w$-As, $h b$-Sb, and $h b$-Bi computed by taking the many-body effects into account, obtained by employing the quasiparticle $G W$ approach, and also accounting for the electron-hole interactions using the BSE method. Upon excitation of the electrons and subsequent formation of holes, excitons are created via Coulomb interactions with certain binding energies. This situation constitutes the difference between the electronic and optical bandgaps. After these general remarks, we next review the electronic and optical properties of each monolayer structure.

\section{A. Nitrogene}

While pristine nitrogene is a nonmagnetic, a wide bandgap insulator with a DFT bandgap of $\mathrm{E}_{g}=3.70-3.96 \mathrm{eV}, 44,90,106$ its nanoribbons with zigzag and armchair edge geometries have bandgaps in the range of $0.6 \mathrm{eV}<\mathrm{E}_{g}<2.2 \mathrm{eV}$, which can be engineered by varying their widths. The variation of bandgap with different methods of corrections can be retrieved from Table II. A recently discovered allotrope of $2 \mathrm{D}$ nitrogene, the so-called octagon-nitrogene $(\mathrm{ON})$ was found to 
be a wide gap semiconductor with an indirect bandgap of $4.7 \mathrm{eV}$. Furthermore, the gap was decreased upon layer stacking, biaxial strain, and perpendicular electric field, where the latter two effects eventually lead to closing of the gap and insulator-to-metal transition. ${ }^{138}$

\section{B. Phosphorene}

Monolayer of black phosphorene, $s w$-P is renown with its direct bandgap around $0.9 \mathrm{eV}$ at the $\Gamma$ point, ${ }^{35,43,139,140}$ with anisotropic dispersion around this point for both valence and conduction bands (the dispersion is pronounced along the armchair direction, while the bands are rather flat along the zigzag direction). The effective mass was reported to be $m^{*}=0.15 m_{e}$ along the armchair direction, and $m^{*}=1.25 m_{e}$ along the zigzag direction. This anisotropy is further enhanced through the self-energy corrections and attributes a $1 \mathrm{D}$ effective dimensionality to carriers along the armchair direction. It was also found that by applying uniaxial or biaxial strain, the anisotropy in the electron effective mass and mobility can be rotated by $90^{\circ}$ in the reciprocal space, whereas the direction of anisotropy related to holes is not perturbed at all. ${ }^{140}$ First-principles simulations reveal that this is a result of a switch in the energy order of the lowest two conduction bands. This strain-engineered anisotropy in electrical properties makes $s w$-P even more promising for mechanical and electronic applications, such as stretchable electrical devices, mechanically controlled logical devices, and nanosized mechanical sensors. ${ }^{140}$

The photoluminescence peak of monolayer phosphorene, for light polarized along the $\Gamma$-X direction resides around $1.45 \mathrm{eV}$, according to the $G W$ calculation of Tran et al., ${ }^{43}$ with an exciton binding energy of $1.4 \mathrm{eV}$. Anisotropy of the optical response of $s w$-P is obvious from the difference in the spectra calculated for light polarized along the armchair and zigzag directions. They also found that for the zigzag direction, the absorption onset is observed near $2.8 \mathrm{eV}$, and hence, the spectral range of optical activity in $s w$ - $\mathrm{P}$ is tunable depending on the polarization direction in the lattice. Few-layer black phosphorus strongly absorbs light polarized along the armchair direction; however, it is transparent to light polarized along the zigzag direction. Moreover, $s w$-P is predominantly absorbent across the infrared and also partially in the visible light range, for incident light polarized along the armchair, $\Gamma$-X direction.

Tran et al. ${ }^{43}$ also revealed that the first absorption peak of $s w$-P is located at $1.2 \mathrm{eV}$, which is a strongly bound excitonic state with a $800 \mathrm{meV}$ e-h binding energy, similar to $700 \mathrm{meV}$ predicted by Cakir et al., ${ }^{141}$ as shown in Fig. 6(d). These exciton binding energies in phosphorene are comparable to those found in other monolayer semiconductors and 1D nanostructures. Wang et al. ${ }^{142}$ reported the experimental observation of highly anisotropic excitons with a large binding energy $(0.9 \pm 0.12 \mathrm{eV})$ in the monolayer of $s w$-P. The optical and quasiparticle bandgaps of monolayer black phosphorus were determined as $1.3 \pm 0.02$ and $2.2 \pm 0.1 \mathrm{eV}$, respectively. The exciton binding energy was measured as $0.3 \mathrm{eV}$ for the monolayer of $s w$-P on the $\mathrm{SiO}_{2} / \mathrm{Si}$ substrate. ${ }^{143}$

In summary, enhanced many-electron effects are essential in determining the bandgaps and optical response due to the anisotropic band dispersions and the effective quasi-1D nature. In particular, anisotropic optical response with e-h interactions included makes black phosphorene an ideal nanostructure for linear optical polarizers in the infrared and part of the visible light range.

\section{Arsenene}

Arsenene has high carrier mobility, superior mechanical properties, negative Poisson's ratio, and possible topological phase transition features. ${ }^{49,144-151}$ The range of its bandgap in Table II is interesting for flexible 2D nanoelectronics, optoelectronics, and photocatalytic applications. Progress towards the synthesis of arsenene phases has also been made. ${ }^{50,66,90}$

Both the electronic and optical properties of arsenene, which take into account the many-body, strain, electric field, and layer stacking effects, were studied to a significant extent much recently. ${ }^{152-158}$ For the $h b$-As monolayer, the optical absorption takes place within the spectral range of $2-8 \mathrm{eV}$ for the light polarized along $x$ - and $y$-directions and from $4 \mathrm{eV}$ to $9 \mathrm{eV}$ for the $z$-direction. While the HSE-RPA approach blueshifts the spectrum uniformly by nearly $0.5 \mathrm{eV}$ owing to its bandgap of $2.25 \mathrm{eV}, G_{0} W_{0}$-RPA spectrum onset is blueshifted to $\sim 2.6 \mathrm{eV}$. The $G_{0} W_{0}$-RPA imaginary dielectric function points to the main absorption peak focused in the range of $4 \mathrm{eV}-6 \mathrm{eV}$. Hence, with regard to $h b$-As, the optical absorption phenomenon is noteworthy within the visible to ultraviolet light range. Regarding out-of-plane absorption, the main peak is observed near $7.6 \mathrm{eV}$ for $G_{0} W_{0}$-RPA calculations. $^{159}$

The significant anisotropy along the directions of $\mathbf{a}$ and $\mathbf{b}$ lattice vectors within the washboard lattice is reflected to the optical properties, as shown in Fig. 5 Absorption onset appears earlier for $s w$-As than $h b$-As, for light polarized along the $x$-direction. Notably, while $s w$-P is well-known for its anisotropic properties, direct bandgap and pseudo-1D excitonic features, ${ }^{160,161}$ the anisotropic behaviors appear also in $s w$-As, $a w$-Sb, and $a w$-Bi. Specifically, the $\varepsilon_{x x}(\omega)$ components of the imaginary dielectric function of $s w$-As and $a w$-Sb point to a wide energy window of absorption, which can be a good basis for the design of optical modulator, photovoltaic, and optoelectronic materials/devices.

Prominent interband transitions are influential on the strong peaks in the $G_{0} W_{0}$-BSE spectrum. Although both buckled and washboard phases have indirect bandgaps, their corresponding direct gaps are shown by the red vertical lines in Fig. 6. An exciton binding energy, $E_{b}$ of the first peak in $h b$-As monolayer was estimated to be around $700 \mathrm{meV} .{ }^{159}$ Hence, it is comparable to those of few 2D semiconductors. Similar or slightly smaller exciton binding energies are given for $\mathrm{MoSe}_{2}, \mathrm{WS}_{2}$ and $\mathrm{WSe}_{2}$, which reveal $E_{b}$ as $450-500 \mathrm{meV}$ theoretically and $400-700 \mathrm{meV}$ experimentally, ${ }^{162-165}$ as well as phosphorene $(0.70-0.80 \mathrm{eV}) .{ }^{161,166}$ Yet, $E_{b}$ of arsenene monolayers are fairly smaller than that of $2 \mathrm{D} \mathrm{MoS}_{2}(1.1 \mathrm{eV}){ }^{167}$ The exciton binding energy of bilayer arsenene is reported as $\sim 500 \mathrm{meV}$ by Chaves et al. ${ }^{168} E_{b}$ is expected to decrease upon increasing the number of layers. For light polarized along the $y$ direction, $s w$-As is found more eligible for continuous absorption across the solar spectrum. The large modifications in the many-body optical properties of arsenene could allow for diverse photovoltaic and optoelectronic applications.

\section{Antimonene}

Antimonene gained particular significance due to the following reasons: (i) the range of fundamental bandgap of antimonene is promising for potential applications in 2D electronic and optical nanodevices. $^{46,169-174}$ (ii) Nanoribbons of $h b$-Sb are semiconductors with bandgaps varying upon chirality and width. ${ }^{175,176}$ Likewise, the 
a)

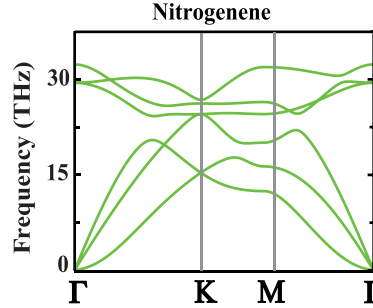

b)

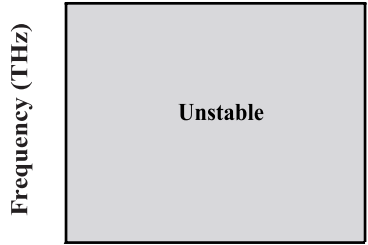

c)

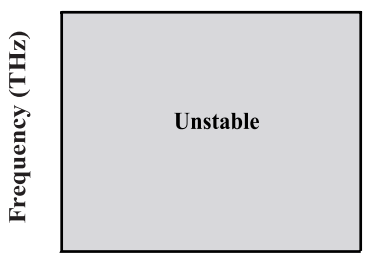

d)

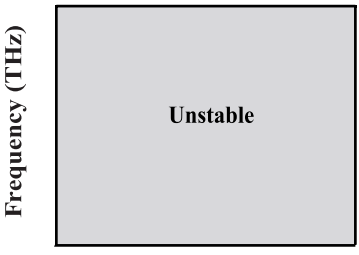

Phosphorene
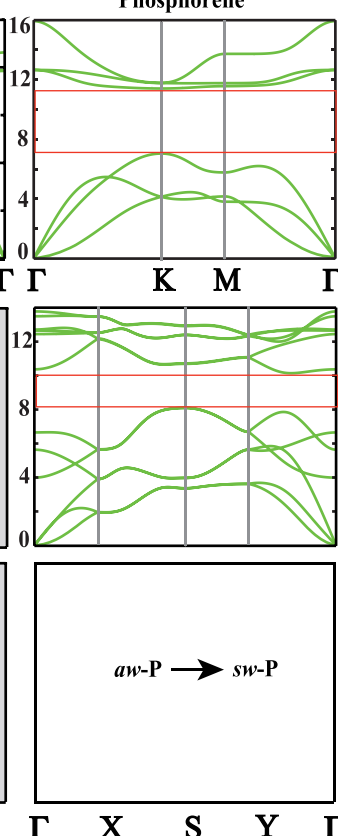
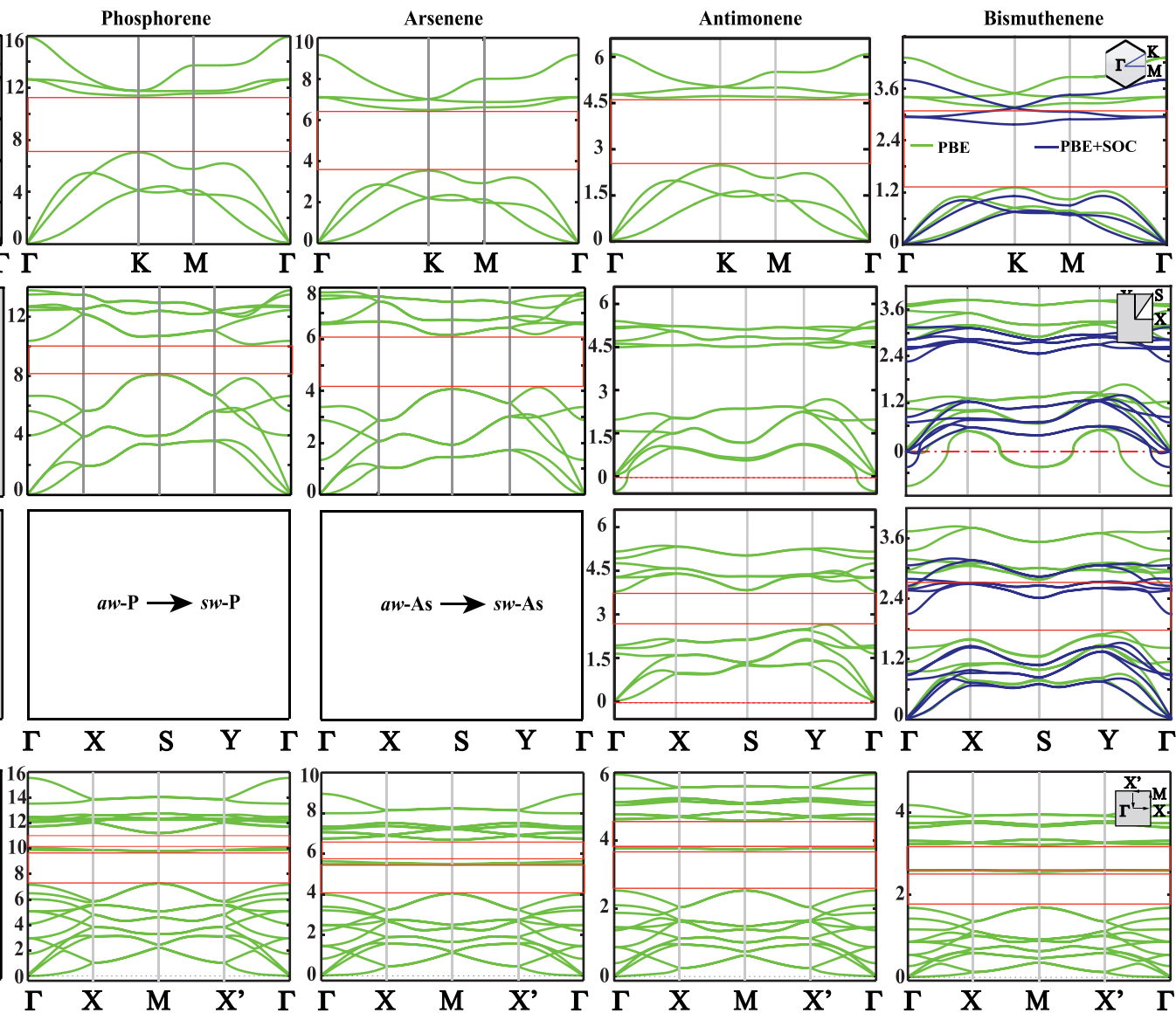

FIG. 3. Calculated phonon dispersion curves of $2 \mathrm{D}$ monolayers of group-VA elements. (a) $h b-;$ (b) $s w$-; (c) aw-; and (d) so-structures. The phononic bandgaps between the acoustical and optical branches are highlighted. Reproduced with permissions from Özçelik et al. Phys. Rev. B 92, 125420 (2015); Aktürk et al. Phys. Rev. B 91, 235446 (2015); Aktürk et al. Phys. Rev. B 94, 014115 (2016); Ersan et al. Phys. Rev. B 94, 245417 (2016); Kecik et al. Phys. Rev. B 94, 205409 (2016). Copyright 2015-2016 American Physical Society.

bandgap of $a w$-Sb nanoribbons vary also with their width, offering a tunability of the bandgap. (iii) The Sb element can form stable monolayers of binary compounds, which are also semiconductors with a fundamental bandgap different from monolayers of parent constituents. Some of these 2D compounds exhibit topologically non-trivial behaviors under specific conditions. ${ }^{133,177}$

The electronic and optical properties of antimonene monolayers have been studied intensely, focusing on more than one phases, using different theoretical approaches. ${ }^{46,90,155,178}$ Recently, Kripalani et al. ${ }^{179}$ found that the nature of the bandgap remains insensitive to strain in the zigzag direction, while strain in the armchair direction activates an indirect-direct bandgap transition at a critical strain of $4 \%$, owing to a band switching mechanism.

The optical properties of antimonene have been studied vastly. ${ }^{153,155,171,173,174,180}$ Alongside the DFT ${ }^{171}$ and HSE calculations (with random-phase approximation employed subsequently) of $h b$ and $a w$-Sb examined by Xu et al., ${ }^{173}$ Shu et al. ${ }^{174}$ studied the optical properties of $h b$-Sb using a many-body approach, under the effect of the electric field and strain. They pointed to a strong electric field closing the bandgap, as well as an increasing strain level leading to a redshift of the optical absorption spectra, with enhanced optical absorption within $1.2-2.2 \mathrm{eV}$ for $a w$-Sb. While they found the $G_{0} W_{0}$ bandgap of $a w$-Sb as indirect and $2.25 \mathrm{eV}$, an exciton binding energy of $0.73 \mathrm{eV}$ was calculated. The optical properties of monolayer $h b$-Sb can also be tuned via surface charge doping by $\mathrm{p} / \mathrm{n}$ type surface dopants, which revealed enhanced absorption of light especially in the early photon energy regime. ${ }^{153}$ The optical and luminescence bandgaps of the $h b$-Sb monolayer was found as $2.3 / 1.5 \mathrm{eV}$, leading to an exciton binding energy of $0.8 \mathrm{eV} .{ }^{155}$ Promising indications of broadband use of antimonene as a photodetector or optical modulator have been shown recently. ${ }^{181,182}$ It was commented earlier by Xu et al. ${ }^{183}$ that significant absorption from the visible region to the ultraviolet region can be observed in $a w$-Sb, while Ares et al. ${ }^{62}$ reviewed its properties and commented on $\mathrm{Sb}$ to have a suitable tunable bandgap for use in optoelectronics. In another study by Singh et al., ${ }^{171}$ it was found via the dielectric function and electron loss spectra that plasmon energies around $9 \mathrm{eV}$ and parts of the electromagnetic spectrum make antimonene behave like a metal in terms of reflectivity of light. 

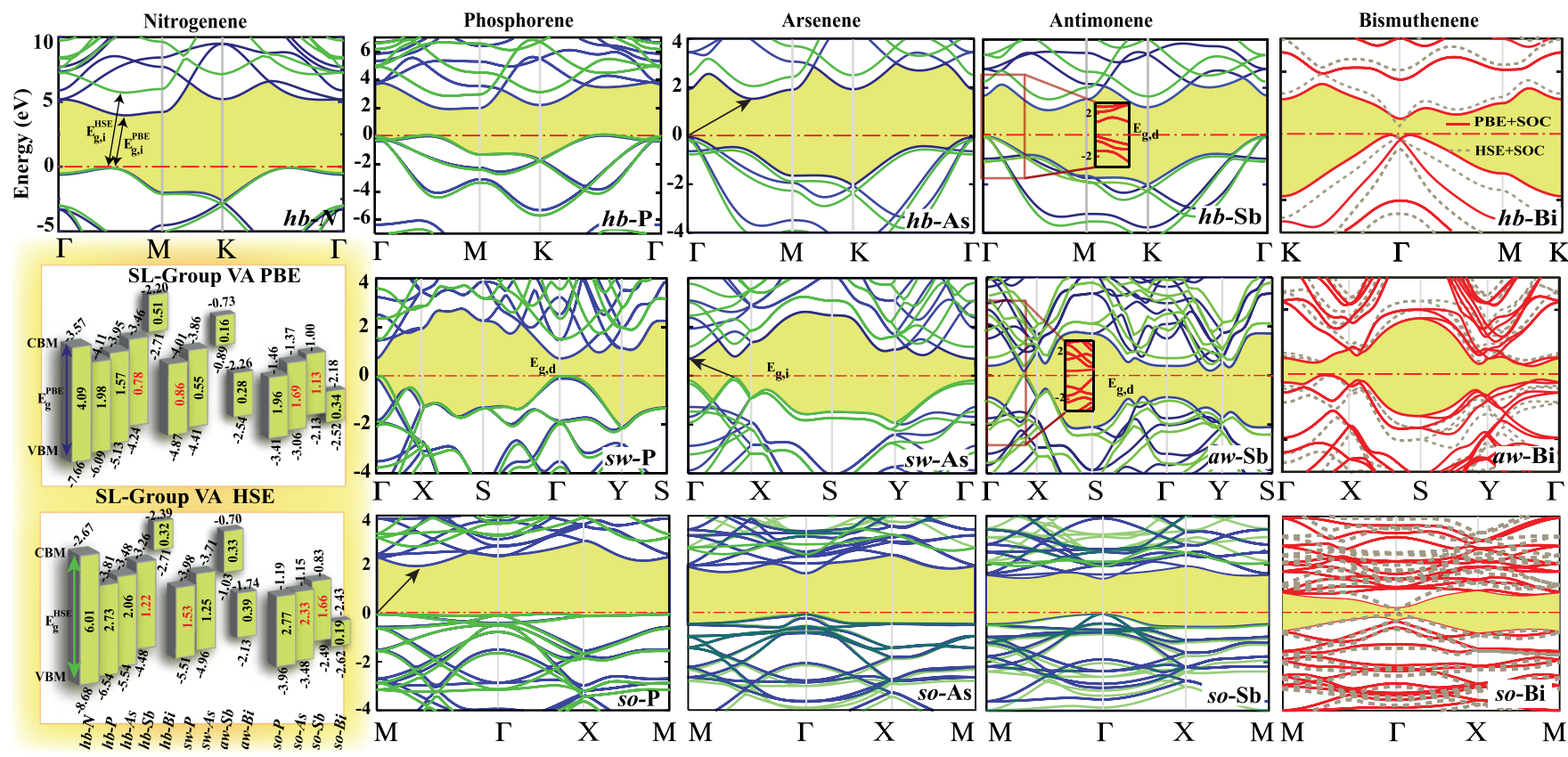

FIG. 4. Top row: electronic energy band structure of $2 \mathrm{D}$ group-VA monolayers (SL) calculated by PBE without SOC (blue-dark lines) and HSE without SOC (green-light lines) are presented along the major symmetry directions of the hexagonal Brillouin zone. Direct, $E_{g d}$ and inverted indirect $E_{g i}$ bandgaps are shown by the inset. Middle row: electronic energy bands of washboard structures calculated by PBE with SOC (red-dark lines) and HSE with SOC (dashed gray-light lines). Bottom row: energy bands of squareoctagon structures calculated by PBE with SOC (red-dark lines) and HSE with SOC (dashed gray-light lines). In the left-hand side, band alignments of monolayers (SL) with conduction band minimum and valence band maximum (CBM and VBM) and bandgaps values obtained from PBE and HSE calculations are shown. Reproduced with permissions from Özçelik et al. Phys. Rev. B 92, 125420 (2015); Aktürk et al. Phys. Rev. B 91, 235446 (2015); Aktürk et al. Phys. Rev. B 94, 014115 (2016); Ersan et al. Phys. Rev. B 94, 245417 (2016). Copyright 2015-2016 American Physical Society.

As presented in Fig. 5, $h b$-Sb with the onset of absorption residing at $\sim 2 \mathrm{eV}$, optical activity extending beyond the visible regime can be considered as a significant candidate $2 \mathrm{D}$ material for devices operating under both visible and ultraviolet light. ${ }^{178}$ Kecik et al. reported that the SOC effect on the optical properties of $h b$-Sb appears to be minor. ${ }^{178}$ Owing to the tiny bandgap of $a w$-Sb, one observes no clear onset of the absorption band edge. A main difference with the $h b$-Sb's optical response is the anisotropy along $x$ - and $y$-directions in the lattice. The effect of SOC on the in-plane optical responses generally refers to an extra early smaller peak with no major modification of the overall features. As shown in Fig. 6, a major excitonic peak for $h b$-Sb is observed slightly above $2.2 \mathrm{eV}$, where no apparent exciton binding is evident. $^{178}$

\section{E. Bismuthene}

The quest for $2 \mathrm{D}$ topological insulators has made the electronic structure of 2D ultrathin films, as well as single-layers (SLs) of Bi and BiSb compound a focus of interest. ${ }^{46,48}$ While 3D Bi crystal with a small direct bandgap and inverted indirect bandgap is a semimetal, it becomes a topological insulator under strain. ${ }^{131}$ Notably, thin films of Bi crystal grown on selected substrates revealed $2 \mathrm{D}$ topological insulator behavior together with surface and edge states. ${ }^{184-191}$ Also, $h b-\mathrm{Bi}$ placed on the $\mathrm{Si}(0001)$ substrate was shown to possess a topologically non-trivial phase. ${ }^{192}$ Kadioglu et al. ${ }^{132}$ found that while the pristine $h b$-Bi monolayer displays topologically non-trivial behavior, $a w-B i$ does not. Not only pristine and bare Bi ultrathin films and monolayers, but also the topological behaviors of defected Bi structures have been subject of studies. Freitas et al. ${ }^{193}$ studied the effect of $\mathrm{H}$ covered $\mathrm{Bi}$ surfaces on the topological character of the material. Earlier, calculations of the band structure of thin (one to six bilayers) of $h b$ - $\mathrm{Bi}$ in (111) and (110) orientations predicted that electronic properties range from small bandgap semiconductor to semimetal and metal. ${ }^{194}$

The optical properties of bismuthene have been investigated to a limited extent, with few recent examples on electric field applications and its potential as a photonic based device, ${ }^{153,195-197}$ hence the lightmatter interaction of $2 \mathrm{D} \mathrm{Bi}$ remains an open area of interest. Furthermore, although the dynamical stability, electronic structure, and topological features of BiSb binary compound monolayer have been investigated widely, ${ }^{133,177,198-202}$ little is known about their optical properties. Xiao et al. ${ }^{198}$ investigated systematically the stable binary compounds of the $2 \mathrm{D}$ BiSb monolayer, where they revealed few stable $a w$-BiSb alloy structures, one with a direct HSE bandgap of $0.43 \mathrm{eV}$. The effects of strain on the stability and electronic properties of BiSb compound monolayer were examined, and it turned out to be a direct gap semiconductor, reducing upon strain, with direct-indirectmetal transition under compressive strain. ${ }^{199}$ When SOC was included, a Rashba spin-splitting of $13 \mathrm{meV}$ in $h b$-BiSb near the Fermi level was observed, that can be tuned by in-plane biaxial strain, promising for efficient spin field-effect transistors, optoelectronics, and spintronic devices. ${ }^{177}$ The same material was predicted to become the quantum spin Hall insulator under biaxial strain. ${ }^{200}$ 

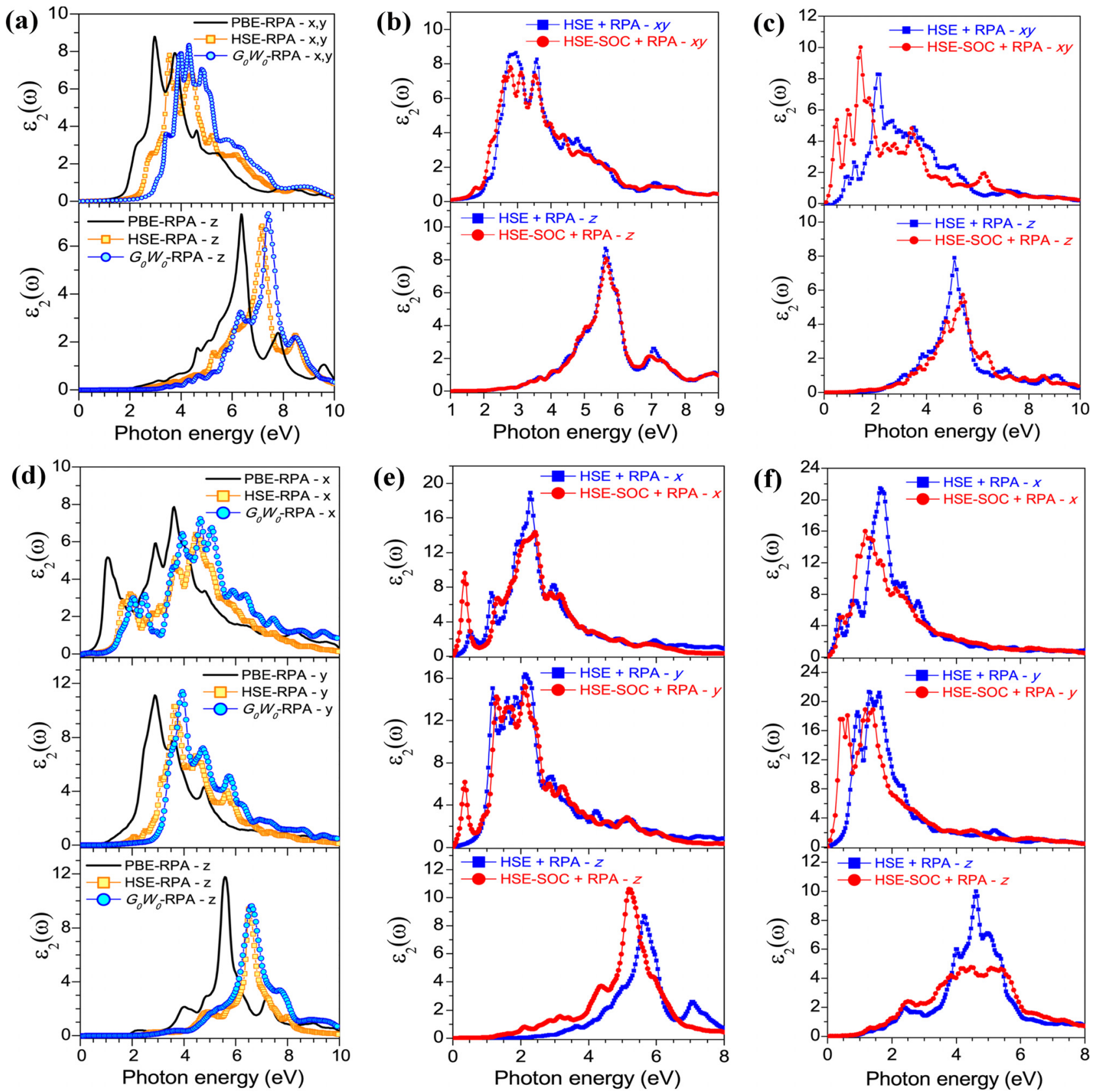

FIG. 5. Imaginary parts of the dielectric function versus photon energy for (top panel) $h b$ - and (bottom panel) swlaw-structures of As, Sb, and Bi monolayers (SL) for light polarized along the $x, y$, and $z$ directions. (a) and (d) Optical properties of single-layer and bilayer arsenene phases. Reproduced with permission from Kecik et al. Phys. Rev. B 94 205410 (2016). Copyright 2016 American Physical Society. (b), (c), (e), and (f) Structure dependent optoelectronic properties of monolayer antimonene, bismuthene, and their binary compounds. Reproduced with permission from Kecik et al. PhysChemChemPhys, in press (2019).

Although the band-edge of $h b$-Bi points to slightly above $0.5 \mathrm{eV}$, the significant peak is set in the visible, around $2.2 \mathrm{eV}$ (see Fig. 5). ${ }^{178}$ However, both the onset and luminescence peaks are redshifted by nearly $0.6 \mathrm{eV}$, when SOC effects are included, in compliance with the closing band-gap, once SOC is included to the HSE calculation-from 0.80 to $0.32 \mathrm{eV}$. $a w$ - $\mathrm{Bi}$ displays optical anisotropy, with an onset in the vicinity of 0 -limit, followed by a broadened optical activity range until nearly $5 \mathrm{eV}$ along the $x$-direction and relatively pronounced peaks are concentrated within $0.4-2 \mathrm{eV}$ along the $y$-direction. The optical response of $h b$-Bi is substantially affected when the electron-hole interactions are taken into account, with enhanced absorption even above the quasiparticle gap. A first exciton binding with a binding energy of $0.18 \mathrm{eV}$ is predicted (see Fig. 6). ${ }^{178}$ 

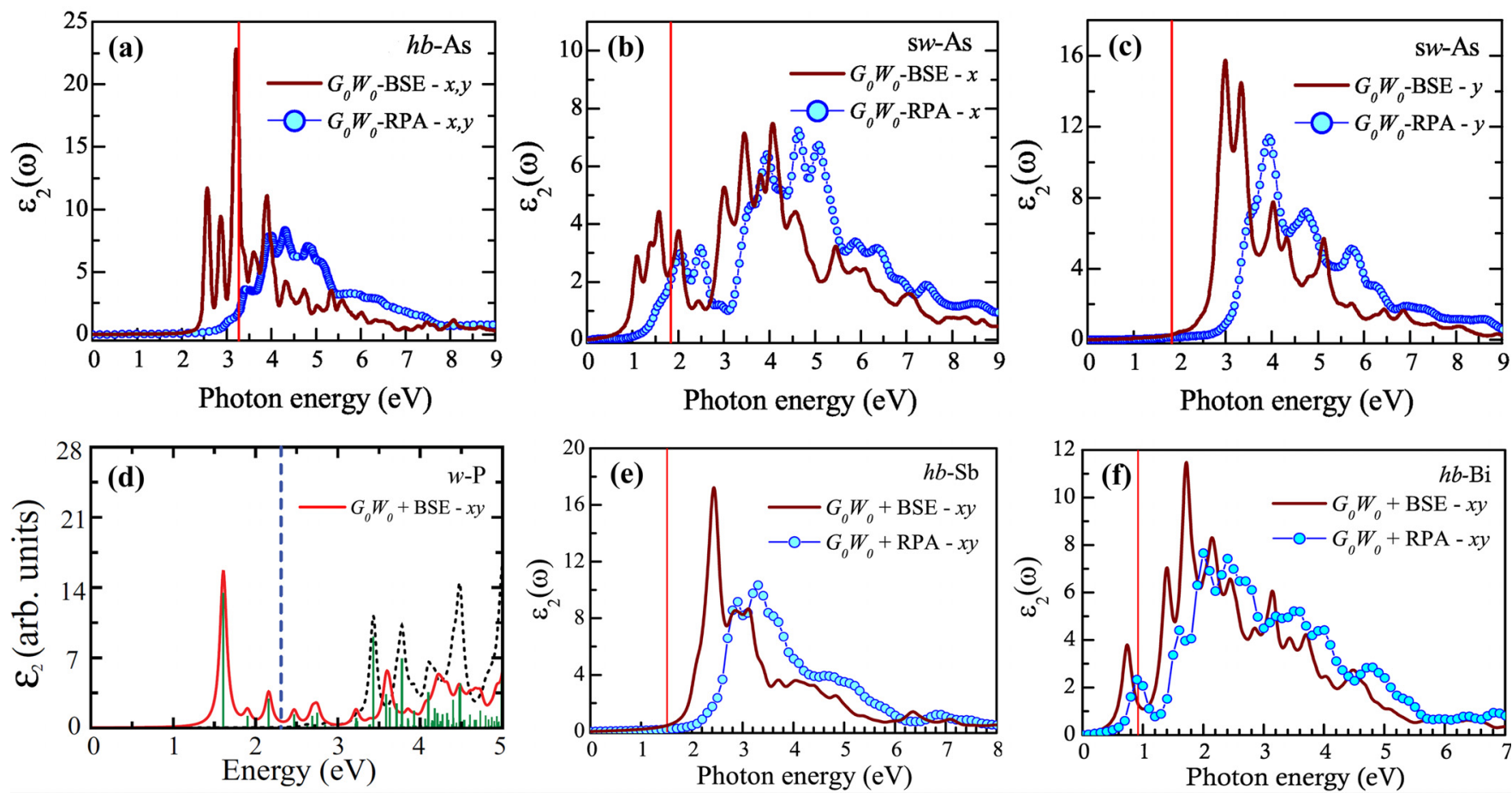

FIG. 6. Electron-hole effects taken into account by employing BSE for calculating the optical response of SL (a)-(c) $h b$-As and sw-As (Reproduced with permission from Kecik et al. Phys. Rev. B 94, 205410 (2016). Copyright 2016 American Physical Society), (d) sw-P (Reproduced with permission from Cakir et al. Phys. Rev. B 90, 205421 (2014). Copyright 2014 American Physical Society), (e) and (f) hb-Sb and hb-Bi phases (Reproduced with permission from. Kecik et al. Phys. Chem. Chem. Phys. in press (2019)).

In summary, the electronic bandgap, optical gap, and exciton binding energies can be tuned by polarizing light along different crystalline orientations. Also, strain and many-body effects are important for modifying the electronic and optical properties of most of the monolayers of group-VA elements. Furthermore, SOC effects impose different levels of alterations in the optoelectronic properties of especially pristine $\mathrm{Sb}$ and $\mathrm{Bi}$ monolayers.

\section{ADATOMS, ADMOLECULES, AND DEFECTS: FUNCTIONALIZATION}

The unusual properties attained through the adsorption of adatoms or induced defects of bare graphene and similar monolayers ${ }^{203-206}$ have led to a growing research interest in the functionalization of bare pnictogen monolayers. In this section, we review recent studies aiming at the modification of the properties of nitrogenene, phospherene, arsenene, antimonene, and bismuthene phases outlined above. Data related to the adsorption configurations, equilibrium adsorption sites, and corresponding binding energies are outlined in Fig. 7. Relevant data related to the physisorption and dissociation of molecules, $\mathrm{H}_{2}, \mathrm{O}_{2}$, and $\mathrm{H}_{2} \mathrm{O}$ on selected $h b$ - and $s w$ - (aw-) structures of group-VA monolayers are shown in Fig. 8.

\section{A. Nitrogene}

Since the synthesis of nitrogene continues to be a fundamental challenge, its functionalization has been studied only by theoretical methods. Adatoms such as $\mathrm{H}, \mathrm{Li}, \mathrm{O}, \mathrm{Al}, \mathrm{P}, \mathrm{Cl}, \mathrm{Ti}, \mathrm{As}$, and $\mathrm{Sb}$ adsorbed on the surface of $2 \mathrm{D}$ nitrogene can form strong bonds inducing minute or moderate local deformations of the substrate, leading to a diversity of the electronic and magnetic properties ${ }^{207}$ On the other hand, the adsorption of adatoms $\mathrm{B}, \mathrm{C}, \mathrm{N}$, and $\mathrm{Si}$ give rise to local reconstruction in nitrogene followed by bond breaking, even dissociation of the host $\mathrm{N}$ atoms. While molecules such as $\mathrm{H}_{2}, \mathrm{O}_{2}, \mathrm{H}_{2} \mathrm{O}$, and $\mathrm{N}_{2}$, interact weakly with nitrogene, molecules like $\mathrm{H}_{2}$ and $\mathrm{O}_{2}$ dissociate at the edges of a nitrogene flake and form $\mathrm{NH}_{2}$ and $\mathrm{NO}_{2}$ molecules, respectively. Single vacancy in nitrogene attains a permanent magnetic moment through the uncompensated spins of the missing nitrogen atom. Vacancy gives rise to spin-polarized states in the fundamental bandgap which are localized at the vacancy site, in particular at $\mathrm{N}$ atoms surrounding the vacancy. Single vacancy in nitrogene is found to be stable at $0 \mathrm{~K}$, nevertheless it is prone to severe deformations under thermal excitations at $300 \mathrm{~K}$. Similar calculations resulted in also a structural instability of the divacancy in nitrogene ${ }^{207}$

\section{B. Phosphorene}

Kulish et al. ${ }^{208}$ have investigated the electronic and magnetic properties of 20 different adatoms ( $\mathrm{Li}, \mathrm{Na}, \mathrm{K}, \mathrm{Cu}, \mathrm{Ag}, \mathrm{Au}, \mathrm{Pd}, \mathrm{Pt}, \mathrm{Ti}, \mathrm{V}$, $\mathrm{Cr}, \mathrm{Mn}, \mathrm{Fe}, \mathrm{Co}, \mathrm{Ni}, \mathrm{Si}, \mathrm{Ge}, \mathrm{P}, \mathrm{H}$, and O) adsorbed on $s w-P$ phospherene. This theoretical study showed that the adatoms form strong bonds with chemisorption energies twice of those on graphene. As usual, alkali adatoms ( $\mathrm{Li}, \mathrm{Na}$, and $\mathrm{K}$ ) donate a large amount of charge to phosphorene, hence they can be utilized for n-type doping, whereas $\mathrm{Au}$ and $\mathrm{Pt}$ adatoms act as electron acceptors, suggesting that $\mathrm{Au}$ or Pt decoration can be used to produce p-type doping of phosphorene. ${ }^{208}$ As for light non-metallic atom substitution in $h b$-P, atoms like B, C, 
$\mathrm{N}, \mathrm{O}$, and $\mathrm{F}$ formed strong bonds with $\mathrm{P}$ atom by retaining the semiconducting behavior of the substrate. ${ }^{209}$ However, it is predicted that the electronic properties of $h b-\mathrm{P}$ can be effectively tuned by $\mathrm{Al}$ and $\mathrm{Sb}$ substitution leading to indirect-to-direct-gap transition. ${ }^{210}$ In other studies, the magnetism of phosphorene phases has been investigated actively. It was shown that nonmagnetic pristine $s w$-P phosphorene becomes magnetic after the adsorption of $\mathrm{N}, \mathrm{Fe}$, or Co adatoms. ${ }^{211}$ Neither $\mathrm{P}$ vacancy nor strain alone induces magnetism in phosphorene. ${ }^{212}$ However, when biaxial strain or uniaxial strain along the zigzag direction reached critical values, $s w$ - $\mathrm{P}$ containing $\mathrm{P}$ vacancies attained magnetic moment of $\sim 1 \mu_{B}$ per vacancy site. This is due to spinpolarized $p$-orbitals of low coordinated $\mathrm{P}$ atoms next to the vacancy. ${ }^{213}$ Additionally, vacancies induce local stress concentration and cause early bond-breaking. It is shown that the Young's modulus decreases with increasing defect concentration. ${ }^{214} \mathrm{Yu}$ et al. ${ }^{212}$ examined the substitution of $3 d$-TM impurities ( $\mathrm{Sc}, \mathrm{Ti}, \mathrm{V}, \mathrm{Cr}, \mathrm{Mn}, \mathrm{Fe}, \mathrm{Co}, \mathrm{Ni}$ ) in black and blue phosphorene in order to investigate their dilute magnetic characters and half-metallic properties. For black phosphorene, the Ti, V-, Cr-, Mn-, Fe-, and Ni-doped systems have dilute magnetic semiconductor properties, while Sc- and Co-doped systems have no magnetism. For $h b$-phosphorene, the Ni-doped system shows half-metallic properties, while V-, Cr-, Mn-, and Fe-doped systems show dilute magnetic semiconductor characters, Sc- and Co-doped systems show non-magnetism.

The weak interaction between phosphorene and the $\mathrm{O}_{2}$ or $\mathrm{H}_{2}$ molecule has been treated in various studies. ${ }^{215-220}$ Several acidic gases $\left(\mathrm{CO}_{2}, \mathrm{NO}_{2}\right.$, and $\left.\mathrm{SO}_{2}\right)$ are readily adsorbed on $\mathrm{Li}$ and $\mathrm{Al}$ decorated phosphorene. $\mathrm{NO}_{2}$ and $\mathrm{SO}_{2}$ can bind strongly on $\mathrm{Al}$ decorated phosphorene, while these gases can dissociate on Pt-decorated phosphorene. ${ }^{221}$ The coadsorption of two foreign atoms (such as $\mathrm{S}, \mathrm{Si}$, or $\mathrm{Al}$ ) to phosphorene is more stable than that of the corresponding single atom adsorption. ${ }^{222}$ Likewise, co-doping of transition metal atom, vanadium $(\mathrm{V})$ and nonmetallic atoms $\mathrm{B}, \mathrm{C}, \mathrm{N}, \mathrm{O}$ (such as $\mathrm{V}-\mathrm{B}, \mathrm{V}-\mathrm{C}$, $\mathrm{V}-\mathrm{N}, \mathrm{V}-\mathrm{O}$ ) on phosphorene reduces the bandgap. Moreover, the optical absorption edges of these systems shift towards lower energies, generating the redshift phenomenon. ${ }^{223}$

\section{Arsenene}

Chemisorption of various adatoms $(\mathrm{H}, \mathrm{Li}, \mathrm{B}, \mathrm{C}, \mathrm{N}, \mathrm{O}, \mathrm{Al}, \mathrm{Si}, \mathrm{P}$, $\mathrm{Cl}, \mathrm{Ti}, \mathrm{Ga}, \mathrm{Ge}, \mathrm{As}, \mathrm{Se}$, and $\mathrm{Sb}$ ) and physisoption of selected molecules $\left(\mathrm{H}_{2}, \mathrm{O}_{2}\right.$, and $\left.\mathrm{H}_{2} \mathrm{O}\right)$ on the arsenene monolayer have been previously studied. ${ }^{110}$ It was reported that the binding energies of single adatoms range from 1.03 to $4.58 \mathrm{eV}$; the majority of them give rise to localized states in the fundamental bandgap. Moreover, $\mathrm{P}$ and Ti adatoms can attribute half metallic character to the system. When arsenene is substituted by $\mathrm{N}$ or $\mathrm{P}$ atoms, its bandgap is widened, which is in contrast to the case of the substitution by $\mathrm{Sb}$ and $\mathrm{Bi}$ atoms. ${ }^{224} \mathrm{H}_{2}, \mathrm{O}_{2}$, and $\mathrm{H}_{2} \mathrm{O}$ molecules neither form strong chemical bonds with the arsenene monolayer nor dissociate; they are physisorbed with a weak binding energy. ${ }^{110,224}$ However, $\mathrm{H}_{2}$ and $\mathrm{O}_{2}$ molecules strongly interact at the edges of arsenene flakes and are subsequently dissociated into constituent atoms. ${ }^{110} \mathrm{NO}_{2}$ and $\mathrm{SO}_{2}$ can be bound on $\mathrm{B}$ doped arsenene, suggesting an efficient application in metal-free catalysts. ${ }^{225} \mathrm{~A}$ similar behavior was also observed for Ge substitution. It was reported that $\mathrm{NH}_{3}$ and $\mathrm{NO}_{2}$, which are weakly bound on bare arsenene, can readily be adsorbed to Ge-substituted arsenene. ${ }^{226}$ $h b$-As doped with transition metals (Ti, V, Cr, Mn, Fe, and Ni) maintained the stability at room temperature and attained magnetization. While Sc and Cr doped arsenene retained its semiconducting character, $\mathrm{Ti}$ and $\mathrm{V}$ doped arsenene transformed to metal. More importantly, Fermi level crosses one of the spin-up or spin-down channels in Ti, V, Mn, and Fe doped arsenene, which causes a halfmetallicity for these systems. ${ }^{227}$ However, in another study it was predicted that while a half-metallic state occurs for arsenene doped with $\mathrm{Ti}$ and Mn, a spin-polarized semiconducting state forms when arsenene doped with $\mathrm{V}, \mathrm{Cr}$, and $\mathrm{Fe}{ }^{228}$ Two $\mathrm{Mn}$ atoms or two $\mathrm{Fe}$ atoms adsorbed to $h b$-As systems have antiferromagnetic states. ${ }^{229}$

Formation of point defects, namely, single vacancy, divacancy, and Stone-Wales (SW) defects, was considered for $h b$-As and $s w$ As. ${ }^{230,231}$ SW defects were found to be thermodynamically more favorable than single vacancy and divacancy and were predicted to be stable at room temperature. Topological defects in arsenene significantly alter the optical spectra. The charge distribution calculations predicted that the charge specific states are localized around the vacancy. ${ }^{230}$ Single vacancy defects were found to significantly modify the electronic properties by introducing defect levels (localized states) within the gap. The band structures of divacancy defects in $s w$-As were predicted to be less modified as compared to $h b$-As, most likely due to the better accommodation of the defect induced strain. It was shown that vacancies in $h b$-As lead to a direct bandgap semiconductor. ${ }^{231}$ Recently, it was reported that some nanohole types induce magnetic features and reduce the bandgap of arsenene, while other types of nanoholes exhibit nonmagnetism and large bandgap. ${ }^{232}$

\section{Antimonene}

Single adatom (H, Li, B, C, N, O, Al, In, Si, P, Cl, Ti, As, and Sb) adsorption unveils significant binding energy on antimonene. Some of these adatoms are implemented into the antimonene crystal through local reconstructions, such as the SW defect. Most of these adatoms have localized states near the band edges and attribute crucial electronic properties to antimonene monolayers. Some adatoms give rise to spin polarization and attain magnetic moments. ${ }^{233}$ The results show that the atmospheric gas molecules $\left(\mathrm{N}_{2}, \mathrm{CO}_{2}, \mathrm{O}_{2}\right.$, and $\left.\mathrm{H}_{2}\right)$ are weakly bound to antimonene, while the polluted gas adsorbates $\left(\mathrm{NH}_{3}\right.$, $\mathrm{SO}_{2}, \mathrm{NO}$, and $\mathrm{NO}_{2}$ ) show relatively strong affinity toward antimonene with considerable adsorption energies and significant charge transfers. ${ }^{234}$ Also, $\mathrm{NO}, \mathrm{NO}_{2}, \mathrm{H}_{2} \mathrm{O}, \mathrm{O}_{2}$, and $\mathrm{NH}_{3}$ molecules act as a acceptor in antimonene, whereas $\mathrm{H}_{2}$ behaves as a donor. Since $\mathrm{O}_{2}$ molecule has a low barrier for dissociation on antimonene, the oxidation in the course applications can take place easily. ${ }^{235}$

While $\mathrm{CO}$ is physisorbed on pristine antimonene, it forms chemical bonds with adatoms $\mathrm{Al}, \mathrm{Cr}, \mathrm{Fe}, \mathrm{Co}, \mathrm{Ni}, \mathrm{Cu}, \mathrm{Pd}$, and $\mathrm{Si}$, which are already adsorbed to antimonene. An external electric field ranging from $0.21 \mathrm{eV} / \AA$ to $0.5 \mathrm{eV} / \AA$ can improve $\mathrm{CO}$ gas sensitivity of antimonene. ${ }^{236}$ In another study, it is reported that adsorption of organic molecules, such as tetrathiafulvalene (TTF) and tetracyanoquinodimethane (TCNQ) can be used as electron and hole dopants to attain nand p-type antimonene semiconductors. In this way, the bandgap is reduced with deep donor (TTF) and shallow acceptor (TCNQ) states, respectively. Moreover, the co-adsorption of TTF and TCNQ on antimonene can attain an n-type semiconductor with shallow donor states. $^{237}$ 
Antimonene defected with monovacancy was found to be metallic, whereas antimonene defected with divacancy preserved its original semiconducting character with a narrower bandgap. ${ }^{238}$ Formation energies of these defects are smaller than those in graphene and silicene. $^{239}$

\section{E. Bismuthene}

Interaction of the adatoms, $\mathrm{H}, \mathrm{C}, \mathrm{O}, \mathrm{Si}, \mathrm{P}, \mathrm{Ge}, \mathrm{As}, \mathrm{Se}, \mathrm{Sb}, \mathrm{Pb}, \mathrm{Sn}$, and $\mathrm{Te}$ with $h b$-Bi and $a w$-Bi bismuthene phases has been investigated using DFT calculations. ${ }^{132}$ They found that the binding energies are high (ranging from $1.1 \mathrm{eV}$ to $4.1 \mathrm{eV}$ ) and are dominated by chemical bonding through charge exchange between the adatoms and nearest host $\mathrm{Bi}$ atoms. The vdW contribution to the binding energies is generally small except for the bonding of $\mathrm{Si}$ and $\mathrm{Sb}$ to $h b$ - $\mathrm{Bi}$. Adatoms give rise to localized gap states at the specific region of the band structure for $s w$-Bi. Single vacancy as a point defect has small formation energy and hence can form readily at room temperature. Bismuth atoms surrounding the vacancy have $s p^{2}$ type dangling bonds which make vacancy a chemically active site in bismuthene. Notably, the formation energy of divacancy is unexpectedly small owing to the rebonding of $\mathrm{Bi}$ atoms surrounding the divacancy. Moreover, they found that pristine $\mathrm{SL} h b-\mathrm{Bi}$ is a nontrivial topological insulator which can be changed by the adsorption of selected adatoms and creation of single vacancies. On the other hand, they reported that the nontrivial band topology disappeared upon the creation of a single vacancy in each supercell periodically, since $h b$ - $\mathrm{Bi}$ is metallized by the bands derived from the localized states of an isolated single vacancy. In contrast, the nontrivial band topology is maintained in the divacancy periodically repeated in the same supercell, since the bands derived from defect states are removed from the bandgap through rebondings of $\mathrm{Bi}$ atoms surrounding the divacancy. ${ }^{132}$

\section{F. Hydrogenation and halogenation}

Pnictogen monolayers decorated by hydrogen or halogen acquire novel properties, and hence deserve a particular attention. For example, while monolayer black phosphorene has a direct bandgap value of $\sim 1.5 \mathrm{eV},{ }^{243}$ this will be increased to $2.27 \mathrm{eV}$ with a direct-indirect transition when it is fully fluorinated. ${ }^{244}$ Even if phosphorene monolayers could gain instability for full coverage of hydrogen or oxygen, ${ }^{244}$ few other studies contradict with these conclusions. ${ }^{218}$ In addition to bandgap tuning, controlled chemisorption of fluor or oxygen atoms on $s w$-P can change the unique anisotropic carrier effective mass for both the electrons and holes. ${ }^{245}$ Even though $h b$-P has a larger bandgap than $a w-\mathrm{P}$, upon hydrogenation, the former attains the Dirac cone at the K-point. ${ }^{246}$ Recently, Sun et al. ${ }^{91}$ predicted that halogenated phosphorenes having formula units $\mathrm{P}_{2} \mathrm{~F}_{2}, \mathrm{P}_{2} \mathrm{Cl}_{2}, \mathrm{P}_{2} \mathrm{Br}_{2}$, and $\mathrm{P}_{2} \mathrm{I}_{2}$ also display Dirac cones at the K-point in their band structures. Not only $h b$-P, but also $h b$-As can have X-type Dirac cone at the K-point. Zhang et al. showed that fully hydrogenated arsenene is a nonmagnetic planar Dirac material, while semi-hydrogenated arsenene has magnetization $0.92 \mu_{B}$ due to unsaturated $p_{z}$ orbitals. ${ }^{247}$ Later, Wang et al. ${ }^{248}$ proved the dynamical stability of fully hydrogenated arsenene and showed that the spin-orbit coupling opens a gap of $193 \mathrm{meV}$ at the Dirac cone. Dynamical stability and the formation of Dirac cone after full halogenation of arsenene were also extensively studied theoretically. ${ }^{249-252}$ The latest study predicted that decoration of arsenene with methyl $\left(\mathrm{CH}_{3}\right)$ and hydroxyl $(\mathrm{OH})$ groups are dynamically stable and have nontrivial bandgaps $\left(0.184 \mathrm{eV}\right.$ for $\mathrm{AsCH}_{3}$ and $0.304 \mathrm{eV}$ for $\mathrm{AsOH}$ ) in the system. ${ }^{253}$ Song et al. predicted by first-principles calculations that $\mathrm{SbX}$ and $\mathrm{BiX}(\mathrm{X}=\mathrm{H}, \mathrm{F}, \mathrm{Cl}$, and $\mathrm{Br})$ monolayers remain stable at even $600 \mathrm{~K}$. According to their extensive analysis, SbX and BiX structures have large bulk gap values from 0.32 to $1.08 \mathrm{eV}$ with SOC effects. ${ }^{254}$ Stability of SbX monolayers are demonstrated by Zhang et al. who calculated their phonon structures. ${ }^{255}$ Low coverage of halogens (like coverage values $1 / 8$ and $1 / 18$ ) on the $\mathrm{Sb}$ monolayer can induce magnetization or convert the system from a semiconductor to a metal. ${ }^{256}$ It is also predicted that antimonene fully covered by oxygen behaves as a topological insulator with a bandgap of $177 \mathrm{meV}$ at the $\Gamma$-point.

\section{NANORIBBONS}

Earlier studies on the nanoribbons of 2D monolayers showed that their electronic properties largely depend on their edge geometry (armchair or zigzag edge), as well as their widths, see Fig. 9. For example, while pristine phosphorene has a direct bandgap of $\sim 1.5 \mathrm{eV}$, its armchair nanoribbons are indirect bandgap semiconductors, whereas its zigzag nanoribbons become metallic; ${ }^{243}$ even though both types of nanoribbons exhibit semiconducting properties when their edges are passivated by hydrogen. ${ }^{258-261}$ Generally, bandgaps of armchair nanoribbons show an oscillatory variation as a function of the width, where the bandgap of $s w$-P armchair nanoribbons (APNRs) decreases monotonically with the increasing ribbon width. ${ }^{258,262}$ This facilitates APNRs being more promising than those of $\mathrm{MoS}_{2}$ and graphene for FET applications. Both armchair and zigzag (ZPNR) nanoribbons of $s w$-P have positive formation energies, which suggests that their experimental synthesis is fully achievable. ${ }^{258,262}$ Sarvari et al. ${ }^{263}$ showed that the performance of APNR-FET changes significantly depending on the number of phosphorene layers and length of the device channel. For example, they showed that the bandgap of the material is reduced by increasing the number of APNR layers. Furthermore, the ON/OFF current ratio for mono-, bi-, and trilayer APNR-FETs increases with the increasing of the channel length. ${ }^{263}$ The applied electric field tunes the bandgap of phospherene nanoribbon, which varies linearly for large electric fields, yet tends to be quadratic for small fields. ${ }^{264}$

Recently, multilayer arsenene and antimonene nanoribbons were synthesized using the plasma assisted process on InAs and InSb substrates, respectively. ${ }^{66,265}$ Their bandgaps were estimated by the photoluminescence (PL) measurements and were found as $2.3 \mathrm{eV}$ for arsenene nanoribbons and $2.03 \mathrm{eV}$ for antimonene nanoribbons. These bandgaps are very suitable for transistor and LED applications. Ab-initio calculations show that similar to phosphorene, bandgaps of hydrogenated arsenene and antimonene nanoribbons have semiconducting properties, and their bandgaps decrease monotonically with the increasing width. ${ }^{266-268}$ Both arsenene and antimonene armchair nanoribbons have indirect bandgaps, while their zigzag nanoribbons have direct bandgaps independent of the ribbon width. ${ }^{267}$ However, Zhang et al., ${ }^{.1}$ found that indirectdirect bandgap transition or vice-versa occurs by modifying the ribbon width in both armchair and zigzag arsenene nanoribbons. This kind of transition was also observed under the tensile strain for arsenene nanoribbons. ${ }^{266}$ Song et al. ${ }^{269}$ predicted that zigzag nanoribbons of $h b$ - and $a w$-Sb have magnetic moments at the edge atoms in the range of -0.10 to $0.08 \mathrm{~m} \mu_{B}$. Similar results were 


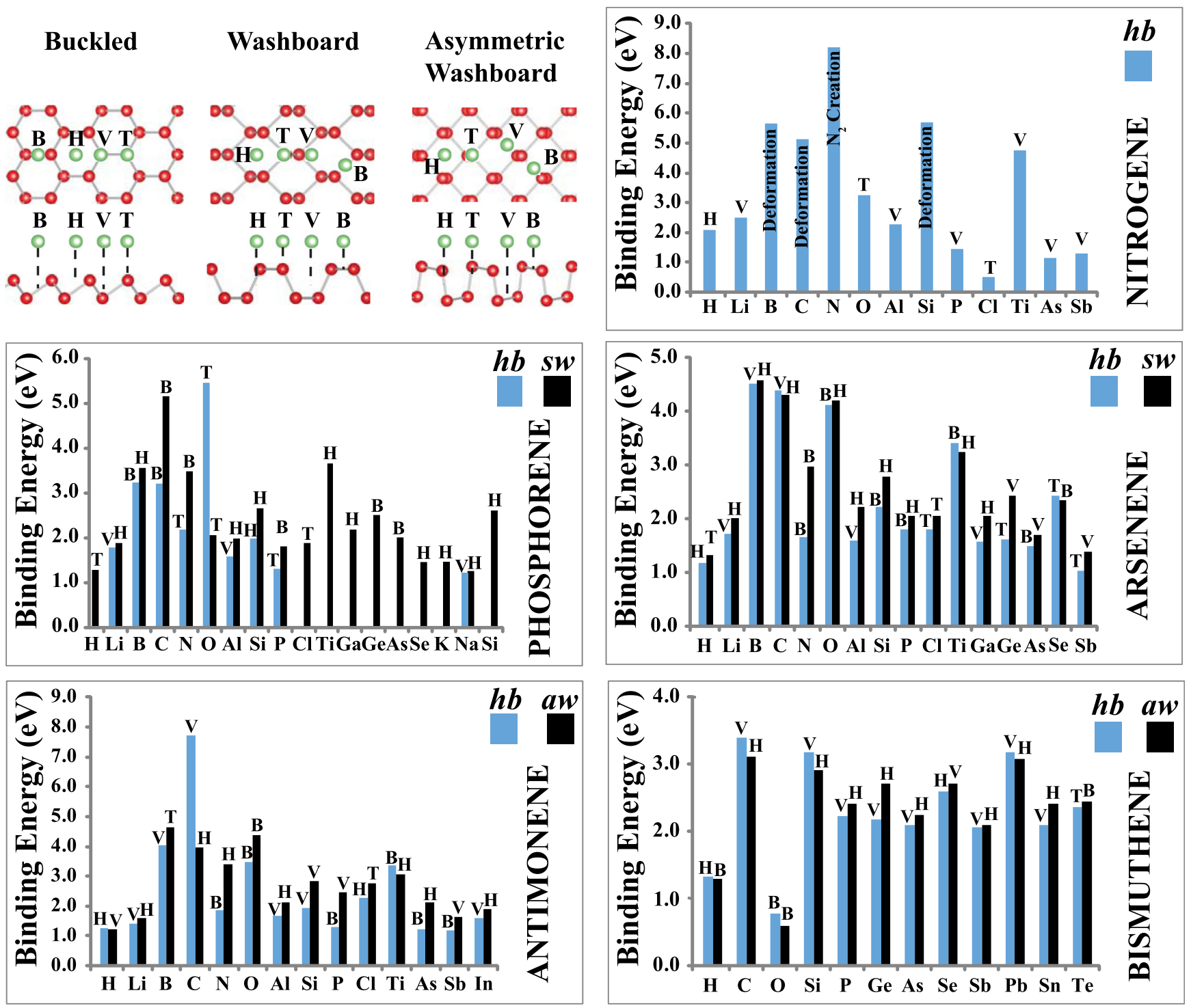

FIG. 7. Top-left panel shows the top and side views of the possible adsorption sites on the $h b$-structure: Top site (T) is where the adatom is on top of the host group-VA atoms. The bridge site is denoted by $(\mathrm{B})$, where the adatom is above the center of the $\mathrm{X}-\mathrm{X}$ bond. $(\mathrm{H})$ stands for the hollow site, where the adatom is located above the center of hexagons. The valley site is denoted by $(\mathrm{V})$, where the adatom is placed on top the low-buckled host atom. Similar adsorption sites are also indicated for $s w$ - and aw-structures. Other panels show the calculated binding energies of adatoms adsorbed at different equilibrium sites of $h b$-, sw- or aw-phases of nitrogene, phosphorene, ${ }^{240-242}$ arsenene, antimonene, and bismuthene, respectively. Reproduced with permissions from Ersan et al., J. Phys. Chem. C 120, 14345-14355 (2016). Copyright 2016 American Chemical Society; Kadioglu et al., Phys. Rev. B 96, 245424 (2017). Copyright 2017 American Physical Society; Kadioglu et al., J. Phys. Chem. C 121, $6329-6338$ (2017). Copyright 2017 American Chemical Society; Ding and Wang J. Phys. Chem. C 119, 10610-10622 (2015). Copyright 2015 American Chemical Society; Zhu et al., Phys. Status Solidi B 253, 1156-1166 (2016). Copyright 2016 John Wiley and Sons.

predicted for the zigzag arsenene nanoribbons $\left(0.07 \mu_{B}\right)^{270}$ Aktürk et al. performed the optimization calculations using the $2 \times 1$ unit cell of $h b$-Sb nanoribbons in order to see the reconstruction of the edge atoms, where ferromagnetic features were detected. ${ }^{46}$ They calculated $0.87 \mathrm{eV}$ energy gap between the filled spin-up and empty spin-down bands, from HSE calculation. Similar edge magnetism, however, was not observed in the armchair $\mathrm{Sb}$ nanoribbons. ${ }^{46}$
Giant magnetoresistance can be traced in armchair arsenene nanoribbons, combining the suitable ribbon width and magnetic state. ${ }^{271,272}$ Abid et al. ${ }^{273}$ showed that the edge magnetism of zigzag arsenene and antimonene nanoribbons disappear with $\mathrm{H}$ saturation, contrarily restored when saturated by $\mathrm{O}$ atoms. Although several experimental studies on bismuth nanoribbons exist, only few theoretical investigations are present. Experimental studies revealed that, bismuthene nanoribbons have large linear 


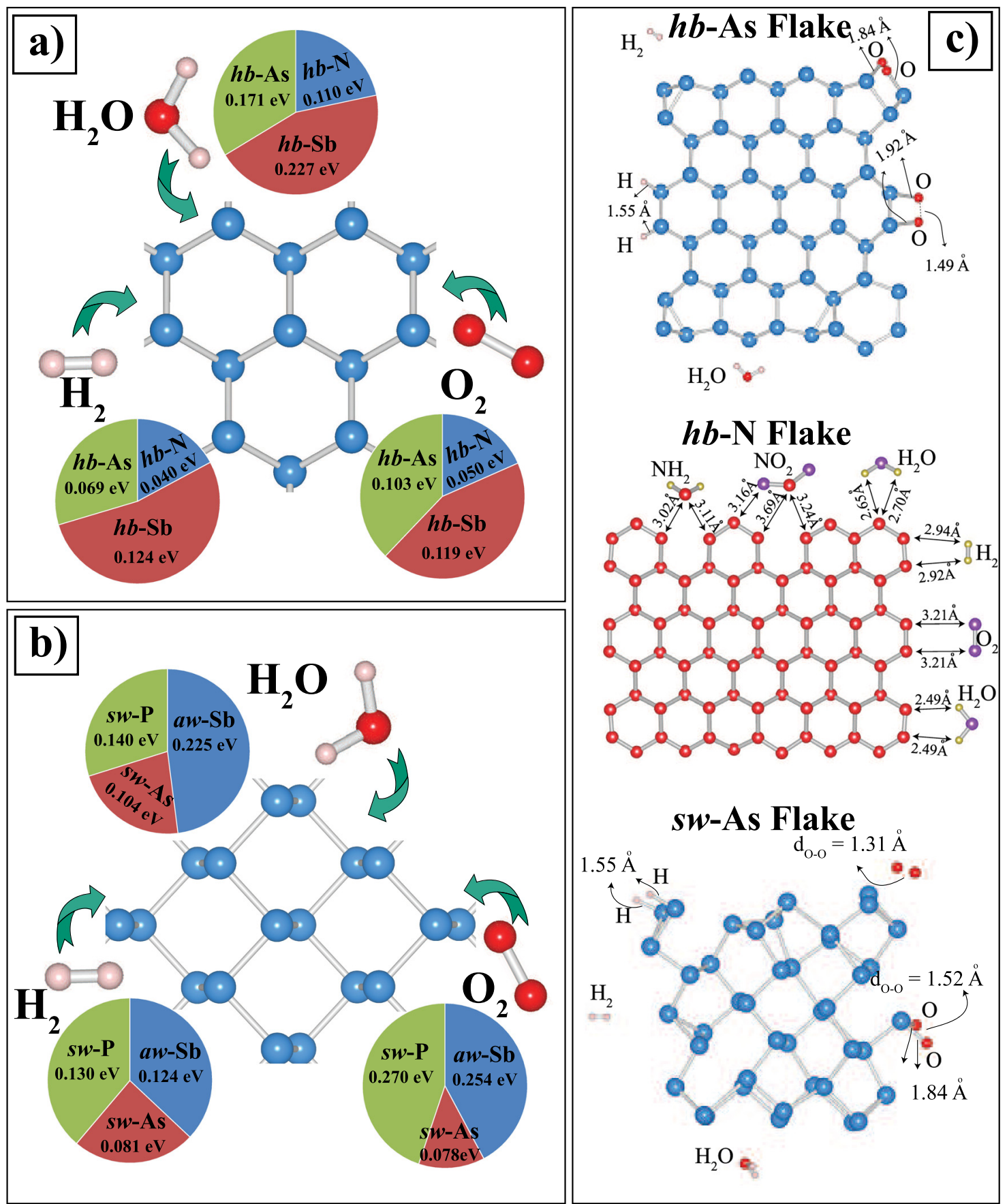

FIG. 8. (a) Physisorption energies of $\mathrm{H}_{2}, \mathrm{O}_{2}$, and $\mathrm{H}_{2} \mathrm{O}$ on $h b$-structures of group-VA monolayers. (b) The same for sw- or aw-structures. (c) Atomic configuration and structural parameters for the dissociative adsorption and molecular physisorption of selected molecules in $h b$-As, $h b-\mathrm{N}$, and $s w$-As. Reproduced with permissions from Ersan et al., J. Phys. Chem. $\mathrm{C}$ 120, 14345-14355 (2016). Copyright 2016 American Chemical Society; Kadioglu et al., J. Phys. Chem. C 121, 6329-6338 (2017). Copyright 2017 American Chemical Society.

magnetoresistance, ${ }^{274}$ and they are robust to the oxidation. ${ }^{275,276}$ Sun et al. ${ }^{69}$ present experimental and theoretical results, with a very large energy gap of $0.4 \mathrm{eV}$ detected in the middle of $\mathrm{Bi}(110)$ nanoribbons. Recently, Wang et al. ${ }^{277}$ showed that armchair nanoribbons of bismuthene display topologically nontrivial features. Yao et al. investigated nanoribbons of so-structures and predicted ferromagnetic semiconductor properties for P, As, and $\mathrm{Sb}$. Due to the reconstruction on the edges and dangling bonds, this rearrangement led to a Dirac point near $\pi$ in the band structure of the nitrogene so-nanoribbon. ${ }^{72}$ 


\section{BILAYER AND MULTILAYERS}

Bilayers and multilayers of 2D structures have attracted interest as alternative materials for tuning the electronic and optical properties. In addition to the number of layers, the stacking sequence of single layers is another critical parameter. In Figs. 10 and 11, the stacking sequences of different bilayer phases and the variation of their interlayer interaction energies are depicted.

Research on nitrogene multilayers is limited with only a few studies. Despite the very strong $\mathrm{N}_{2}$ molecule, theoretical studies predicted that the monolayer of nitrogene in the buckled hexagonal structure can be dynamically and thermally stable. ${ }^{44}$ Furthermore, it is found that bilayer nitrogene attained minimum energy for the $\mathrm{AB}$ stacking sequence, and similarly $\mathrm{ABA}$ stacking sequence is favorable for trilayer nitrogene. ${ }^{44}$ Owing to the weak interlayer interactions, the band structure is not affected significantly in the bilayer form and slightly modified in multilayer forms. ${ }^{44}$ The bandgap decreases with increasing number of layers. ${ }^{278}$

The bandgap of few-layer black phosphorene decreases with the increasing number of layers. ${ }^{243,279}$ Additionally, high carrier mobility, and high transport anisotropy in black phosphorene can be tuned by layer thickness. ${ }^{243}$ It is reported that the drain current and mobility in black phospherene FETs can change with the sample thickness. ${ }^{280}$ Adsorption and optical conductivity increase with the decreasing thickness. ${ }^{281} \mathrm{AA}, \mathrm{AB}$, and $\mathrm{AC}$ stacked bilayer phosphorenes have been found to have direct bandgaps of $0.97,1.04$, and $0.78 \mathrm{eV}$, respectively, and these bandgaps can be tuned by the applied electric field. The investigation of the optical absorption spectra as a function of stacking sequence suggested that phosphorene bilayers can serve for solar energy applications. ${ }^{282}$ It is also shown that topological behavior in phosphorene bilayer depends on the stacking order and the optical absorption spectrum of bilayer phosphorene extends to the far infrared region in the quantum spin Hall state. ${ }^{283}$

As for arsenene, while the fundamental bandgap is indirect in monolayer, it changes to the direct bandgap in multilayers. ${ }^{284,285}$ Carrier mobility is predicted to increase monotonically as the number of layers increase. This plays a crucial role in device performance. ${ }^{284}$ AA stacking order was found to be energetically more favorable than $\mathrm{AB}$ stacking in bilayer of $h b$-arsenene. ${ }^{50,286}$ Also, AAA stacking was shown to be favorable than ABA stacking type in trilayer for both hexagonal buckled and symmetric washboard structures. Although various studies reported about energetically different favorable stacking type of washboard bilayer arsenene, a recent study which associates DFT with QMC (quantum monte carlo) calculations pointed to the $\mathrm{AB}$ stacking order as the most stable structure. ${ }^{286}$ The bandgap decreases in the buckled trilayer, and closed in the washboard trilayer. ${ }^{50} \mathrm{Mi}$ et al. have studied especially the effects of stacking sequences of arsenene bilayers and have concluded that $\mathrm{AB}$ stacking shows indirect-direct transition while AA stacking leads to an indirect bandgap. ${ }^{287}$

Few-layer antimonene was isolated by liquid-phase exfoliation under ambient conditions. ${ }^{288}$ It was found that the lattice constant decreases with increasing thickness. Raman activity decreases by about three orders of magnitude from bulk to monolayer antimonene. ${ }^{288}$ Bilayer antimonene was found topologically trivial due to its intrinsically weak SOC. ${ }^{289}$ AA and AC stacking types of bilayer arsenene result in a nonzero bandgap, which are different from the $\mathrm{AB}$ stacking order, where especially AC stacking bilayer antimonene required an optimum absorption range for thermophotovoltaic cells. ${ }^{290}$

The electronic bandgap of bismuthene is also tunable by the number of layers. ${ }^{194}$ While monolayer $\mathrm{Bi}$ has a bandgap of around $0.50 \mathrm{eV}$, it becomes narrower and eventually closes as the number of layers increase up to six. ${ }^{291}$ Bilayer bismuthene maintains topological insulator behavior for $\mathrm{AB}$ stacking; however, it becomes a topological semimetal for the AA stacking sequence. ${ }^{289}$

\section{SUBSTRATE EFFECTS}

The effects of substrates on 2D group-VA monolayers will be reviewed concisely in this section, focusing on their vertical heterostructures with diverse substrates. This study was cited in Fig. 11(e) (see Ref. 292). Recently, several theoretical and experimental efforts were focused on vdW heterostructures such as phosphorene/TMD (Transition metal dichalcogenide), ${ }^{293}$ phosphorene/graphene, metal contacts to phosphorene such as $\mathrm{Cu}(111), \mathrm{Zn}(0001), \operatorname{In}(110), \mathrm{Ta}(110)$, and $\mathrm{Nb}(100)$ surfaces, ${ }^{294}$ defected hBN/black phosphorene heterostructures ${ }^{295}$ Heterostructures discussed in the text are shown in Fig. 12.

In the vertical heterostructure formed by graphene and phosphorene, the Schottky barrier height can be controlled by the perpendicular electric field and doping of phosphorene. ${ }^{296}$ In another study by $\mathrm{Hu}$ et al., ${ }^{297}$ once the interfacial distance was decreased between graphene and phosphorene, the Dirac point of graphene was moved from the conduction to the valance band of phosphorene, besides changing to a p-type Schottky contact. Graphene and hBN supported phosphorene heterostructures were studied by Davletshin et al., ${ }^{298}$ where graphene supported phosphorene was found metallic, and $\mathrm{BN}$ phosphorene as a semiconductor with around $1 \mathrm{eV}$ bandgap. However, under in-plane uniaxial strain, the graphene supported phospherene can open a bandgap. ${ }^{299}$ Fabricated h-BN/Phosphorene/ h-BN transistors can be stable at ambient conditions for more than $300 \mathrm{~h}$, and exhibit ambipolar behavior. They display a gate-dependent metal-insulator transition, and their mobility can be up to $4000 \mathrm{~cm}^{2}$ $\mathrm{V}^{-1} \mathrm{~s}^{-1} \cdot{ }^{300}$ Single-layer and bilayer heterostructures of $s w$-P with graphene were studied and it was found that both the properties of graphene and phosphorene were preserved upon their contact. It is possible to tune the band structure of phosphorene relative to that of graphene through the application of an external electric field perpendicular to the system. This allows significant control of the Schottky barrier height, as well as the doping of phosphorene. ${ }^{301}$ The strong exciton binding energy of $s w$-P/TMD heterostructures indicates that their type-II heterostructures are desirable for excitonic solar cell applications. However, the calculated power conversion efficiency of the excitonic solar cells can be as high as $12 \%$ when phosphorene is paired with $\mathrm{ZrS}_{2}$ or $\mathrm{MoTe}_{2} .{ }^{302}$ It has been expressed that $h b-\mathrm{P} / \mathrm{MS}_{2}(\mathrm{M}=\mathrm{Nb}$, $\mathrm{Ta}$ ) heterostructures possess good structural stability with negative formation energy, enhanced electrical conductivity, improved mechanical flexibility (ultimate strain $>17 \%$ ) and high lithium-ion capacity $\left(528.257 \mathrm{mAh} \mathrm{g}^{-1}\right.$ for $h b$-P/NbS 2$) .{ }^{303}$ Zeng et al. showed that both $s w$ $\mathrm{P}$ and $h b$-P monolayers can be readily stabilized on $\mathrm{Cu}(111), \mathrm{Au}(111)$, and $\mathrm{GaN}(001)$ substrates. ${ }^{304}$

Spin-splitting effects on an arsenene $/ \mathrm{FeCl}_{2}$ heterostructure were investigated by Song et al., ${ }^{305}$ who showed that due to the interfacial coupling between As $p, \mathrm{Cl} p$, and $\mathrm{Fe} d$ states, spin splitting of $123 \mathrm{meV}$ appears at the conduction band minimum of arsenene. However, this can be tuned by a perpendicular electric field, which can give rise to a 
conversion from an Ohmic contact into a Schottky barrier for specific directions. Bulk metals in contact with arsenene monolayers lead to metallization and form a Schottky barrier in the range of $0.12-0.50 \mathrm{eV}$ for electrons in $\mathrm{Sc}, \mathrm{Ti}, \mathrm{Ag}, \mathrm{Cu}$, and $\mathrm{Au}$ contacts, and barriers of 0.75 and $0.78 \mathrm{eV}$ for holes in Pd and Pt contacts. ${ }^{306}$ Other studies considered the optical properties of arsenene on $\mathrm{MoS}_{2}$, and silicene substrates. $^{307,308} h b$-As/graphene heterostructures have shown a transition from the p-type Schottky barrier $(0.18 \mathrm{eV})$ to the n-type Schottky barrier $(0.31 \mathrm{eV})$ by increasing the interlayer distance from 2.8 to $4.5 \AA^{309}$ Therefore, it was predicted that the Schottky barrier and contact can be tuned effectively by using the interlayer distance or by an applying external electric field. ${ }^{309,310}$ It is obtained that for each interlayer distance case, a linear Dirac-like dispersion around the Fermi level can be well preserved. ${ }^{309}$ In contrast to $h b$-As/graphene, $h b$-As/silicene heterostructures could attain an opened direct bandgap of about $125 \mathrm{meV}$ at the $\mathbf{K}$ high-symmetry point of silicene. This bandgap is linearly controllable over a wide range. The increased electric field gives rise to blue-shift in the optical absorption due to the increasing bandgap. ${ }^{311} h b$-As/GaS heterostructure has been found to be an indirect-band-gap semiconductor with intrinsic type-II band alignment and this vdW heterostructure is a potential photocatalyst for water splitting. ${ }^{312} h b-\mathrm{As} / \mathrm{Ca}(\mathrm{OH})_{2}$ heterostructures have been found to be semiconductors with indirect bandgaps. Tensile strains can be applied to tune the band edge positions and optical absorption for a better match with the redox potentials of water and the solar spectrum, such that the Arsenene/ $\mathrm{Ca}(\mathrm{OH})_{2}$ heterostructure could also become a promising photocatalyst for water splitting. ${ }^{313}$

The effect of germanene and $\mathrm{Ge}(111)$ substrates on the electronic properties of antimonene monolayer was investigated within DFT. ${ }^{46}$ Antimonene overlayers on metal or semiconductor substrates have been also actively investigated. Zhang et al. ${ }^{314}$ pointed out that weak $\mathrm{vdW}$ interactions between a single layer antimonene and graphene, preserves both structures, leaves the intrinsic properties of constituents intact, but a Schottky diode forms upon vertically applied electric field. Furthermore, n-type Schottky barrier to p-type Schottky barrier transition occurred when the interlayer distance was decreased down to $3.1 \AA$. Antimonene partially retained its indirect bandgap of $1.22 \mathrm{eV}$. Once antimonene is on the graphene substrate, ${ }^{315}$ the vertical external electric field can control the Schottky barrier, as well as Schottky and Ohmic contacts at the antimonene/graphene interface. Similar to $h b$ $\mathrm{As} / \mathrm{Si}$, a sizeable bandgap opens at the Dirac point of the $h b-\mathrm{Sb} / \mathrm{Si}$ heterostructure. ${ }^{316}$ In addition, there are some studies, which consider the $h b$-Sb structure as a substrate material. For instance, Liu et al. studied $\mathrm{h}-\mathrm{BN} / h b$-Sb, $h b$-As/ $h b$-Sb, and graphene/ $h b$-Sb heterostructures, and they found that $\mathrm{h}-\mathrm{BN} / h b-\mathrm{Sb}$ and $h b-\mathrm{As} / h b$-Sb are semiconductors with 0.14 and $0.31 \mathrm{eV}$ gaps, respectively, while the graphene/ $h b$-Sb heterostructure is metallic. ${ }^{317}$ In another study on germanene/ $h b$-Sb, it is reported that the calculated binding energy per Ge atom is up to $283 \mathrm{meV}$, which shows that bonding between the germanene and $h b$ $\mathrm{Sb}$ occurs by orbital hybridization or electrostatic interaction in addition to the weak vdW interaction. ${ }^{318}$

Finally, bismuthene-metal contacts have also been recently studied. ${ }^{319}$ It was found that bismuthene forms an n-type Schottky contact with $\mathrm{Ag}$, Ti, and Ir electrodes and a p-type contact with $\mathrm{Al}$, $\mathrm{Au}$, and Pt electrodes; undergoing metallization, with Ohmic contact appearing in the vertical direction. When the $\mathrm{Bi}(111)$ bilayer was placed on the hBN substrate, this was found to support the topological insulating phase of the Bi bilayer, whereas on $\mathrm{SiC}$, large spin and energy splittings were observed, promising for spintronics applications. $^{3}$

\section{COMPOUNDS AND ALLOYS}

Unusual properties have been achieved in compounds or alloys constituted of group-VA elements. ${ }^{322-324}$ For instance, a chemically ordered antimony arsenide (SbAs) compound which has rhombohedral crystal symmetry (A7 structure) is synthesized and found to be a semimetal. The bulk alloy of $\mathrm{Bi}_{1-x} \mathrm{Sb}_{x}$ can be semimetallic or semiconducting depending on the composition $x .{ }^{325}$ Monolayers of group-VA compounds exhibit also different properties from their parent constituent monolayers. Zhu et al. investigated the stability of both $s w$ - and $h b$ - $\mathrm{As}_{1-x} \mathrm{P}_{x}$ compounds and showed that they can undergo a structural transition accompanied by direct-indirect bandgap change depending on the compositions $x^{326}$ The SbAs monolayer has an indirect bandgap, while its bilayer displays semimetallic properties. ${ }^{98}$ Liu et al. ${ }^{97}$ investigated the $h b$ structure of AsN, AsP, AsSb, and AsBi compounds, all being semiconductor with a bandgap in the range of $1.06 \mathrm{eV}-2.05 \mathrm{eV}$. While $h b$-AsN is dynamically unstable, $a w-\mathrm{AsN}$ is predicted to be a dynamically stable semiconductor with an indirect bandgap in the visible light range. ${ }^{327-329}$ A different kind of compound formation as a composite or lateral heterostructure of $h b$-As and $h b$ $\mathrm{Sb}$ has been also treated. ${ }^{330}$ Depending on the composition, BiSb heterojunctions can transform from normal band insulators (NIs) to topological insulators (TIs). First-principles calculations predict that the transition point between TI and NI phases is at $x=5$ in $h b$ $\mathrm{Bi}_{x} \mathrm{Sb}_{8-x}$ and at $x=3$ in $a w-\mathrm{Bi}_{x} \mathrm{Sb}_{4-x}$. ${ }^{331}$ The energy gap involved is smaller than those of $h b$-SbBi. ${ }^{332}$ Calculated values of the electronic mobility of $s w$-AsP compound are found to be higher than those of other $2 \mathrm{D}$ monolayers, such as $\mathrm{MoS}_{2}$, silicon or monolayer of $s w$ $\mathrm{P}^{35,333-335}$ Point defects in $h b$-AsP or $a w$-AsP can tune the electronic and magnetic properties of the structures. ${ }^{336}$ It is shown that the phosphorus nitride $(\mathrm{PN})$ alloy also shows excellent anisotropic carrier mobility along specific directions. ${ }^{337}$

\section{EXPERIMENTAL RESULTS}

It is noteworthy that studies of nitrogen monolayers on various substrates go back to the 1970s. Kjerns et al. ${ }^{338}$ investigated nitrogen films adsorbed on Grafoil (a basal-plane-oriented graphite) and showed that ordered $\mathrm{N}_{2}$ molecules can be adsorbed on graphite at low temperatures ranging from 10 to $40 \mathrm{~K}$, but they become disordered at higher temperatures. Later, Trabelsi and coworkers obtained nitrogen monolayer on $\mathrm{MgO}(100)$ for $\mathrm{T}=10-60 \mathrm{~K}$, which consists of highly ordered $\mathrm{N}_{2}$ molecules in the honeycomb structure. ${ }^{339}$ Very recently, two dimensional nitrogen atomic sheets were epitaxially grown on GaAs by using the site-controlled $\mathrm{N} \delta$-doping technique. ${ }^{340}$ They observed a delocalized impurity band at $1.49 \mathrm{eV}$ with increasing Ndoping density. While theoretical studies predict $h b-\mathrm{N}$ as a local minimum, ${ }^{44}$ its synthesis still remains a challenge.

A photoluminescence peak of single layer $\mathrm{P}$ is observed at $1.45 \mathrm{eV} .{ }^{35}$ Also, p-type FETs based on few layer phosphorene were fabricated. Synthesized phosphorene FETs exhibit ambipolar behavior with drain current modulation up to $\sim 10^{5}$, and a field-effect mobility value up to $1.000 \mathrm{~cm}^{2} \mathrm{~V}^{-1} \mathrm{~s}^{-1}$ at room temperature. ${ }^{341}$ Among diverse methods aiming at synthesizing few or single layer phosphorene, ${ }^{342}$ the cleavage with tape, ${ }^{35,341}$ liquid-phase exfoliation, ${ }^{343,344}$ plasma- 

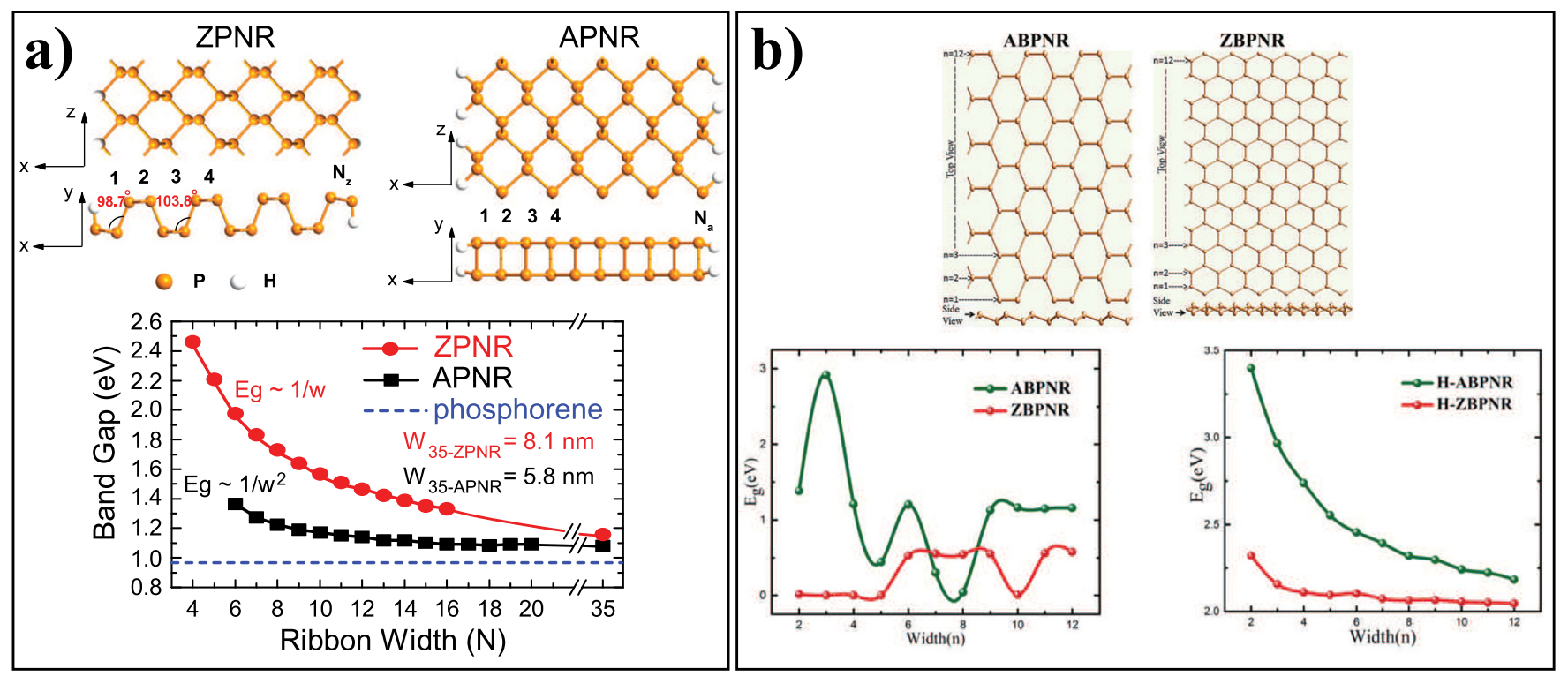

\section{c)}

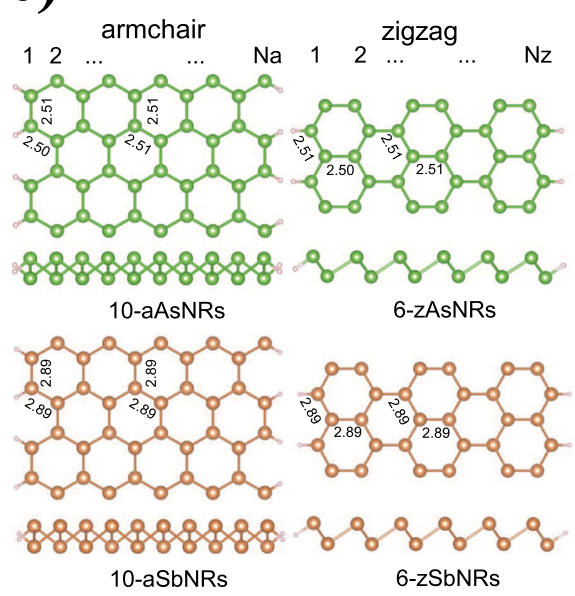

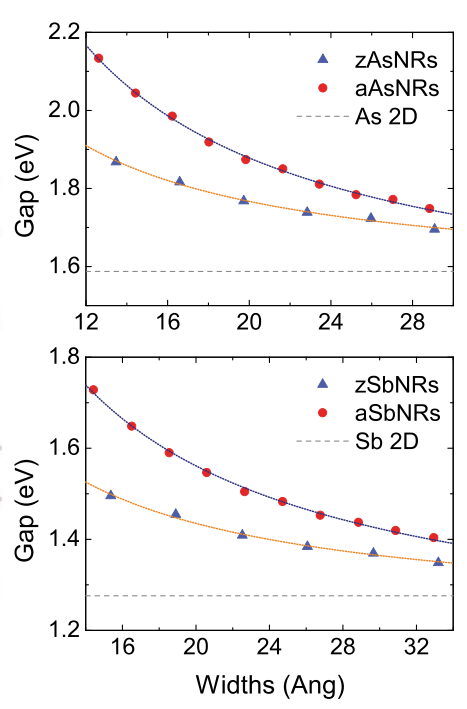

d)

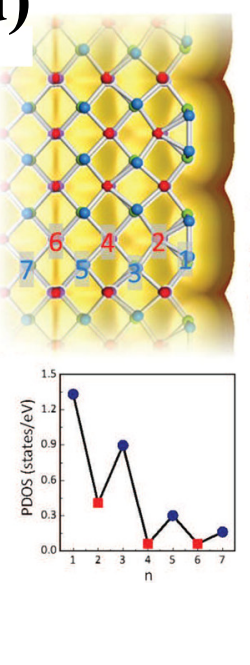

FIG. 9. (a) Top and side views of the optimized geometry structure of hydrogen saturated sw-phosphorene nanoribbons: (Left) zigzag phosphorene nanoribbons (ZPNRs) and (right) armchair phosphorene nanoribbons (APNRs), Bottom panel: variation of the bandgaps of APNRs (up to $5.8 \mathrm{~nm}$ ) and ZPNRs (up to $8.1 \mathrm{~nm}$ ) as a function of ribbon width N. The variation of bandgap is fitted to $\sim 1 / \mathrm{w}$ for ZPNRs and to $\sim 1 / w^{2}$ for APNRs, where $w$ is the width of the ribbon in units of $\AA$. The blue dashed line indicates the bandgap of monolayer phosphorene. Reproduced with permission from Wu et al. Phys. Rev. B 92, 035436 (2015). Copyright 2015 American Chemical Society. (b) Top and side views of ABPNR and ZBPNR blue phosphorene nanoribbons with " $n$ " representing the number of atomic lines in the nanoribbons (top panel). Bandgap, $E_{g}$ versus width, $n$. Reproduced with permission from Swaroop et al. RSC Adv. 7, 2992 (2017). Copyright 2017 Royal Society of Chemistry. (c) Left: The top and lateral views of armchair and zigzag As and Sb nanoribbons. $\mathrm{N}_{a}\left(\mathrm{~N}_{\mathrm{z}}\right.$ ) corresponds to the number of the dimer lines (zigzag chains) across the armchair (zigzag) nanoribbon. The bond lengths at the centre/edges of nanoribbons are indicated. Right: The variation of bandgaps as a function of ribbon widths of As and Sb nanoribbons. The dotted lines correspond to the fittings of data. Reproduced with permission from Wang and Ding Nanoscale Res. Lett. 10, 254 (2015). Copyright 2015 Springer. (d) Simulated STM images of a reconstructed $2 \times 12$ ML Bi(110) film. Projected density of states (PDOS) of halftruncated $2 \mathrm{ML} \mathrm{Bi}(110)$ films as a function of energy. The shaded areas are a guide to the eye, which correspond to characteristic peaks in experiments. The curves labeled in right panel are shifted for clarity. Reproduced with permission from Sun et al., Phys. Rev. Lett. 109, 246804 (2012). Copyright 2012 American Physical Society.

assisted fabrication, ${ }^{341}$ and the Langmuir-Blodgett Assembly method $^{345}$ were used. Gomez et al. ${ }^{346}$ obtained phosphorene by the mechanical exfoliation method, and they found that black phosphorus flakes are very hydrophilic, i.e., they can be etched away upon long term exposure to air moisture. Recent research efforts focused on $2 \mathrm{D}$ nanomaterials for biosensor applications. ${ }^{347,348}$ Cui et al. ${ }^{349}$ fabricated a few-layer phosphorene field effect transistor (FET) by the layer-bylayer deposition method detecting Poly-L-lysine (PLL) linker molecules 
and Alpha-fetoprotein (AFP) antibody. Also, the $s w$-P and poly(3,4ethylenedioxythiophene)-poly(styrenesulfonate) (PEDOT:PSS) composite was prepared by $\mathrm{Li}$ et al. to immobilize hemoglobin $(\mathrm{Hb})$ directly on the electrode. It is suggested that $s w$-P accelerates the direct electrochemistry of $\mathrm{Hb}^{350}$ These results indicate that $s w$-P can be a promising material for biosensing applications. ${ }^{349}$

Despite numerous theoretical predictions about the orthorhombic arsenene (o-As or $s w$-As) monolayers, its successful synthesis has not been achieved yet. According to the calculations by Schmidt et $a .^{351}$ pristine $o$-As is metastable, whereas $\mathrm{As}_{x} \mathrm{P}_{1-x}$ structures are thermodynamically stable. ${ }^{351}$ Zhou et al. ${ }^{352}$ have successfully synthesized the layered $A s_{x} \mathrm{P}_{1-x}$ materials (for $x=0,0.25,0.4$, and 0.83 ) by the exfoliation method. Their results show that the change of $\mathrm{As}_{x} \mathrm{P}_{1-x}$ bandgaps is not linearly dependent on the composition $x$. A year later, Tsai et al. synthesized multilayer arsenene nanoribbons on InAs using the plasma-assisted process. ${ }^{66}$ Nevertheless, this structure is not continuous on the substrate, rather it is like a pile of multilayer nanoribbons. They deduced the structure as washboard, and estimated its bandgap as $2.3 \mathrm{eV} .{ }^{66}$ Very recently, similar to the synthesis of silicene, freestanding like monolayer $h b$-As on $\mathrm{Ag}(111)$ is synthesized by Shah et al. ${ }^{353}$ First, the $\mathrm{Ag}(111)$ substrate was kept at $250-350^{\circ} \mathrm{C}$, and then, it is exposed to As atoms. The experimental value of the indirect bandgap of hb-As was deduced to be $\sim 1.4 \mathrm{eV}^{353}$

Ultrathin antimonene films were grown for the first time on $\mathrm{Bi}_{2} \mathrm{Te}_{3}$ and $\mathrm{Sb}_{2} \mathrm{Te}_{3}$ substrates using the molecular beam epitaxy (MBE) method by Lei et al. ${ }^{354}$ in 2016 . However, it was revealed that the electronic band structure depends on which substrate antimonene was grown on. The same year, Ji et al. ${ }^{355}$ synthesized antimonene via van der Waals epitaxy with very high stability after aging in air for 30 days. ${ }^{355}$ Moreover, it shows high electrical conductivity up to $10^{4}$ $\mathrm{Sm}^{-1}$ and good optical transparency in the visible light range. ${ }^{355}$ Also, like phosphorene, antimonene was synthesized by the liquid-phase exfoliation method ${ }^{288,356}$ and micromechanical exfoliation ${ }^{357}$ Owing to its high stability and antioxidant capacity, antimonene can be a suitable material for optical devices. The experimental results of Song et al. ${ }^{356}$ verify that antimonene based microfibers could operate as a stable nonlinear photonics device. A giant nonlinear refractive index of $\sim 10^{-5} \mathrm{~cm}^{2} \mathrm{~W}^{-1}$ was recently revealed by Lu et al. ${ }^{358}$ These findings herald the utility of antimonene-based photonic devices in communication technologies. Additionally, unlike phosphorene, antimonene does not react with water and is not significantly contaminated upon exposure to ambient conditions. ${ }^{67}$ Following the predictions of Uzengi-Akturk et al., ${ }^{46}$ Deschênes et al. ${ }^{359}$ synthesized antimonene on germanium substrates using solid-source molecular beam epitaxy. Tsai et al. ${ }^{265}$ also fabricated antimonene nanoribbons by the plasmaassisted process on the InSb substrate with a bandgap of $\sim 2.03 \mathrm{eV}$ estimated by photoluminescence measurements at room temperature. Very recently, antimonene in the washboard structure was experimentally synthesized, and found to be topologically nontrivial in the quantum spin Hall class. ${ }^{360}$ Similar to silicene, flat antimonene was grown epitaxially on the $\mathrm{Ag}(111)$ substrate.$^{68}$ Finally, theoretically predicted fluorinated antimonene was synthesized using the method of ionic liquid-assisted electrochemical exfoliation and synchronous fluorination by Zhang et al., ${ }^{361}$ which has a direct bandgap unlike bare antimonene having an indirect bandgap.

An ultrathin Bi film with a puckered structure can be grown on the $\operatorname{Si}(111)-(7 \times 7)$ substrate. However, beyond four atomic layers, it starts to transform into a bulk (001) single crystal at room temperature. ${ }^{184}$ Four monolayer $\mathrm{Bi}(110)$ nanoribbons were epitaxially obtained by Sun et al. ${ }^{69}$ on graphene on $4 \mathrm{H}-\mathrm{SiC}(0001)$ with a bandgap of $0.4 \mathrm{eV}$ measured at the center of the ribbon. Lu et al. revealed that 2 and 4 monolayers of $\mathrm{Bi}(110)$ films with a flat surface are good elemental 2D materials with nontrivial topological properties. ${ }^{362}$ Their experimental results and first principles calculations showed that the topological nature of $\mathrm{Bi}(110)$ films is sensitive to the atomic buckling which is tunable via charge doping and could be controlled by choosing different substrates on which $\mathrm{Bi}(110)$ films are grown. ${ }^{362}$ While most $\mathrm{Bi}$ ultrathin films were grown on the $\mathrm{Si}(111)$ substrate, ${ }^{363-366}$ recently, Reis et al. ${ }^{367}$ synthesized a Bi honeycomb structure with a relatively large gap of $0.8 \mathrm{eV}$ on top of $\mathrm{SiC}(0001)$. Few-layers bismuthene, which was synthesized very recently by the sonochemical exfoliation method had an optical pulse value of 652 femtoseconds and is now considered as an excellent material for ultrafast absorber devices. ${ }^{368}$ Bismuth nanoribbons, which have been produced by the potentiostatic electrochemical method display high electrocatalytic abilities for the detection of biological poisoning of heavy metal ions, such as $\mathrm{Pb}$ and $\mathrm{Cd}$, hence can be utilized as biosensors. ${ }^{369}$

\section{CONCLUSIONS AND OUTLOOKS}

This review article presents an overview of $2 \mathrm{D}$ monolayers and multilayers of group-VA elements, providing a concise discussion on their diverse allotropes together with the atomic structure, stability, and mechanical properties, unusual electronic, optical, magnetic, and thermal properties unveiled so far, recent progress made in their synthesis, as well as on experimental findings heralding potential technological applications. Based on the extensive theoretical and experimental studies, a majority of $2 \mathrm{D}$ structures discussed in this review range from semimetal to wide bandgap semiconductor and hence comprise a wide range of electronic and optical properties. In addition, $2 \mathrm{D}$ structures of $\mathrm{Sb}$ and $\mathrm{Bi}$, and their compounds with a strong spin-orbit coupling, narrow bandgap, and band inversions are candidates for topologically nontrivial behavior and diverse quantum states. In view of the discussions in this review, one concludes that $2 \mathrm{D}$ structures of group-VA elements constitute a class of materials comprising extensive theoretical and experimental research potential, insofar hiding fundamental breakthroughs. Of course, the realization of all theoretically predicted results requires the fabrication/synthesis of the structures under study. We believe that the growth of new materials and their functionalization offer new challenges. In particular, the growth of hexagonal buckled nitrogene is fundamentally critical. Mechanical exfoliation from parent, layered 3D crystals like graphite has been a prevailing procedure to obtain few-layers or monolayer. Even if this method worked for graphene ${ }^{4,5}$ very well, it is not suitable for its "cousins" such as silicene or germanene. On the other hand, recent experimental attempts to obtain few-layers or monolayer of group-VA structures through exfoliation showed that only $s w$-phospherene can be obtained by mechanical exfoliation method, while other group-VA structures can be synthesized only on substrates by epitaxial growth techniques.

In view of the previous research studies, there is an open field for crucial features which need to be further investigated. For example, while the electronic and optical properties depend on the stacking sequence and can be tuned by the number of layers, the nature of interlayer interaction in multilayers is not thoroughly understood. A 
a) AA-stacking
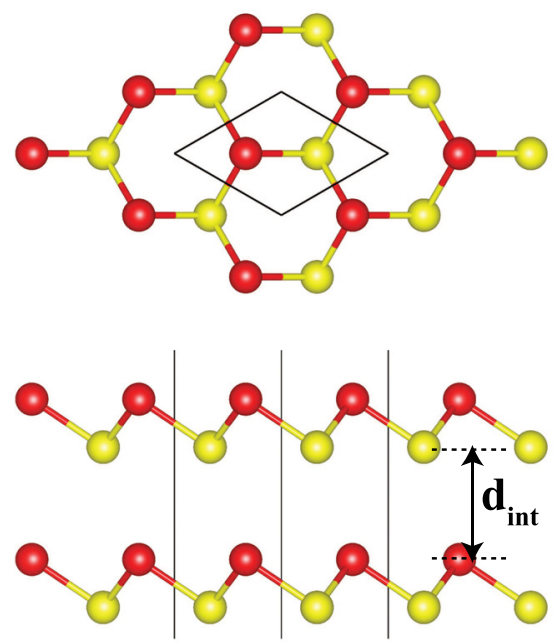

b)

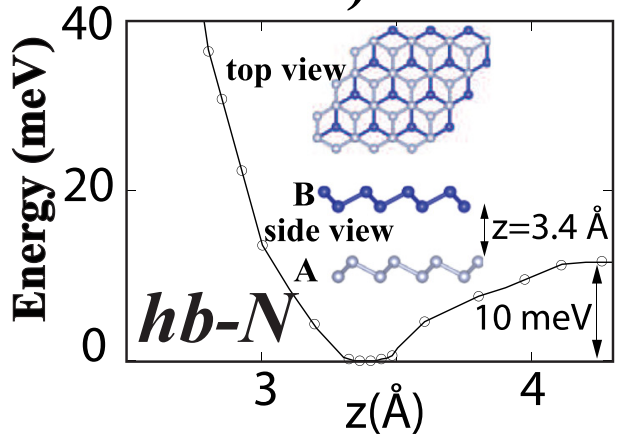

d) $h b$-As (AA-stacking)

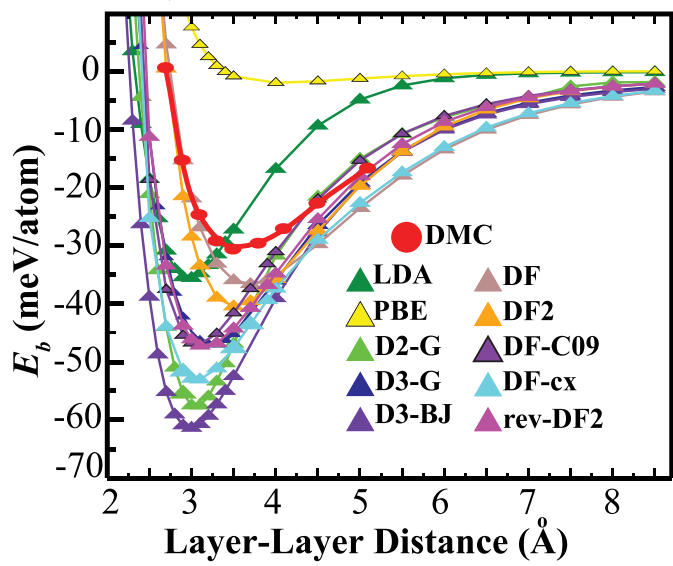

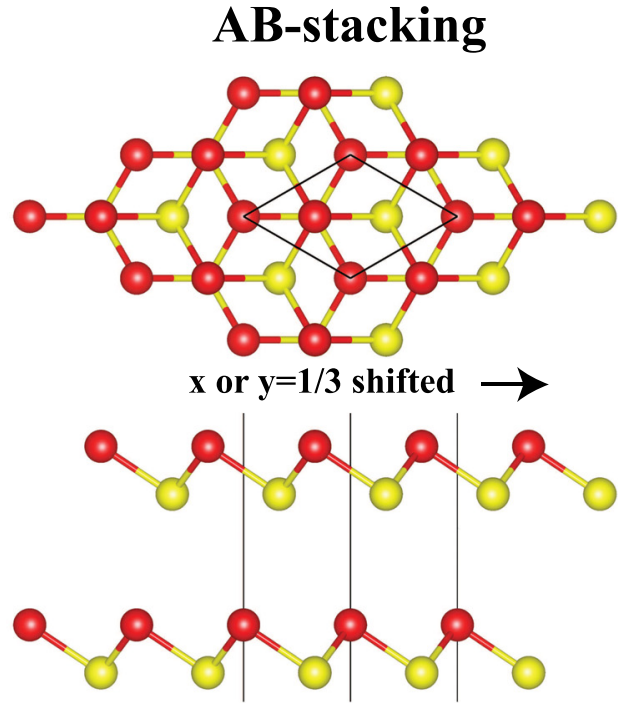

c)

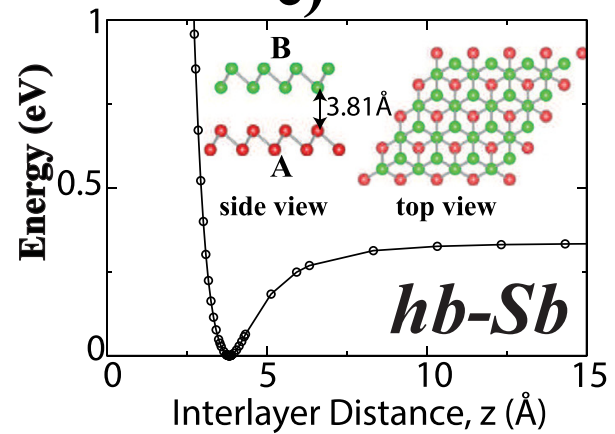

$h b$-As (AB-stacking)

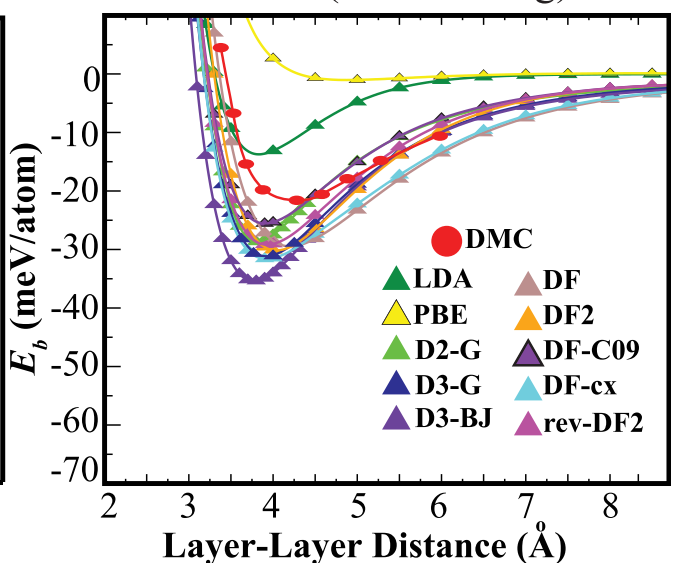

FIG. 10. (a) AA stacking and AB stacking of bilayers formed by monolayers in the $h b$-structure. Top and side views. Reproduced with permission from Kadioglu et al., J. Chem. Phys. 148, 214706 (2018). Copyright 2018 AIP Publishing. (b) Variation of interlayer interaction energy with separation $z$ in the bilayer of $h b$-nitrogene having AB stacking. Reproduced with permission from Özçelik et al. Phys. Rev. B 92, 125420 (2015). Copyright 2015 American Physical Society. (c) Same for the bilayer of $h b$-antimonene. Reproduced with permission from Aktürk et al. Phys. Rev. B 91, 235446 (2015). Copyright 2015 American Physical Society. (d) DFT and DMC (diffusion Monte Carlo) calculation results of binding energies per atom as a function of interlayer spacing for AA- and AB-stacking of bilayer hb-arsenene. Reproduced with permission from Kadioglu et al., J. Chem. Phys. 148, 214706 (2018). Copyright 2018 AIP Publishing. 
a) AA-stacking
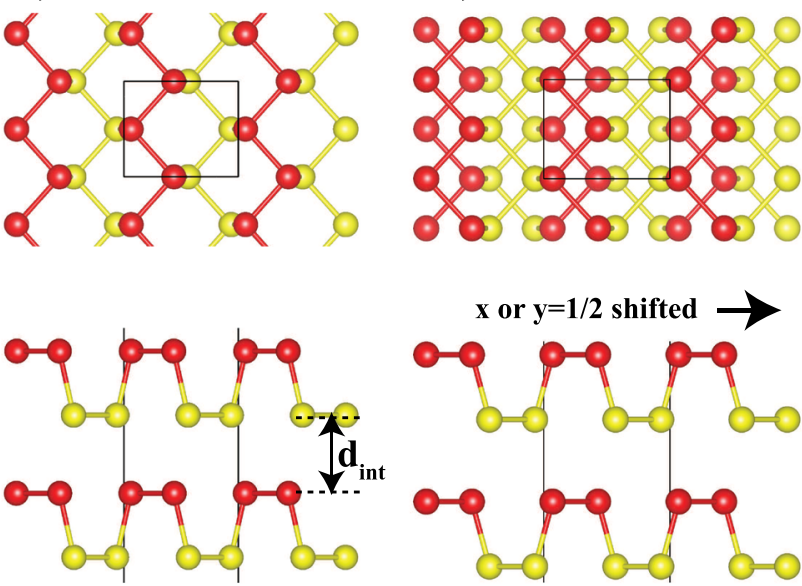

b) AB-stacking

e)
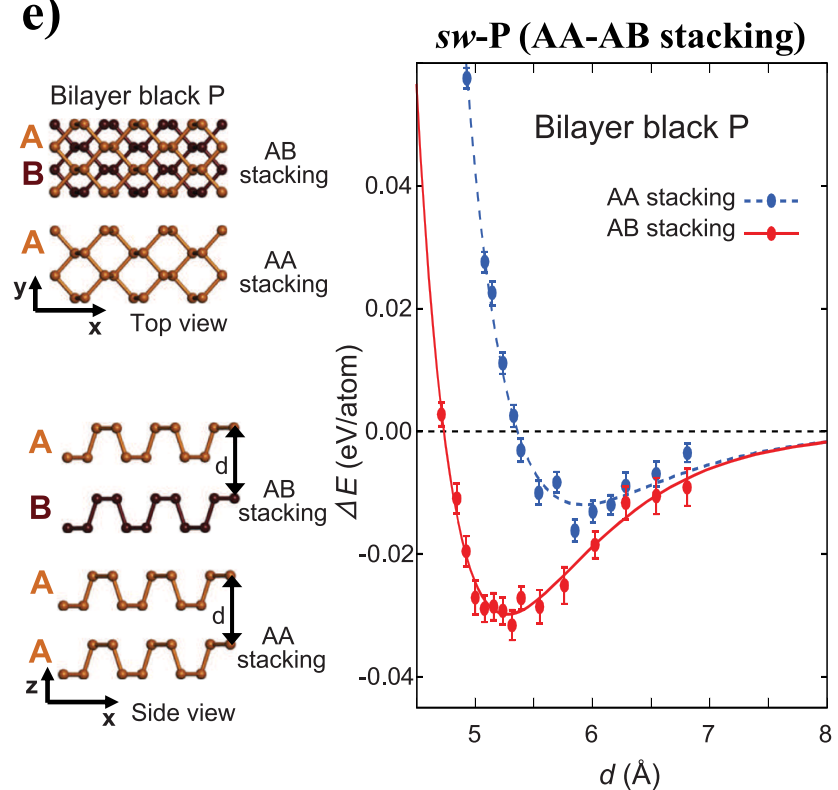

c) AC-stacking
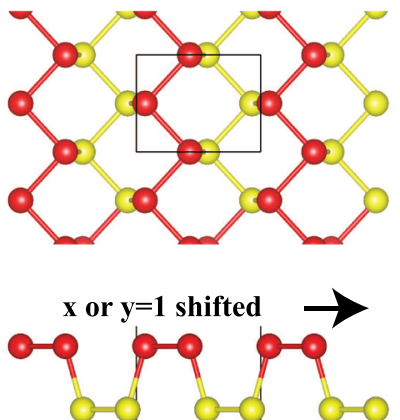

Reflection Plane

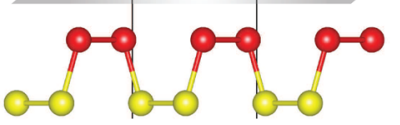

f) $s w$-As (AA-stacking)

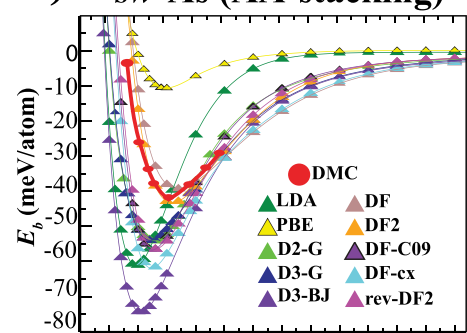

$s w$-As (AC-stacking)

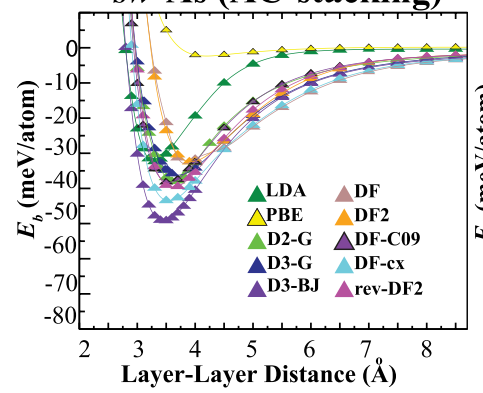

d) AD-stacking
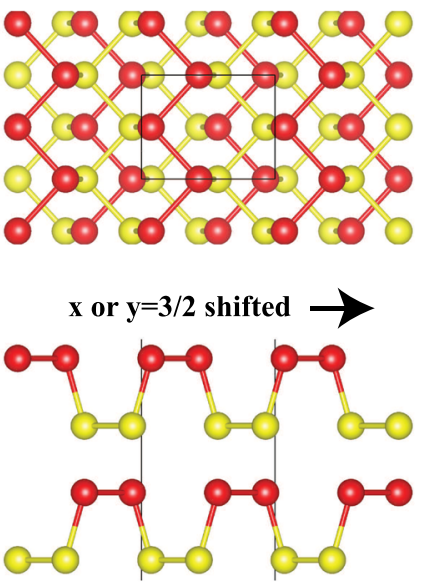
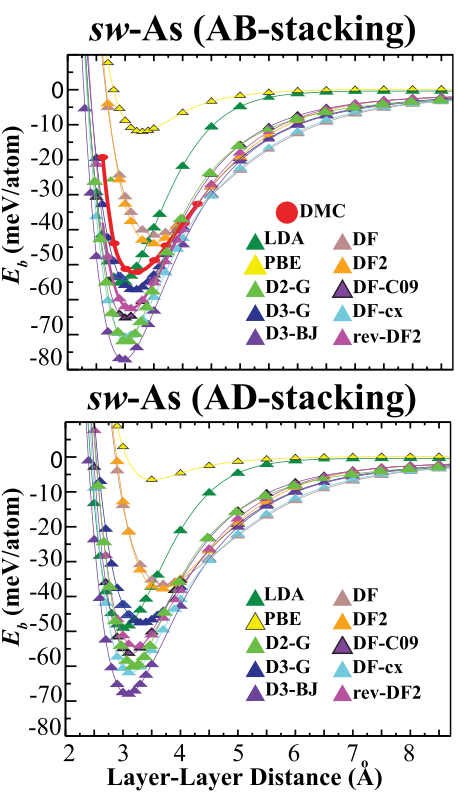

FIG. 11. Top and side views of atomic configurations of bilayers in washboard structures. (a) AA stacking, (b) AB stacking, (c) AC stacking, (d) AD stacking, (e) and (f) variations of interlayer interaction energies with layer spacing for bilayers formed by the monolayers in the washboard structure having different stacking sequences. Reproduced with permissions from Kadioglu et al., J. Chem. Phys. 148, 214706 (2018). Copyright 2018 AIP Publishing; Shulenburger et al., Nano Lett. 15, $8170-8175$ (2015). Copyright 2018 American Chemical Society.

few theoretical studies suggest that multilayers of some group-VA elements differ from van der Waals solids due to the significant chemical interaction between the layers. In this respect, the strength of chemical interaction between the layers needs to be investigated extensively to reveal whether vertical bonds can form. Another topic that needs to be considered for future research is the lateral and vertical heterostructures of the monolayers of group-VA elements. While group-VA/graphene, group-VA/metal heterostructures are intensively examined, single and multiple heterostructures of constituted from the junctions of different group-VA monolayers or multilayers forming the semiconductor/semiconductor junction or quantum structures can be a fruitful subject of research offering interesting applications. In particular, the heterostructures formed by the junction of topologically nontrivial/trivial monolayers is of current interest. Although many theoretical studies prove the energetically or dynamically stable vertical group-VA/2D-substrate heterostructures, there is no study heralding the synthesis of a heterostructure with a monolayer of the group-VA structure, yet. However, the few layer $h b-\mathrm{P} / \mathrm{h}-\mathrm{BN}$ heterostructure is fabricated and remained stable for more than $300 \mathrm{~h}$ in ambient conditions. This result can pave the way to the synthesis of a stable 

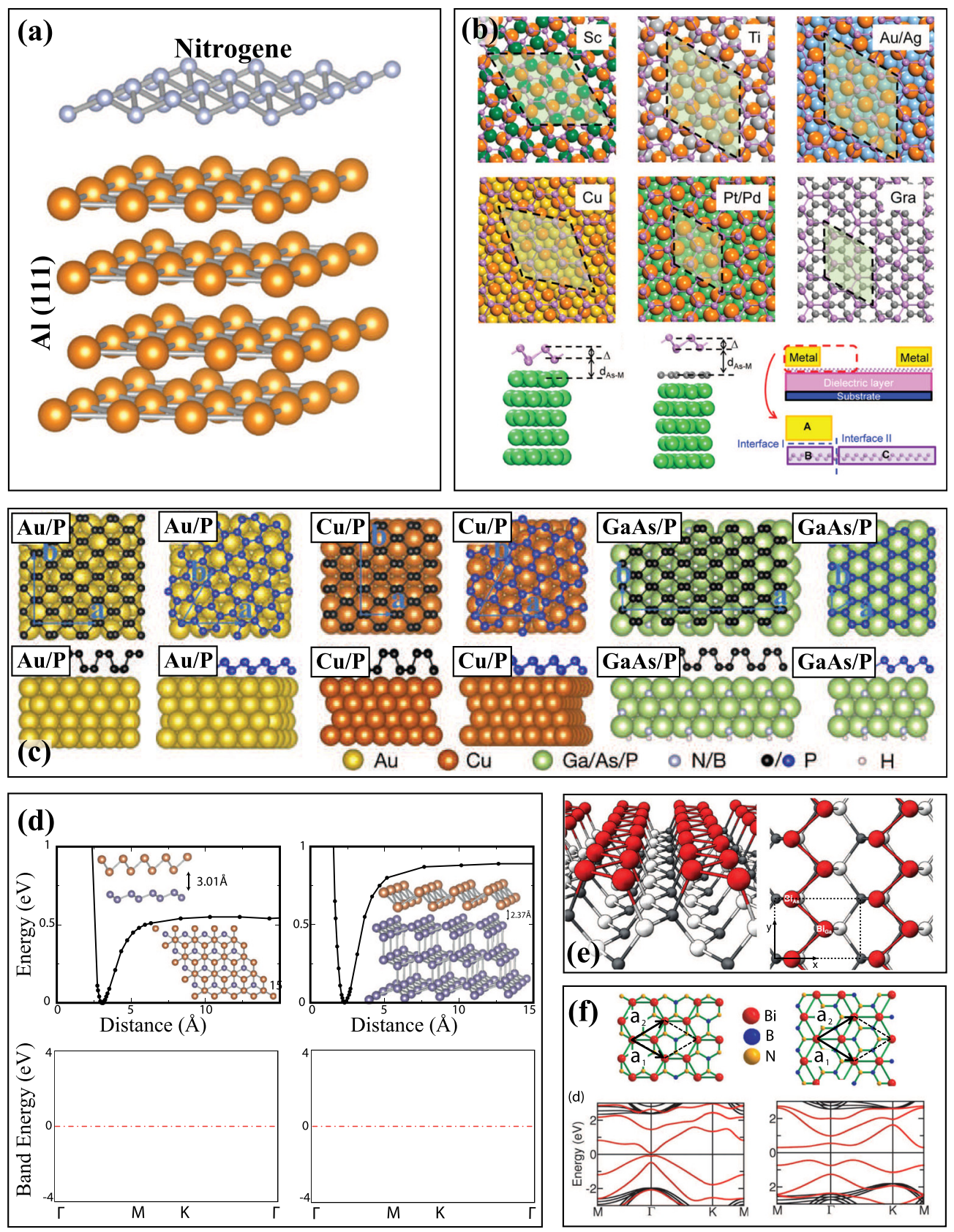

FIG. 12. (a) Atomic configuration of nitrogene overlayer on the Al(111) substrate (or slab) consisting of four Al(111) planes. Small (light blue) and large (yellow) balls denote $\mathrm{N}$ and Al atoms, respectively. Reproduced with permissions from Ozçelik et al., Phys. Rev. B 92, 125420 (2015). Copyright 2015 American Physical Society. (b) Optimized interfacial structures of $\mathrm{ML}$ arsenene contacted to various metal surfaces. Top views of $\mathrm{ML}$ arsenene on the $\mathrm{Sc}(0001), \mathrm{Ti}(0001), \mathrm{Au} / \mathrm{Ag}(111), \mathrm{Cu}(111)$, Pt/Pd(111), and graphene surfaces. The rhombus with a black dashed edge shows the unit cell for each structure. Side views of ML arsenene-Pt contact and ML arsenene-graphene-Pt contact. A schematic view of an ML arsenene FET. Reproduced with permissions from Wang et al. ACS Appl. Mater. Interfaces 9, 29273-29284 (2017). Copyright 2017 American Chemical Society. (c) Top views of the overlayers (either black phosphorene or blue phosphorene) on the substrates [Au(111), Cu(111), GaAs(001)]. Reproduced with permissions from Zeng et al. Phys. Rev. Lett. 118, 046101 (2017). Copyright 2017 American Physical Society. (d) Binding interaction of $h b$-Sb grown on the germanene surface and the corresponding electronic energy band structure. The registry of $\mathrm{Sb}$ and $\mathrm{Ge}$ atoms is shown in the inset. Same for $h b$-Sb grown on the $\mathrm{Ge}(111)$ substrate. The zero of energy is set to the Fermi level shown by dash-dotted line. Reproduced with permissions from Aktürk et al., Phys. Rev. B 91, 235446 (2015). Copyright 2015 American Physical Society. (e) Perspective and top views of the Bi zigzag chain placed on the GaAs(110) surface. ${ }^{321}$ Reproduced with permissions from Kim and Cho Phys. Rev. B 92, 085303 (2015). Copyright 2015 American Physical Society. (f) Left: Bi bilayer on $h-B N \sqrt{3} \times \sqrt{3}$ and Right: Bi bilayer on the $h-B N 2 \times 2$ substrate and corresponding energy bands with red bands being derived from Bi and black bands from the substrate. Reproduced with permissions from Huang et al., Phys. Rev. B. 88, 165301 (2013). Copyright 2013 American Physical Society. 
monolayer of group-VA/2D-substrate heterostructures or other stable few layer group-VA/substrate heterostructures.

Some of the 2D monolayers of group-VA elements display strong directionality in diverse physical properties. This behavior can be implemented in the in-plane, commensurate lateral heterostructures, to produce $2 \mathrm{D}$ monolayers consisting of parallel stripes of group-VA elements. Such composite mono or multilayers can offer interesting technological applications. Similar effects can be realized by the monatomic chains of group-VA elements attached to their monolayers. In this respect, the research on monatomic chains by further lowering the dimensionality from $2 \mathrm{D}$ to strictly $1 \mathrm{D}$ is expected to unveil interesting and fundamental properties.

Considering biological activities based on phosphorene revealed in many previous studies, the decoration of group-VA monolayers by organic molecules, in particular, self-assembly of organic molecules such as amino acids on phosphorene, as well as on others can be potential research directions, both theoretically and experimentally.

Briefly, this review article has shown that 2D monolayers of group-VA elements display important mechanical, optoelectronic, magnetic, and thermal properties, which can be further modified by forming multilayers, junctions, adatom, and organic molecule coating, patterned defects, constructing compounds or composites. These structures attaining novel properties have the potential to generate further research activities. We hope and expect that this review will be a platform summarizing this rapidly developing subject for the reader who can make further fundamental contributions and seminal discoveries mediating useful applications.

\section{ACKNOWLEDGMENTS}

This work was supported by the Scientific and Technological Research Council of Turkey (TUBITAK) under Project No. 117F241. E.D. acknowledges support from The Turkish Academy of Sciences Outstanding Young Scientists Award Program (TUBA-GEBIP). S.C. acknowledges the financial support from the Academy of Sciences of Turkey (TUBA).

\section{REFERENCES}

${ }^{1}$ A. K. Geim and I. V. Grigorieva, Nature 499, 419 (2013).

${ }^{2}$ S. Z. Butler, S. M. Hollen, L. Cao, Y. Cui, J. A. Gupta, H. R. Gutierrez, T. F. Heinz, S. S. Hong, J. Huang, A. F. Ismach et al., ACS Nano 7(4), 2898 (2013).

${ }^{3}$ Q. H. Wang, K. Kalantar-Zadeh, A. Kis, J. N. Coleman, and M. S. Strano, Nat. Nanotechnol. 7, 699 (2012).

${ }^{4}$ K. S. Novoselov, D. Jiang, F. Schedin, T. J. Booth, V. V. Khotkevich, S. V. Morozov, and A. K. Geim, PNAS 102, 10451 (2005).

${ }^{5}$ K. S. Novoselov, A. K. Geim, S. V. Morozov, D. Jiang, M. I. Katsnelson, I. V. Grigorieva, S. V. Dubonos, and A. A. Firsov, Nature 438, 197 (2005).

${ }^{6}$ D. Pacile, J. C. Meyer, C. O. Girit, and A. Zettl, Appl. Phys. Lett. 92, 133107 (2008).

${ }^{7}$ M. Topsakal, E. Aktürk, and S. Ciraci, Phys. Rev. B 79, 115442 (2009).

${ }^{8}$ E. Durgun, S. Tongay, and S. Ciraci, Phys. Rev. B 72, 075420 (2005).

${ }^{9}$ S. Cahangirov, M. Topsakal, E. Aktürk, H. Sahin, and S. Ciraci, Phys. Rev. Lett. 102, 236804 (2009).

${ }^{10}$ H. Sahin, S. Cahangirov, M. Topsakal, E. Bekaroglu, E. Akturk, R. T. Senger, and S. Ciraci, Phys. Rev. B 80, 155453 (2009).

${ }^{11}$ P. Vogt, P. DePadova, C. Quaresima, J. Avila, E. Frantzeskakis, M. C. Asensio, A. Resta, B. Ealet, and G. LeLay, Phys. Rev. Lett. 108, 155501 (2012).

${ }^{12}$ V. O. Özçelik, E. Durgun, and S. Ciraci, J. Phys. Chem. Lett. 5, 2694 (2014).

${ }^{13}$ D. Kecik, A. Onen, M. Konuk, E. Gürbüz, F. Ersan, S. Cahangirov, E. Aktürk, E. Durgun, and S. Ciraci, Appl. Phys. Rev. 5, 011105 (2018).
${ }^{14}$ Y. Xu, B. Yan, H. J. Zhang, J. Wang, G. Xu, P. Tang, W. Duan, and S. C. Zhang, Phys. Rev. Lett. 111, 136804 (2013).

${ }^{15}$ L. Tao, E. Cinquanta, D. Chiappe, C. Grazianetti, M. Fanciulli, M. Dubey, A. Molle, and D. Akinwande, Nat. Nanotechnol. 10, 227 (2015).

${ }^{16}$ V. O. Özçelik, S. Cahangirov, and S. Ciraci, Phys. Rev. Lett. 112, 246803 (2014).

${ }^{17}$ C. Ataca, H. Sahin, and S. Ciraci, J. Phys. Chem. C 116, 8983 (2012).

${ }^{18}$ F. Ersan, H. D. Ozaydin, and O. Uzengi Akturk, Philos. Mag. 99, 376 (2019).

${ }^{19}$ P. Joensen, R. F. Frindt, and S. R. Morrison, Mater. Res. Bull. 21, 457 (1986).

${ }^{20}$ J. N. Coleman, M. Lotya, A. O’Neill, S. D. Bergin, P. J. King, U. Khan, and K. Young, Science 331, 568 (2011).

${ }^{21}$ C. Ataca and S. Ciraci, J. Phys. Chem. C 115, 13303 (2011).

${ }^{22}$ F. Ersan, S. Cahangirov, G. Gokoglu, A. Rubio, and E. Akturk, Phys. Rev. B 94, 155415 (2016).

${ }^{23}$ J. R. Brent, D. J. Lewis, T. Lorenz, E. A. Lewis, N. Savjani, S. J. Haigh, G. Seifert, B. Derby, and P. O'Brien, J. Am. Chem. Soc. 137(39), 12689 (2015).

${ }^{24}$ L. D. Zhao, S. H. Lo, Y. Zhang, H. Sun, G. Tan, C. Uher, C. Wolverton, V. P. Dravid, and M. G. Kanatzidis, Nature 508, 373 (2014).

${ }^{25}$ F. O. von Rohr, H. Ji, F. A. Cevallos, T. Gao, N. P. Ong, and R. J. Cava, J. Am. Chem. Soc. 139(7), 2771 (2017).

${ }^{26}$ V. O. Özçelik, K. Gong, and C. E. White, Nano Lett. 18(3), 1786 (2018).

${ }^{27}$ A. Suslu, K. Wu, H. Sahin, B. Chen, S. Yang, H. Cai, T. Aoki, S. Horzum, J. Kang, F. M. Peeters, and S. Tongay, Sci. Rep. 6, 20525 (2016).

${ }^{28}$ L. Rapoport, Y. Bilik, Y. Feldman, M. Homyonfer, S. Cohen, and R. Tenne, Nature 387, 791 (1997).

${ }^{29}$ C. Lee, Q. Li, W. Kalb, X.-Z. Liu, H. Berger, R. W. Carpick, and J. Hone, Science 328, 76 (2010).

${ }^{30}$ S. Cahangirov, C. Ataca, M. Topsakal, H. Sahin, and S. Ciraci, Phys. Rev. Lett. 108, 126103 (2012).

${ }^{31}$ M. Yu. Arsentev, A. V. Petrov, A. B. Missyul, and M. Hammouri, RSC Adv. 8, 26169 (2018).

${ }^{32}$ F. Ersan, H. D. Ozaydin, G. Gökoglu, and E. Akturk, Appl. Surf. Sci. 425, 301 (2017).

${ }^{33}$ L. Li, Y. Yu, G. J. Ye, Q. Ge, X. Ou, H. Wu, D. Feng, X. H. Chen, and Y. Zhang, Nat. Nanotechnol. 9, 372 (2014).

${ }^{34}$ Z. Zhu and D. Tománek, Phys. Rev. Lett. 112, 176802 (2014).

${ }^{35}$ H. Liu, A. T. Neal, Z. Zhu, Z. Luo, X. Xu, D. Tománek, and P. D. Ye, ACS Nano 8, 4033 (2014).

${ }^{36}$ M. Buscema, D. J. Groenendijk, S. I. Blanter, G. A. Steele, H. S. J. van der Zant, and A. Castellanos-Gomez, Nano Lett. 14, 3347 (2014).

${ }^{37} \mathrm{R}$. Fei and L. Yang, Nano Lett. 14, 2884 (2014).

${ }^{38}$ F. Xia, H. Wang, and Y. Jia, Nat. Commun. 5, 4458 (2014).

${ }^{39}$ T. Hong, B. Chamlagain, W. Lin, H. J. Chuang, M. Pan, Z. Zhou, and Y. Q. $\mathrm{Xu}$, Nanoscale 6, 8978 (2014).

${ }^{40}$ A. Chaves, T. Low, P. Avouris, D. Cakir, and F. M. Peeters, Phys. Rev. B 91, 155311 (2015).

${ }^{41}$ R. Xu, J. Yang, Y. Zhu, H. Yan, J. Pei, Y. W. Myint, S. Zhang, and Y. Lu, Nanoscale 8, 129 (2016).

${ }^{42}$ R. Xu, J. Yang, Y. W. Myint, J. Pei, H. Yan, F. Wang, and Y. Lu, Adv. Mater. 28, 3493 (2016).

${ }^{43}$ Vy. Tran, R. Soklaski, Y. Liang, and L. Yang, Phys. Rev. B 89, 235319 (2014).

${ }^{44}$ V. O. Özçelik, O. Ü. Aktürk, E. Durgun, and S. Ciraci, Phys. Rev. B 92, 125420 (2015).

${ }^{45}$ C. Kamal and M. Ezawa, Phys. Rev. B 91, 085423 (2015).

${ }^{46}$ O. Ü. Aktürk, V. O. Özçelik, and S. Ciraci, Phys. Rev. B 91, 235446 (2015).

${ }^{47}$ Z. Zhang, Z. Yan, Y. Li, Z. Chen, and H. Zeng, Angew. Chem. Int. Ed. 54, 3112 (2015).

${ }^{48}$ E. Aktürk, O. Ü. Aktürk, and S. Ciraci, Phys. Rev. B 94, 014115 (2016).

${ }^{49}$ F. Ersan, E. Aktürk, and S. Ciraci, Phys. Rev. B 94, 245417 (2016).

${ }^{50}$ D. Kecik, E. Durgun, and S. Ciraci, Phys. Rev. B 94, 205409 (2016).

${ }^{51}$ Z. D. Sha, Q. X. Pei, Z. Ding, J. W. Jiang, and Y. W. Zhang, J. Phys. D: Appl. Phys. 48, 395303 (2015).

${ }^{52}$ V. Eswaraiah, Q. Zeng, Y. Long, and Z. Liu, Small 12(26), 3480 (2016).

${ }^{53}$ R. Gusmão, Z. Sofer, and M. Pumera, Angew. Chem. Int. Ed. 56, 8052 (2017).

${ }^{54}$ S. Balendhran, S. Walia, H. Nili, S. Sriram, and M. Bhaskaran, Small 11(6), 640 (2015). 
${ }^{55}$ A. Carvalho, M. Wang, X. Zhu, A. S. Rodin, H. Su, and A. H. C. Neto, Nat. Rev. Mater. 1, 16061 (2016).

${ }^{56}$ Y. Jing, X. Zhang, and Z. Zhou, WIREs Comput. Mol. Sci. 6, 5 (2016).

${ }^{57}$ A. Khandelwal, K. Mani, M. H. Karigerasi, and I. Lahiri, Mater. Sci. Eng. B 221, 17 (2017).

${ }^{58}$ X. Ling, H. Wang, S. Huang, F. Xia, and M. S. Dresselhaus, PNAS 112(15), 4523 (2015).

${ }^{59}$ S. Zhang, S. Guo, Z. Chen, Y. Wang, H. Gao, J. G. Herrero, P. Ares, F. Zamora, Z. Zhu, and H. Zeng, Chem. Soc. Rev. 47(3), 982 (2018).

${ }^{60}$ X. Ren, P. Lian, D. Xie, Y. Yang, Y. Mei, X. Huang, Z. Wang, and X. Yin, J. Mater. Sci. 52, 10364 (2017).

${ }^{61}$ A. J. Mannix, B. Kiraly, M. C. Hersam, and N. P. Guisinger, Nat. Rev. Chem. 1, 0014 (2017).

${ }^{62}$ P. Ares, J. J. Palacios, G. Abellán, J. G. Herrero, and F. Zamora, Adv. Mater. 30(2), 1703771 (2018).

${ }^{63}$ R. Gusmão, Z. Sofer, D. Bouša, and M. Pumera, Angew. Chem. Int. Ed. 56, 14417 (2017).

${ }^{64}$ L. Kou, C. Chen, and S. C. Smith, J. Phys. Chem. Lett. 6, 2794 (2015).

${ }^{65}$ J. L. Zhang, S. Zhao, C. Han, Z. Wang, S. Zhong, S. Sun, R. Guo, X. Zhou, C. D. Gu, K. D. Yuan, Z. Li, and W. Chen, Nano Lett. 16, 4903 (2016).

${ }^{66}$ H. S. Tsai, S. W. Wang, C. H. Hsiao, C. W. Chen, H. Ouyang, Y. L. Chueh, H. C. Kuo, and J. H. Liang, Chem. Mater. 28(2), 425 (2016).

${ }^{67}$ P. Ares, F. A. Galindo, D. R. S. Miguel, D. A. Aldave, S. D. Tendero, M. Alcami, F. Martin, J. G. Herrero, and F. Zamora, Adv. Mater. 28, 6332 (2016).

${ }^{68}$ Y. Shao, Z. L. Liu, C. Cheng, X. Wu, H. Liu, C. Liu, J. O. Wang, S. Y. Zhu, Y. Q. Wang, D. X. Shi, K. Ibrahim, J. T. Sun, Y. L. Wang, and H. J. Gao, Nano Lett. 18, 2133 (2018).

${ }^{69}$ J. T. Sun, H. Huang, S. L. Wong, H. J. Gao, Y. P. Feng, and A. T. S. Wee, Phys. Rev. Lett. 109, 246804 (2012).

${ }^{70}$ S. Zhang, M. Xie, F. Li, Z. Yan, Y. Li, E. Kan, W. Liu, Z. Chen, and H. Zeng, Angew. Chem., Int. Ed. 55, 1666 (2016).

${ }^{71}$ Z. Y. Zhang, H. N. Cao, J. C. Zhang, Y. H. Wang, D. S. Xue, and M. S. Si, AIP Adv. 5, 067117 (2015).

${ }^{72}$ Y. Zhang, J. Lee, W. L. Wang, and D. X. Yao, Comput. Mater. Sci. 110, 109 (2015).

${ }^{73}$ S. Nahas, A. Bajaj, and S. Bhowmick, Phys. Chem. Chem. Phys. 19, 11282 (2017).

${ }^{74}$ X. Kong, L. Li, O. Leenaerts, X. J. Liu, and F. M. Peeters, Phys. Rev. B 96, 035123 (2017).

${ }^{75}$ J. Guan, Z. Zhu, and D. Tománek, ACS Nano 8(12), 12763 (2014).

${ }^{76}$ J. Guan, Z. Zhu, and D. Tománek, Phys. Rev. Lett. 113, 046804 (2014).

${ }^{77}$ Z. Li, C. He, T. Ouyang, C. Zhang, C. Tang, R. A. Romer, and J. Zhong, Phys. Rev. Appl. 9, 044032 (2018).

${ }^{78}$ T. Zhao, C. Y. He, S. Y. Ma, K. W. Zhang, X. Y. Peng, G. F. Xie, and J. X. Zhong, J. Phys.: Condens. Matter 27, 265301 (2015).

${ }^{79}$ T. Zhao, G. Wang, and Y. Liao, Chem. Phys. Lett. 652, 1 (2016).

${ }^{80}$ C. He, C. Zhang, C. Tang, T. Ouyang, J. Li, and J. Zhong, Sci. Rep. 7, 46431 (2017).

${ }^{81}$ H. Wang, X. Li, Z. Liua, and J. Yang, Phys. Chem. Chem. Phys. 19, 2402 (2017).

${ }^{82}$ G. Yu, L. Jiang, and Y. Zheng, J. Phys.: Condens. Matter 27, 255006 (2015).

${ }^{83}$ J. Liu, Y. Guo, S. Zhang, Q. Wang, Y. Kawazoe, and P. Jena, J. Phys. Chem. C 119, 24674 (2015).

${ }^{84}$ J. R. Feng and G. C. Wang, RSC Adv. 6, 22277 (2016).

${ }^{85} \mathrm{M}$. Xu, C. He, C. Zhang, C. Tang, and J. Zhong, Phys. Status Solidi RRL 10(7), 563 (2016).

${ }^{86} \mathrm{H}$. Lv, Z. Zhuo, and X. Wu, "Exploring two-dimensional crystals with atomic thickness from molecular design and global structure search," in Inorganic Two-Dimensional Nanomaterials: Fundamental Understanding, Characterizations and Energy Applications, Smart Materials edited by C. Wu (The Royal Society of Chemistry, 2017), Vol. 27.

${ }^{87}$ S. Mardanya, V. K. Thakur, S. Bhowmick, and A. Agarwal, Phys. Rev. B 94, 035423 (2016).

${ }^{88}$ G. Wang, R. Pandey, and S. P. Karna, ACS Appl. Mater. Interfaces 7, 11490 (2015).

${ }^{89}$ G. Zheng, Y. Jia, S. Gao, and S. H. Ke, Phys. Rev. B 94, 155448 (2016).

${ }^{90}$ J. Lee, W. C. Tian, W. L. Wang, and D. X. Yao, Sci. Rep. 5, 11512 (2015).
${ }^{91}$ M. Sun, S. Wang, J. Yu, and W. Tang, Appl. Surf. Sci. 392, 46 (2017).

${ }^{92}$ W. Yu, Z. Zhu, C. Y. Niu, C. Li, J. H. Cho, and Y. Jia, Nanoscale Res. Lett. 11, 77 (2016).

${ }^{93}$ Y. Mogulkoc, M. Modarresi, A. Mogulkoc, and Y. O. Ciftci, Comput. Mater. Sci. 124, 23 (2016).

${ }^{94}$ V. Vierimaa, A. V. Krasheninnikov, and H. P. Komsa, Nanoscale 8, 7949 (2016).

${ }^{95}$ Q. Wei and X. Peng, Appl. Phys. Lett. 104, 251915 (2014).

${ }^{96}$ J. W. Jiang and H. S. Park, J. Phys. D: Appl. Phys. 47, 385304 (2014).

${ }^{97}$ M. Y. Liu, Y. Huang, Q. Y. Chen, C. Cao, and Y. He, Sci. Rep. 6, 29114 (2016).

${ }^{98}$ L. Kou, Y. Ma, X. Tan, T. Frauenheim, A. Du, and S. Smith, J. Phys. Chem. C 119, 6918 (2015).

99. J. Carrete, L. J. Gallego, and N. Mingo, J. Phys. Chem. Lett. 8, 1375 (2017).

${ }^{100}$ S. Zhang, Z. Yan, Y. Li, Z. Chen, and H. Zeng, Angew. Chem., Int. Ed. 127, 3155 (2015).

${ }^{101}$ Z. Zhang, J. Xie, D. Yang, Y. Wang, M. Si, and D. Xue, Appl. Phys. Express 8, 055201 (2015).

${ }^{102}$ J. Lee, W. C. Tian, W. L. Wang, and D. X. Yao, Sci. Rep. 5, 11512 (2015).

${ }^{103}$ G. Wang, R. Pandey, and S. P. Karna, ACS Appl. Mater. Interfaces 7(21), 11490 (2015).

${ }^{104}$ B. Rasche, G. Seifert, and A. Enyashin, J. Phys. Chem. C 114, 22092 (2010).

${ }^{105}$ L. Kou, X. Tan, Y. Ma, H. Tahini, L. Zhou, Z. Sun, D. Aijun, C. Chen, and S. C. Smith, 2D Mater. 2, 045010 (2015).

${ }^{106}$ J. S. Li, W. L. Wang, and D. X. Yao, Sci. Rep. 6, 34177 (2016).

${ }^{107}$ Y. Aierken, D. Cakir, C. Sevik, and F. M. Peeters, Phys. Rev. B 92, 081408(R) (2015).

${ }^{108}$ J. R. Feng and G. C. Wang, RSC Adv. 6, 22277 (2016).

${ }^{109}$ D. Cakir, H. Sahin, and F. M. Peeters, Phys. Rev. B 90, 205421 (2014).

${ }^{110}$ F. Ersan, E. Aktürk, and S. Ciraci, J. Phys. Chem. C 120, 14345 (2016).

${ }^{11}$ C. Liu, C. S. Liu, and X. Yan, Phys. Lett. A 381, 1092 (2017).

${ }^{112}$ D. Singh, S. K. Gupta, Y. Sonvane, and I. LukaCević, J. Mater. Chem. C 4, 6386 (2016).

${ }^{113}$ R. R. Q. Freitas, R. Rivelino, F. de Brito Mota, C. M. C. de Castilho, A. K. Georgieva, and G. K. Gueorguiev, J. Phys. Chem. C 119, 23599 (2015).

${ }^{114}$ B. Peng, D. Zhang, H. Zhang, H. Shao, G. Ni, Y. Zhu, and H. Zhu, Nanoscale 9, 7397 (2017).

${ }^{115}$ W. Lu, H. Nan, J. Hong, Y. Chen, C. Zhu, Z. Liang, X. Ma, Z. Ni, C. Jin, and Z. Zhang, Nano Res. 7(6), 853 (2014).

${ }^{116}$ J. Zhang, H. J. Liu, L. Cheng, J. Wei, J. H. Liang, D. D. Fan, P. H. Jiang, and J. Shi, Sci. Rep. 7, 4623 (2017).

${ }^{117}$ C. Xiao, F. Wang, S. A. Yang, Y. Lu, Y. Feng, and S. Zhang, Adv. Funct. Mater. 28, 1707383 (2018).

${ }^{118}$ S. Sharma, S. Kumar, and U. Schwingenschlögl, Phys. Rev. Appl. 8, 044013 (2017).

${ }^{119}$ M. Zeraati, S. M. V. Allaei, I. A. Sarsari, M. Pourfath, and D. Donadio, Phys. Rev. B 93, 085424 (2016).

${ }^{120}$ P. Zhang, Z. Liu, W. Duan, F. Liu, and J. Wu, Phys. Rev. B 85, 201410(R) (2012).

${ }^{121}$ K. X. Chen, S. S. Lyu, X. M. Wang, Y. X. Fu, Y. Heng, and D. C. Mo, J. Phys. Chem. C 121, 13035 (2017).

${ }^{122}$ T. Zhang, Y. Y. Qi, X. R. Chen, and L. C. Cai, Phys. Chem. Chem. Phys. 18, 30061 (2016).

${ }^{123}$ S. K. Gupta, Y. Sonvane, G. Wang, and R. Pandey, Chem. Phys. Lett. 641, 169 (2015).

${ }^{124}$ D. Schiferl and C. S. Barrett, J. Appl. Crystallogr. 2, 30 (1969).

${ }^{125}$ M. A. Ortigoza, I. Y. Sklyadneva, R. Heid, E. V. Chulkov, T. S. Rahman, K. P. Bohnen, and P. M. Echenique, Phys. Rev. B 90, 195438 (2014).

${ }^{126}$ X. Gonze, J. P. Michenaud, and J. P. Vigneron, Phys. Rev. B 41, 11827 (1990).

${ }^{127}$ Z. Q. Huang, F. C. Chuang, C. H. Hsu, Y. T. Liu, H. R. Chang, H. Lin, and A. Bansil, Phys. Rev. B 88, 165301 (2013).

${ }^{128}$ L. Cheng, H. Liu, X. Tan, J. Zhang, J. Wei, H. Lv, J. Shi, and X. Tang, J. Phys. Chem. C 118, 904 (2014).

${ }^{129}$ V. Chis, G. Benedek, P. M. Echenique, and E. V. Chulkov, Phys. Rev. B 87, 075412 (2013)

${ }^{130}$ R. W. Zhang, C. W. Zhang, W. X. Ji, S. S. Yand, and Y. G. Yao, Nanoscale 9, 8207 (2017). 
${ }^{131}$ I. Aguilera, C. Friedrich, and S. Blügel, Phys. Rev. B 91, 125129 (2015).

${ }^{132}$ Y. Kadioglu, S. B. Kilic, S. Demirci, O. Ü. Aktürk, E. Aktürk, and S. Ciraci, Phys. Rev. B 96, 245424 (2017).

${ }^{133}$ W. Yu, C.-Y. Niu, Z. Zhu, X. Cai, L. Zhang, S. Bai, R. Zhao, and Y. Jia, RSC Adv. 7, 27816 (2017).

${ }^{134}$ J. P. Perdew, K. Burke, and M. Ernzerhof, Phys. Rev. Lett. 77, 3865 (1996).

${ }^{135}$ J. Heyd, G. E. Scuseria, and M. Ernzerhof, J. Chem. Phys. 118, 8207 (2003); 124, 219906 (2006).

${ }^{136}$ V. O. Özçelik, J. G. Azadani, C. Yang, S. J. Koester, and T. Low, Phys. Rev. B 94, 035125 (2016).

${ }^{137}$ M. Gajdoś, K. Hummer, G. Kresse, J. Furthmüller, and F. Bechstedt, Phys. Rev. B 73, 045112 (2006).

${ }^{138}$ W. Lin, J. Li, W. Wang, S. D. Liang, and D. X. Yao, Sci. Rep. 8, 1674 (2018).

${ }^{139}$ L. Li, Y. Yu, G. J. Ye, Q. Ge, X. Ou, H. Wu, D. Feng, X. Hui Chen, and Y. Zhang, Nat. Nanotechnol. 9, 372 (2014).

${ }^{140}$ R. Fei and L. Yang, Nano Lett. 14, 2884 (2014).

${ }^{141}$ D. Cakir, H. Sahin, and F. M. Peeters, Phys. Rev. B 90, 205421 (2014).

${ }^{142}$ X. Wang, A. M. Jones, K. L. Seyler, V. Tran, Y. Jia, H. Zhao, H. Wang, L. Yang, X. Xu, and F. Xia, Nat. Nanotechnol. 10, 517 (2015).

${ }^{143}$ J. Yang, R. Xu, J. Pei, Y. W. Myint, F. Wang, Z. Wang, S. Zhang, Z. Yu, and Y. Lu, Light Sci. Appl. 4, e312 (2015).

${ }^{144}$ Z. Zhang, J. Xie, D. Yang, Y. Wang, M. Si, and D. Xue, Appl. Phys. Express 8, 055201 (2015).

${ }^{145}$ S. Y. Ma, P. Zhou, L. Z. Sun, and K. W. Zhang, Phys. Chem. Chem. Phys. 18, 8723 (2016).

${ }^{146}$ Z. Zhu, J. Guan, and D. Tomanek, Phys. Rev. B 91, 161404 (2015).

${ }^{147}$ L. Kou, Y. Ma, X. Tan, T. Frauenheim, A. Du, and S. Smith, J. Phys. Chem. C 119, 6918 (2015).

${ }^{148}$ K. Luo, S. Y. Chen, and C. G. Duan, Sci. China Phys. Mech. Astron. 58, 87301 (2015).

${ }^{149}$ Y. Wang and Y. Ding, J. Phys: Condens. Matter. 27, 225304 (2015),

${ }^{150}$ H. Zhang, Y. Ma, and Z. Chen, Nanoscale 7, 19152 (2015).

${ }^{151}$ C. Wang, Q. Xia, Y. Nie, M. Rahman, and G. Guo, AIP Adv. 6, 035204 (2016).

${ }^{152}$ M. Pumera and Z. Sofer, Adv. Mater. 29(21), 1605299 (2017).

${ }^{153}$ F. Xia, S. Xiong, Y. He, Z. Shao, X. Zhang, and J. Jie, J. Phys. Chem. C 121(35), 19530 (2017).

${ }^{154}$ H. Shu, Y. Li, X. Niu, and J. Guo, J. Mater. Chem. C 6(1), 83 (2018).

${ }^{155}$ Y. Wang, P. Huang, M. Ye, R. Quhe, Y. Pan, H. Zhang, H. Zhong, J. Shi, and J. Lu, Chem. Mater. 29(5), 2191 (2017).

${ }^{156}$ Y. Xu, B. Peng, H. Zhang, H. Shao, R. Zhang, and H. Zhu, Ann. Phys. 529(4), 1600152 (2017).

${ }^{157}$ S. Zhang, Z. Yan, Y. Li, Z. Chen, and H. Zeng, Angew. Chem. Int. Ed. 54(10), 3112 (2015).

${ }^{158}$ D. Singh, S. K. Gupta, Y. Sonvane, and S. Sahoo, Nanotechnology 28(49), 495202 (2017).

${ }^{159}$ D. Kecik, E. Durgun, and S. Ciraci, Phys. Rev. B 94, 205410 (2016).

${ }^{160} \mathrm{M}$. Buscema, D. J. Groenendijk, S. I. Blanter, G. A. Steele, H. S. J. van der Zant, and A. Castellanos-Gomez, Nano Lett. 14, 3347 (2014).

${ }^{161}$ V. Tran, R. Soklaski, Y. Liang, and L. Yang, Phys. Rev. B 89, 235319 (2014).

${ }^{162}$ T. C. Berkelbach, M. S. Hybertsen, and D. R. Reichman, Phys. Rev. B 88, 045318 (2013).

${ }^{163}$ M. M. Ugeda, A. J. Bradley, S. F. Shi, F. H. da Jornada, Y. Zhang, D. Y. Qiu, W. Ruan, S. K. Mo, Z. Hussain, Z. X. Shen, F. Wang, S. G. Louie, and M. F. Crommie, Nat. Mater. 13, 1091 (2014).

${ }^{164}$ Z. Ye, T. Cao, K. O’Brien, H. Zhu, X. Yin, Y. Wang, S. G. Louie, and X. Zhang, Nature 513, 214 (2014).

${ }^{165}$ K. He, N. Kumar, L. Zhao, Z. Wang, K. F. Mak, H. Zhao, and J. Shan, Phys. Rev. Lett. 113, 026803 (2014).

${ }^{166}$ D. Cakir, C. Sevik, and F. M. Peeters, Phys. Rev. B 92, 165406 (2015).

${ }^{167}$ H. P. Komsa and A. V. Krasheninnikov, Phys. Rev. B 86, 241201(R) (2012).

${ }^{168}$ A. Chaves, M. Z. Mayers, F. M. Peeters, and D. R. Reichman, Phys. Rev. B 93 , 115314 (2016).

${ }^{169}$ J. Lee, W. C. Tian, W. L. Wang, and D. X. Yao, Sci. Rep. 5, 11512 (2015).

${ }^{170}$ G. Wang, R. Pandey, and S. P. Karna, ACS Appl. Mater. Interfaces 7, 11490 (2015).

${ }^{171}$ D. Singh, S. K. Gupta, S. Y. Sonvane, and I. Lukacevic, J. Mater. Chem. C 4, $6386(2016)$.
${ }^{172}$ S. Zhang, M. Xie, F. Li, Z. Yan, Y. Li, E. Kan, W. Liu, Z. Chen, and H. Zeng, Angew. Chem. Int. Ed. 55, 1666 (2016).

${ }^{173}$ Y. Xu, B. Peng, H. Zhang, H. Shao, R. Zhang, and H. Zhu, Ann. Phys. 529, 1600152 (2017).

${ }^{174}$ H. Shu, Y. Li, X. Niu, and J. Guo, J. Mater. Chem. C 6, 83 (2018).

${ }^{175}$ Y. Luo, Y. Li, P. Guo, W. Chen, Y. Tang, and Y. Jia, Mod. Phys. Lett. B 31, 1750341 (2017).

${ }^{176}$ Y. Wang and Y. Ding, Nanoscale Res. Lett. 10, 254 (2015).

${ }^{177}$ S. Singh and A. H. Romero, Phys. Rev B 95, 165444 (2017).

${ }^{178}$ D. Kecik, V. O. Ozcelik, E. Durgun, and S. Ciraci, Phys. Chem. Chem. Phys. 21, 7907 (2019).

${ }^{179}$ D. R. Kripalani, A. A. Kistanov, Y. Cai, M. Xue, and K. Zhou, Phys. Rev. B 98, 085410 (2018).

${ }^{180}$ S. Zhang, S. Guo, Z. Chen, Y. Wang, H. Gao, J. G. Herrero, P. Ares, F. Zamora, Z. Zhuf, and H. Zeng, Chem. Soc. Rev. 47, 982 (2018).

${ }^{181}$ F. Chu, M. Chen, Y. Wang, Y. Xie, B. Liu, Y. Yang, X. An, and Y. Zhang, J. Mater. Chem. C 6, 2509 (2018).

${ }^{182} \mathrm{G}$. Wang, S. Higgins, K. Wang, D. Bennett, N. Milosavljevic, J. J. Magan, S. Zhang, X. Zhang, J. Wang, and W. J. Blau, Appl. Opt. 57, E147 (2018).

${ }^{183}$ Y. Xu, B. Peng, H. Zhang, H. Shao, R. Zhang, and H. Zhu, Ann. Phys. 529(4), 1 (2017).

${ }^{184}$ T. Nagao, J. T. Sadowski, M. Saito, S. Yaginuma, Y. Fujikawa, T. Kogure, T. Ohno, Y. Hasegawa, S. Hasegawa, and T. Sakurai, Phys. Rev. Lett. 93, 105501 (2004).

${ }^{185}$ Yu. M. Koroteev, G. Bihlmayer, E. V. Chulkov, and S. Blügel, Phys. Rev. B 77, 045428 (2008).

${ }^{186}$ S. Yaginuma, K. Nagaoka, T. Nagao, G. Bihlmayer, Y. M. Koroteev, E. V. Chulkov, and T. Nakayama, J. Phys. Soc. Jpn. 77, 014701 (2008).

${ }^{187}$ M. Wada, S. Murakami, F. Freimuth, and G. Bihlmayer, Phys. Rev. B 83, 121310 (2011).

${ }^{188}$ Z. Liu, C. X. Liu, Y. S. Wu, W. H. Duan, F. Liu, and J. Wu, Phys. Rev. Lett. 107, 136805 (2011).

${ }^{189}$ F. Yang, L. Miao, Z. F. Wang, M. Y. Yao, F. Zhu, Y. R. Song, M. X. Wang, J. P. Xu, A. V. Fedorov, Z. Sun, G. B. Zhang, C. Liu, F. Liu, D. Qian, C. L. Gao, and J. F. Jia, Phys. Rev. Lett. 109, 016801 (2012).

${ }^{190}$ L. Chen, Z. F. Wang, and F. Liu, Phys. Rev. B 87, 235420 (2013).

${ }^{191}$ L. Cheng, H. Liu, X. Tan, J. Zhang, J. Wei, H. Lv, J. Shi, and X. Tang, J. Phys. Chem. C 118, 904 (2014).

${ }^{192}$ C. H. Hsu, Z. Q. Huang, F. C. Chuang, C. C. Kuo, Y. T. Liu, H. Lin, and A. Bansil, New J. Phys. 17, 025005 (2015).

${ }^{193}$ R. R. Q. Freitas, R. Rivelino, F. de Brito Mota, and C. M. C. de Castilho, J. Phys. Chem. C 119, 23599 (2015).

${ }^{194}$ Y. M. Koroteev, G. Bihlmayer, E. V. Chulkov, and S. Blügel, Phys. Rev. B 77, 045428 (2008).

${ }^{195}$ R. B. Chen, D. J. Jang, M. C. Lin, and M. F. Lin, Opt. Lett. 43, 6089 (2018).

${ }^{196}$ L. Lu, Z. Liang, L. Wu, Y. Chen, Y. Song, S. C. Dhanabalan, J. S. Ponraj, B. Dong, Y. Xiang, F. Xing, D. Fan, and H. Zhang, Laser Photonics Rev. 12, 1700221 (2018).

${ }^{197}$ H. Huang, X. Ren, Z. Li, H. Wang, Z. Huang, H. Qiao et al., Nanotechnology 29, 235201 (2018).

${ }^{198}$ W. Z. Xiao, G. Xiao, Q. Y. Rong, and L. L. Wang, Mater. Res. Express 5, 035903 (2018).

${ }^{199}$ S. B. Pillai, S. D. Dabhi, S. Narayan, and P. K. Jha, AIP Conf. Proc. 1942(1), 090022 (2018).

${ }^{200}$ W. X. Ji, C. W. Zhang, M. Ding, P. Li, and P. J. Wang, J. Mater. Chem. C 5, 2649 (2017).

${ }^{201}$ S. Singh, I. Valencia-Jaime, O. Pavlic, and A. H. Romero, Phys. Rev. B 97, 054108 (2018).

${ }^{202}$ C. G. Tan, P. Zhou, J. G. Lin, and L. Z. Sun, Phys. Status. Solidi-RRL 11, 1700051 (2017).

${ }^{203}$ T. Yildirim and S. Ciraci, Phys. Rev. Lett. 94(17), 175501 (2005).

${ }^{204}$ E. Durgun, S. Ciraci, and T. Yildirim, Phys. Rev. B 77, 085405 (2008).

${ }^{205}$ C. Ataca, E. Aktürk, S. Ciraci, and H. Ustunel, Appl. Phys. Lett. 93(4), 043123 (2008).

${ }^{206}$ H. Sevinçli, M. Topsakal, E. Durgun, and S. Ciraci, Phys. Rev. B 77, 195434 (2008). 
${ }^{207}$ Y. Kadioglu, O. Üzengi Aktürk, E. Aktürk, and S. Ciraci, J. Phys. Chem. C 121, 6329 (2017).

${ }^{208}$ V. V. Kulish, O. I. Malyi, C. Persson, and P. Wu, Phys. Chem. Chem. Phys. 17, 992 (2015).

${ }^{209}$ M. Sun, W. Tang, Q. Ren, S. K. Wang, J. Yu, and Y. Du, Appl. Surf. Sci. 356, 110 (2015)

${ }^{210}$ M. Sun, Y. Hao, Q. Ren, Y. Zhao, Y. Du, and W. Tang, Solid State Commun. 242, 36 (2016).

${ }^{211}$ P. Srivastava, K. P. S. S. Hembram, H. Mizuseki, K. R. Lee, S. S. Han, and S. Kim, J. Phys. Chem. C 119, 6530 (2015).

${ }^{212}$ W. Yu, Z. Zhu, C. Y. Niu, C. Li, J. H. Cho, and Y. Jia, Nanoscale Res. Lett. 11, 77 (2016).

${ }^{213}$ S. Chintalapati, L. Shen, Q. Xiong, and Y. P. Feng, Appl. Phys. Lett. 107, 072401 (2015).

${ }^{214}$ Z. D. Sha, Q. X. Pei, Y. Y. Zhang, and Y. W. Zhang, Nanotechnology 27, 315704 (2016)

${ }^{215}$ A. Kistanov, Y. Cai, K. Zhou, S. V. Dmitriev, and Y. W. Zhang, 2D Mater. 4, 015010 (2017)

${ }^{216}$ Y. Cai, Q. Ke, G. Zhang, and Y. W. Zhang, J. Phys. Chem. C 119, 3102 (2015).

${ }^{217}$ A. Ziletti, A. Carvalho, D. K. Campbell, D. F. Coker, and A. H. Castro Neto, Phys. Rev. Lett. 114, 046801 (2015).

${ }^{218}$ G. Wang, W. J. Slough, R. Pandey, and S. P. Karna, 2D Mater. 3, 025011 (2016).

${ }^{219}$ F. Ding and B. I. Yakobson, Front. Phys. 6, 142 (2011).

${ }^{220}$ K. M. Fair, X. Y. Cui, L. Li, C. C. Shieh, R. K. Zheng, Z. W. Liu, B. Delley, M. J. Ford, S. P. Ringer, and C. Stampfl, Phys. Rev. B 87, 014102 (2013).

${ }^{221}$ A. Kuang, M. Kuang, H. Yuan, G. Wang, H. Chen, and X. Yang, Appl. Surf. Sci. 410, 505 (2017)

${ }^{222}$ X. B. Li, P. Guo, T. F. Cao, H. Liu, W. M. Lau, and L. M. Liu, Sci. Rep. 5, 10848 (2015).

${ }^{223}$ L. J. Kong, G. H. Liu, and Y. J. Zhang, RSC Adv. 6, 10919 (2016).

${ }^{224}$ M. Y. Liu, Y. Huang, Q. Y. Chen, C. Cao, and Y. He, Sci. Rep. 6, 29114 (2016).

${ }^{225}$ X. P. Chen, L. M. Wang, X. Sun, R. S. Meng, J. Xiao, H. Y. Ye, and G. Q. Zhang, IEEE Electron Dev. Lett. 38, 661 (2017).

${ }^{226}$ Md. S. Khan, A. Srivastava, and R. Pandey, RSC Adv. 6, 72634 (2016).

${ }^{227}$ M. Liu, Q. Chen, Y. Huang, C. Cao, and Y. He, Superlattices and Microstructures 100, 131 (2016).

${ }^{228}$ M. Sun, S. Wang, Y. Du, J. Yu, and W. Tang, Appl. Surf. Sci. 389, 594 (2016).

${ }^{229}$ M. Luo, Y. H. Shen, and T. L. Yin, JETP Lett. 104(8), 557 (2016).

${ }^{230}$ X. Liu, L. Liu, L. Yang, X. Wu, and P. Chu, J. Phys. Chem. C 120, 24917 (2016).

${ }^{231} \mathrm{~K}$. Iordanidou, J. Kioseoglou, V. V. Afanasev, A. Stesmans, and M. Houssa, Phys. Chem. Chem. Phys. 19, 9862 (2017)

${ }^{232}$ M. Mushtag, Y. Zhou, and X. Xiang, J. Phys.: Condens. Matter 30, 195305 (2018).

${ }^{233}$ O. Üzengi Aktürk, E. Aktürk, and S. Ciraci, Phys. Rev. B 93, 035450 (2016).

${ }^{234}$ R. S. Meng, M. Cai, J. K. Jiang, Q. H. Liang, X. Sun, Q. Yang, C. J. Tan, and X. P. Chen, IEEE Electron Dev. Lett. 38, 134 (2017).

${ }^{235}$ A. A. Kistanov, Y. Cai, D. R. Kripalani, K. Zhou, S. V. Dmitriev, and Y. W. Zhang, J. Mater. Chem. C 6, 4308 (2018).

${ }^{236}$ T. T. Li, C. He, and W. X. Zhang, Appl. Surf. Sci. 427, 388 (2018).

${ }^{237}$ M. Xie, S. Zhang, B. Cai, Y. Zou, and H. Zeng, RSC Adv. 6, 14620 (2016).

${ }^{238}$ L. Yang, Y. Song, W. Mi, and X. Wang, RSC Adv. 6, 66140 (2016).

${ }^{239}$ X. Sun, Y. Liu, Z. Song, Y. Li, W. Wang, H. Lin, L. Wang, and Y. Li, J. Mater. Chem. C 5, 4159 (2017).

${ }^{240}$ Y. Ding and Y. Wang, J. Phys. Chem. C 119, 10610 (2015).

${ }^{241}$ P. Rastogi, S. Kumar, S. Bhowmick, A. Agarwal, and Y. S. Chauhan, IETE J. Res. 63, 205 (2017).

${ }^{242}$ H. Zhu, C. Zhou, X. Wang, X. Chen, W. Yang, Y. Wu, and W. Lin, Phys. Status Solidi B 253, 1156 (2016).

${ }^{243}$ J. Qiao, X. Kong, Z.-X. Hu, F. Yang, and W. Ji, Nat. Commun. 5, 4475 (2014).

${ }^{244}$ D. W. Boukhvalov, A. N. Rudenko, D. A. Prishchenko, V. G. Mazurenko, and M. I. Katsnelson, Phys. Chem. Chem. Phys. 17, 15209 (2015).

${ }^{245}$ S. Nahas, B. Ghosh, S. Bhowmick, and A. Agarwal, Phys. Rev. B 93, 165413 (2016).

${ }^{246}$ Y. Li and X. Chen, 2D Mater. 1, 031002 (2014).

${ }^{247}$ S. Zhang, Y. Hu, Z. Hu, B. Cai, and H. Zeng, Appl. Phys. Lett. 107, 022102 (2015).
${ }^{248}$ Y. P. Wang, W. X. Ji, C. W. Zhang, P. Li, F. Li, M. J. Ren, X. L. Chen, M. Yuan, and P. J. Wang, Sci. Rep. 6, 20342 (2016).

${ }^{249}$ W. Tang, M. Sun, Q. Ren, S. Wang, and J. Yu, Appl. Surf. Sci. 376, 286 (2016).

${ }^{250}$ D. Wang, L. Chen, C. Shi, X. Wang, G. Cui, P. Zhang, and Y. Chen, Sci. Rep 6, 28487 (2016).

${ }^{251}$ J. Zhao, W. Guo, and J. Ma, Nano Res. 10(2), 491 (2017).

${ }^{252}$ M. Y. Liu, Z. Y. Li, Q. Y. Chen, Y. Huang, C. Cao, and Y. He, Sci. Rep. 7, 4773 (2017).

${ }^{253}$ D. Wang, L. Chen, C. Shi, X. Wang, G. Cui, P. Zhang, and Y. Chen, New J Phys. 18, 033026 (2016)

${ }^{254}$ Z. Song, C. C. Liu, J. Yang, J. Han, M. Ye, B. Fu, Y. Yang, Q. Niu, J. Lu, and Y. Yao, NPG Asia Mater. 6, e147 (2014).

${ }^{255}$ T. Zhang, Y. Y. Qi, X. R. Chen, and L. C. Cai, Phys. Chem. Chem. Phys. 18, 30061 (2016).

${ }^{256}$ K. H. Yeoh, T. L. Yoon, D. S. Ong, T. L. Lim, and Y. Z. Abdullahi, Phys. Chem. Chem. Phys. 19, 25786 (2017).

${ }^{257}$ S. Zhang, W. Zhou, Y. Ma, J. Ji, B. Cai, S. A. Yang, Z. Zhu, Z. Chen, and H. Zeng, Nano Lett. 17, 3434 (2017).

${ }^{258}$ J. Zhang, H. J. Liu, L. Cheng, J. Wei, J. H. Liang, D. D. Fan, J. Shi, X. F. Tang, and Q. J. Zhang, Sci. Rep. 4, 6452 (2014).

${ }^{259}$ H. Guo, N. Lu, J. Dai, X. Wu, and X. C. Zeng, J. Phys. Chem. C 118, 14051 (2014).

${ }^{260}$ A. Carvalho, A. S. Rodin, and A. H. Castro Neto, EPL 108, 47005 (2014).

${ }^{261}$ Q. Wu, L. Shen, M. Yang, Y. Cai, Z. Huang, and Y. P. Feng, Phys. Rev. B 92, 035436 (2015).

${ }^{262}$ R. Swaroop, P. K. Ahluwalia, K. Tankeshwar, and A. Kumar, RSC Adv. 7, 2992 (2017).

${ }^{263}$ H. Sarvari, C. Liu, A. H. Ghayour, P. Shenavar, Z. Chen, and R. Ghayour, Phys. E 91, 161 (2017).

${ }^{264}$ B. Zhou, B. Zhou, P. Liu, and G. Zhou, Phys. Lett. A 382, 193 (2018).

${ }^{265}$ H. S. Tsai, C. W. Chen, C. H. Hsiao, H. Ouyang, and J. H. Liang, Chem. Commun. 52, 8409 (2016).

${ }^{266}$ Y. Luo, Y. Li, P. Guo, W. Chen, Y. Tang, and Y. Jia, Mod. Phys. Lett. B 31(36), 1750341 (2017).

${ }^{267}$ Y. Wang and Y. Ding, Nanoscale Res. Lett. 10, 254 (2015).

${ }^{268}$ V. Nagarajan and R. Chandiramouli, Phys. E 97, 98 (2018).

${ }^{269}$ Y. Song, X. Wang, and W. Mi, NPJ Quantum Mater. 2, 15 (2017).

${ }^{270} \mathrm{X}$. Wu, X. Zhang, X. Wang, and Z. Zeng, AIP Adv. 6, 045318 (2016).

${ }^{271}$ K. W. Yang, M. J. Li, X. J. Zhang, X. M. Li, Y. L. Gao, and M. Q. Long, Chin. Phys. B 26, 098509 (2017).

${ }^{272}$ S. Wang, X. Zhang, Y. Huang, and C. Sun, Phys. Chem. Chem. Phys. 20, 25716 (2018)

${ }^{273}$ M. Abid, A. Shoaib, M. H. Farooq, H. Wu, D. Ma, and B. Fu, J. Phys. Chem. Solids 110, 167 (2017).

${ }^{274}$ W. Ning, F. Kong, J. Hu, Y. Han, J. Yang, H. Du, Y. Zhang, and M. Tian, Appl. Phys. Lett. 110, 123101 (2017)

${ }^{275}$ W. Ning, F. Kong, Y. Han, H. Du, J. Yang, M. Tian, and Y. Zhang, Sci. Rep. 4, 7086 (2014)

${ }^{276}$ W. Ning, F. Kong, C. Xi, D. Graf, H. Du, Y. Han, J. Yang, K. Yang, M. Tian, and Y. Zhang, ACS Nano 8(7), 7506 (2014).

${ }^{277}$ X. Wang, C. Xu, H. Hu, P. Wang, G. Bian, W. Tan, S. A. Brown, and T. C. Chiang, EPL 119, 27002 (2017).

${ }^{278}$ J. S. Li, W. L. Wang, and D. X. Yao, Sci. Rep. 6, 34177 (2016).

${ }^{279}$ A. N. Rudenko and M. I. Katsnelson, Phys. Rev. B 89, 201408(R) (2014).

${ }^{280}$ L. Li, Y. Yu, G. J. Ye, Q. Ge, X. Ou, H. Wu, D. Feng, X. H. Chen, and Y. Zhang, Nat. Nanotechnol. 9, 372 (2014).

${ }^{281}$ T. Low, A. S. Rodin, A. Carvalho, Y. Jiang, H. Wang, F. Xia, and A. H. C. Neto, Phys. Rev. B 90, 075434 (2014).

${ }^{282}$ J. Dai and X. C. Zeng, J. Phys. Chem. Lett. 5, 1289 (2014).

${ }^{283}$ T. Zhang, J. H. Lin, Y. M. Yu, X. R. Chen, and W. M. Liu, Sci. Rep. 5, 13927 (2015).

${ }^{284}$ Z. Zhang, J. Xie, D. Yang, Y. Wang, M. Si, and D. Xue, Appl. Phys. Express 8, 055201 (2015).

${ }^{285}$ K. Luo, S. Y. Chen, and C. G. Duan, Sci. China Phys. Mech. Astron. 58, 87301 (2015).

${ }^{286}$ Y. Kadioglu, J. A. Santana, H. D. Ozaydin, F. Ersan, O. Uzengi Akturk, E. Akturk, and F. A. Reboredo, J. Chem. Phys. 148, 214706 (2018). 
${ }^{287}$ K. Mi, J. Xie, M. S. SI, and C. X. Gao, EPL 117, 27002 (2017).

${ }^{288}$ C. Gibaja, D. R. S. Miguel, P. Ares, J. G. Herrero, M. Varela, R. Gillen, J. Maultzsch, F. Hauke, A. Hirsch, G. Abellan, and F. Zamora, Angew. Chem, Int. Ed. 55, 14345 (2016).

${ }^{289}$ X. Wang, G. Bian, C. Xu, P. Wang, H. Hu, W. Zhou, S. A. Brown, and T.-C. Chiang, Nanotechnology 28, 395706 (2017).

${ }^{290}$ M. Xie, S. Zhang, B. Cai, Y. Gu, X. Liu, E. Kan, and H. Zeng, Nano Energy 38, 561 (2017).

${ }^{291}$ L. Lu, W. Wang, L. Wu, X. Jiang, Y. Xiang, J. Li, D. Fan, and H. Zhang, ACS Photonics 4, 2852 (2017).

${ }^{292}$ L. Shulenburger, A. D. Baczewski, Z. Zhu, J. Guan, and D. Tománek, Nano Lett. 15, 8170 (2015).

${ }^{293}$ Y. X. Deng, Z. Luo, N. J. Conrad, H. Liu, Y. J. Gong, S. Najmaei, P. M. Ajayan, J. Lou, X. F. Xu, and P. D. Ye, ACS Nano 8, 8292 (2014).

${ }^{294}$ K. Gong, L. Zhang, W. Ji, and H. Guo, Phys. Rev. B 90, 125441 (2014).

${ }^{295}$ D. A. Ospina, E. Cisternas, C. A. Duque, and J. D. Correa, Surf. Sci. 669, 95 (2018).

${ }^{296}$ J. E. Padilha, A. Fazzio, and A. J. R. da Silva, Phys. Rev. Lett. 114, 066803 (2015).

${ }^{297}$ W. Hu, T. Wang, and J. Yang, J. Mater. Chem. C 3, 4756 (2015).

${ }^{298}$ A. R. Davletshin, S. V. Ustiuzhanina, A. A. Kistanov, D. Saadatmand, S. V. Dmitriev, K. Zhou, and E. A. Korznikova, Phys. B 534, 63 (2018).

${ }^{299}$ X. Wang, X. Yang, B. Wang, G. Wang, and J. Wan, Carbon 130, 120 (2018).

${ }^{300}$ N. Gillgren, D. Wickramaratne, Y. Shi, T. Espiritu, J. Yang, J. Hu, J. Wei, X. Liu, Z. Mao, K. Watanabe, T. Taniguchi, M. Bockrath, Y. Barlas, R. K. Lake, and C. N. Lau, 2D Mater. 2, 011001 (2015).

${ }^{301}$ J. E. Padilha, A. Fazzio, and A. J. R. da Silva, Phys. Rev. Lett. 114, 066803 (2015).

${ }^{302}$ V. D. S. O. Ganesan, J. Linghu, C. Zhang, Y. P. Feng, and L. Shen, Appl. Phys. Lett. 108, 122105 (2016).

${ }^{303}$ Q. Peng, Z. Wang, B. Sa, B. Wu, and Z. Sun, ACS Appl. Mater. Interfaces 8(21), 13449 (2016).

${ }^{304}$ J. Zeng, P. Cui, and Z. Zhang, Phys. Rev. Lett. 118, 046101 (2017).

${ }^{305}$ Y. Song, D. Li, W. Mi, X. Wang, and Y. Cheng, J. Phys. Chem. C 120, 5613 (2016).

${ }^{306}$ Y. Wang, M. Ye, M. Weng, J. Li, X. Zhang, H. Zhang, Y. Guo, Y. Pan, L. Xiao, J. Liu, F. Pan, and J. Lu, ACS Appl. Mater. Interfaces 9, 29273 (2017).

307.J. Su, L. Feng, and Z. Liu, RSC Adv. 6, 59633 (2016).

${ }^{308}$ H. Shu, Y. Tong, and J. Guo, Phys. Chem. Chem. Phys. 19, 10644 (2017).

${ }^{309}$ C. Xia, B. Xue, T. Wang, Y. Peng, and Y. Jia, Appl. Phys. Lett. 107, 193107 (2015).

${ }^{310}$ W. Li, T. X. Wang, X. Q. Dai, X. L. Wang, Y. Q. Ma, S. S. Chang, and Y. N. Tang, Phys. E 88, 6 (2017).

${ }^{311}$ H. Shu, Y. Tong, and J. Guo, Phys. Chem. Chem. Phys. 19, 10644 (2017).

${ }^{312}$ X. H. Li, B. J. Wang, X. L. Cai, L. W. Zhang, G. D. Wang, and S. H. Ke, RSC Adv. 7, 28393 (2017).

${ }^{313}$ X. H. Li, B. J. Wang, X. L. Cai, W. Y. Yu, L. W. Zhang, G. D. Wang, and S. H. Ke, RSC Adv. 7, 44394 (2017).

${ }^{314}$ F. Zhang, W. Li, and X. Dai, Superlattices Microstruct. 100, 826 (2016).

${ }^{315}$ W. Li, X. Wang, and X. Dai, Solid State Commun. 254, 37 (2017).

${ }^{316}$ Y. Wang and Y. Ding, Phys. Chem. Chem. Phys. 17, 27769 (2015).

${ }^{317}$ H. Liu, J. Gao, Z. Hu, and X. Shao, RSC Adv. 6, 102724 (2016).

${ }^{318} \mathrm{X}$. Chen, Q. Yang, R. Meng, J. Jiang, Q. Liang, C. Tan, and X. Sun, J. Mater. Chem. C 4, 5434 (2016).

${ }^{319}$ Y. Guo, F. Pan, M. Ye, X. Sun, Y. Wang, and J. Li, ACS Appl. Mater. Interfaces 9, 23128 (2017).

${ }^{320}$ Z. Q. Huang, F. C. Chuang, C. H. Hsu, Y. T. Liu, H. R. Chang, H. Lin, and A. Bansil, Phys. Rev. B. 88, 165301 (2013).

${ }^{321}$ H. J. Kim and J. H. Cho, Phys. Rev. B 92, 085303 (2015).

${ }^{322}$ C. G. Tan, P. Zhou, J. G. Lin, and L. Z. Sun, Phys. Status Solidi RRL 11, 1700051 (2017).

${ }^{323}$ W. Z. Xiao, G. Xiao, Q. Y. Rong, and L. L. Wang, Mater. Res. Express 5, 035903 (2018).

${ }^{324} \mathrm{H}$. Shu and J. Guo, Mater. Res. Express 5, 036302 (2018).

${ }^{325}$ D. P. Shoemaker, T. C. Chasapis, D. Do, M. C. Francisco, D. Y. Chung, S. D. Mahanti, A. Llobet, and M. G. Kanatzidis, Phys. Rev. B 87, 094201 (2013).

${ }^{326}$ Z. Zhu, J. Guan, and D. Tománek, Nano Lett. 15, 6042 (2015).
${ }^{327}$ W. Yu, C.-Y. Niu, Z. Zhu, X. Wang, and W.-B. Zhang, J. Mater. Chem. C 4, 6581 (2016).

${ }^{328}$ P. Liu, Y.-Z. Nie, Q.-L. Xia, and G.-H. Guo, Phys. Lett. A 381, 1102 (2017).

${ }^{329}$ W. Z. Xiao, G. Xiao, Q. Y. Rong, and L. L. Wang, Phys. Chem. Chem. Phys. 20, 22027 (2018).

${ }^{330}$ Q. Sun, Y. Dai, Y. Ma, N. Yin, W. Wei, L. Yu, and B. Huang, 2D Mater. 3, 035017 (2016).

${ }^{331}$ W. X. Ji, C. W. Zhang, M. Ding, P. Li, and P. J. Wang, J. Mater. Chem. C 5, 2649 (2017)

${ }^{332}$ S. Singh and A. H. Romero, Phys. Rev. B 95, 165444 (2017).

${ }^{333}$ F. Shojaei and H. S. Kang, J. Phys. Chem. C 119, 20210 (2015).

${ }^{334}$ M. Xie, S. Zhang, B. Cai, Y. Huang, Y. Zou, B. Guo, Y. Gu, and H. Zeng, Nano Energy 28, 433 (2016).

335J.-P. Tang, W.-Z. Xiao, and L.-L. Wang, Mater. Sci. Eng. B 228, 206 (2018).

${ }^{336}$ Z. H. Benam, H. Arkin, and E. Akturk, Comput. Mater. Sci. 140, 290 (2017).

${ }^{337}$ L. Zhao, W. Yi, J. Botana, F. Gu, and M. Miao, J. Phys. Chem. C 121, 28520 (2017).

${ }^{338}$ J. K. Kjerns, L. Passell, H. Taub, J. G. Dash, and A. D. Novaco, Phys. Rev. B 13, 1446 (1976).

${ }^{339}$ M. Trabelsi, J. P. Coulomb, D. Degenhardt, and H. Lauter, Surf. Sci. 377-379, 38 (1997).

${ }^{340}$ Y. Harada, M. Yamamoto, T. Baba, and T. Kita, Appl. Phys. Lett. 104, 041907 (2014).

${ }^{341}$ L. Li, Y. Yu, G. J. Ye, Q. Ge, X. Ou, H. Wu, D. Feng, X. H. Chen, and Y. Zhang, Nat. Nanotechnol. 9, 372 (2014).

${ }^{342}$ L. Kou, C. Chen, and S. C. Smith, J. Phys. Chem. Lett. 6, 2794 (2015).

${ }^{343}$ P. Yasaei, B. Kumar, T. Foroozan, C. Wang, M. Asadi, D. Tuschel, J. E. Indacochea, R. F. Klie, and A. Salehi-Khojin, Adv. Mater. 27, 1887 (2015).

${ }^{344}$ J. R. Brent, N. Savjani, E. A. Lewis, S. J. Haigh, D. J. Lewis, and P. OBrien, Chem. Commun. 50, 13338 (2014).

${ }^{345}$ H. Kaur, S. Yadav, A. K. Srivastava, N. Singh, J. J. Schneider, O. P. Sinha, V. V. Agrawal, and R. Srivastava, Sci. Rep. 6, 34095 (2016).

${ }^{346}$ A. C. Gomez, L. Vicarelli, E. Prada, J. O. Island, K. L. N. Acharya, S. I. Blanter, D. J. Groenendijk, M. Buscema, G. A. Steele, J. V. Alvarez, H. W. Zandbergen, J. J. Palacios, and H. S. J. van der Zant, 2D Mater. 1, 025001 (2014).

${ }^{347}$ M. Pumera, Trends Anal. Chem. 93, 1-6 (2017).

${ }^{348}$ W. Wen, Y. Song, X. Yan, C. Zhu, D. Du, S. Wang, A. M. Asiri, and Y. Lin, Mater. Today 21, 164 (2018).

${ }^{349}$ J. Kim, S. Sando, and T. Cui, in ASME 2017 International Mechanical Engineering Congress and Exposition(2017).

${ }^{350}$ X. Li, X. Niu, W. Zhao, W. Chen, C. Yin, Y. Men, G. Li, and W. Sun, Electrochem. Commun. 86, 68 (2018).

${ }^{351}$ O. Osters, T. Nilges, F. Bachhuber, F. Pielnhofer, R. Weihrich, M. Schçneich, and P. Schmidt, Angew. Chem., Int. Ed. 51, 2994 (2012).

${ }^{352}$ B. Liu, M. Köpf, A. N. Abbas, X. Wang, Q. Guo, Y. Jia, F. Xia, R. Weihrich, F. Bachhuber, F. Pielnhofer, H. Wang, R. Dhall, S. B. Cronin, M. Ge, X. Fang, T. Nilges, and C. Zhou, Adv. Mater. 27, 4423 (2015).

${ }^{353}$ J. Shah, W. Wang, H. M. Sohail, and R. I. G. Uhrberg, preprint arXiv:1812.05978 (2018).

${ }^{354}$ T. Lei, C. Liu, J. L. Zhao, J. M. Li, Y. P. Li, J. O. Wang, R. Wu, H. J. Qian, H. Q. Wang, and K. Ibrahim, J. Appl. Phys. 119, 015302 (2016).

${ }^{355}$ J. Ji, X. Song, J. Liu, Z. Yan, C. Huo, S. Zhang, M. Su, L. Liao, W. Wang, Z. Ni, Y. Hao, and H. Zeng, Nat. Commun. 7, 13352 (2016).

${ }^{356}$ Y. Song, Z. Liang, X. Jiang, Y. Chen, Z. Li, L. Lu, Y. Ge, K. Wang, J. Zheng, S. Lu, J. Ji, and H. Zhang, 2D Mater. 4, 045010 (2017).

${ }^{357}$ P. Ares, F. Zamora, and J. G. Herrero, ACS Photonics 4, 600 (2017).

${ }^{358}$ L. Lu, X. Tang, R. Cao, L. Wu, Z. Li, G. Jing, B. Dong, S. Lu, Y. Li, Y. Xiang, J. Li, D. Fan, and H. Zhang, Adv. Opt. Mater. 5, 1700301 (2017).

${ }^{359}$ M. F. Deschênes, O. Waller, T. O. Menteş, A. Locatelli, S. Mukherjee, F. Genuzio, P. L. Levesque, A. Hébert, R. Martel, and O. Moutanabbir, Nano Lett. 17, 4970 (2017).

${ }^{360}$ T. Märkl, P. J. Kowalczyk, M. L. Ster, I. V. Mahajan, H. Pirie, Z. Ahmed, G. Bian, X. Wang, T. C. Chiang, and S. A. Brown, 2D Mater. 5, 011002 (2018). 
${ }^{361}$ G. Zhang, X. Tang, X. Fu, H. Zhang, Q. Liu, M. Gong, W. Chen, and B. Shabbir, Nanoscale 11, 1762 (2019).

${ }^{362}$ Y. Lu, W. Xu, M. Zeng, G. Yao, L. Shen, M. Yang, Z. Luo, F. Pan, K. Wu, T. Das, P. He, J. Jiang, J. Martin, Y. P. Feng, H. Lin, and X. S. Wang, Nano Lett. 15, 80 (2015).

${ }^{363}$ I. Kokubo, Y. Yoshiike, K. Nakatsuji, and H. Hirayama, Phys. Rev. B 91, 075429 (2015).

${ }^{364}$ K. Ishioka and M. Kitajima, Phys. Rev. B 91, 125431 (2015).

${ }^{365}$ N. Kawakami, C. L. Lin, M. Kawai, R. Arafune, and N. Takagi, Appl. Phys. Lett. 107, 031602 (2015).
${ }^{366}$ E. S. Walker, S. R. Na, D. Jung, S. D. March, J. S. Kim, T. Trivedi, W. Li, L. Tao, M. L. Lee, K. M. Liechti, D. Akinwande, and S. R. Bank, Nano Lett. 16, 6931 (2016).

${ }^{367}$ F. Reis, G. Li, L. Dudy, M. Bauernfeind, S. Glass, W. Hanke, R. Thomale, J. Schäfer, and R. Claessen, Science 357(6348), 287 (2017).

${ }^{368}$ L. Lu, Z. Liang, L. Wu, Y. Chen, Y. Song, S. C. Dhanabalan, J. S. Ponraj, B. Dong, Y. Xiang, F. Xing, D. Fan, and H. Zhang, Laser Photonics Rev. 12, 1700221 (2018).

${ }^{369}$ R. Devasenathipathy, R. Karthik, S. M. Chen, V. Mani, V. S. Vasantha, M. A. Ali, M. S. Elshikh, B. S. Lou, and F. M. A. Al-Hemaid, Electroanalysis 27, 2341 (2015). 